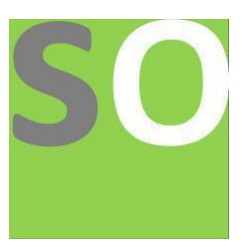

Article title: Causality in physical processes: Planck's quantum of action and universal nonequilibrium dynamics Authors: Shariq Azhar[1]

Affiliations: Independent Researcher[1]

Orcid ids: 0000-0003-3612-3849[1]

Contact e-mail: sa2001@nyu.edu

License information: This work has been published open access under Creative Commons Attribution License $\mathrm{http}: / / c r e a t i v e c o m m o n s . o r g / l i c e n s e s / b y / 4.0 /$, which permits unrestricted use, distribution, and reproduction in any medium, provided the original work is properly cited. Conditions, terms of use and publishing policy can be found at https://www.scienceopen.com/.

Preprint statement: This article is a preprint and has not been peer-reviewed, under consideration and submitted to ScienceOpen Preprints for open peer review.

Links to data: https://osf.io/fcx8t/files

DOI: 10.14293/S2199-1006.1.SOR-.PPPYS4V.v1

Preprint first posted online: 14 June 2021

Keywords: Planck's constant, cellular automata, gravitation, Coulomb force, nuclear force, relational causality, quantum mechanics, atomic spectra, nonlinear dynamics, fine-structure constant 


\title{
Causality in physical processes: Planck's quantum of action and universal nonequilibrium dynamics
}

\author{
Shariq Azhar*
}

\begin{abstract}
A mathematical model is presented that describes how the diverse phenomena in nature can arise from a common foundation rooted in an objective framework of relational causality. Key equations of conventional physical theories are derived anew. Rigorous derivations are shown for such fundamental relationships as the Schrödinger equation, Bohr's formula for the hydrogen spectrum, Newton's gravitational law, Coulomb's electrostatic law, Compton shift formula, and other principal equations of electromagnetism, atomic theory, optics, and thermodynamics. Nuclear forces that bind nucleons and pry them apart are shown to arise from a single universal electrostatic force that also gives rise to the Coulomb force. Dark matter is shown to be an ever-present material content that subsists in the fabric of space everywhere. The precise value of Coulomb's constant is derived on purely theoretical grounds, while hitherto unknown values are predicted for the electric dipole moments of electrons and protons. The first of three components of the core hypothesis of this model is that there exists a primordial seed particle of which all that lies in the universe is ultimately composed, and that it is defined by Planck's constant. Space is modeled as a lattice of contiguous cells that are composed entirely of these particles, and are inherently elastic. The second component is that the universe evolves temporally through a recursive process, emulating a system of cellular automata. The third component stipulates that any compression produced in a cell by its ambient conditions generates potential energy according to classical elasticity. Thus, every cell seeks to minimize its stored potential energy at each step in its temporal evolution, in pursuit of elastic equilibrium. This core hypothesis in respect to the physical character of space, time, and energy is shown to lead logically to a universal dynamic that gives rise to the physical effects observed in nature at every scale.
\end{abstract}

Keywords: Planck's constant, cellular automata, gravitation, Coulomb force, nuclear force, relational causality

\section{INTRODUCTION}

In the four centuries since the scientific revolution, the scope of physical theories has grown to encompass every notable facet of nature. However, because these advances have occurred through a largely unstructured process, it is rare to find discernible links that explicitly connect the various distinct domains of physics. Few definitive relationships are evident, for instance, between the domains of electromagnetism and thermodynamics, or particle physics and optics, or quantum mechanics and gravitation. This paper aims to show that there exists a set of unifying principles through which it can be established that the diverse phenomena in nature share a common foundation rooted in a framework of relational causality.

The viability of such a model is contingent, above all, on the agreement of its predictions with scientific observations. The model must be logically self-consistent, and its arguments must be supported by sound mathematical reasoning to demonstrate their veracity credibly. Moreover, its mathematical formulations and predictions must be consistent with those of well-tested conventional theories, and it should furnish rational explanations for phenomena that existing theories fail to elucidate satisfactorily. Conversely, the viability of an alternative theoretical model does not require its foundations or logic to be in complete accordance with the domain-specific precepts of existing theories. Rather, it is to be expected that the principles underlying a model that proposes to unify the diverse domains of physics through a common source of causality would differ fundamentally from those of conventional physical theories.

The model presented herein is based on a threecomponent core hypothesis. The first component is the ontological proposition that there exist primordial particles, hereinafter called photinos, of which all of space and matter is ultimately composed. Described as the very source of inertial mass as well as energy, the photino is defined by the elementary quantum of action given by Planck's constant [1]. The proposed model further posits that photinos are distributed spatially in the form of contiguous photino packets, hereinafter called cells, where each cell is autonomous, elastic, and mobile. Space is thus modeled as a tessellated lattice of contiguous elastic cells of variable volume and photino content.

The second component of the hypothesis is that, as a system, the universe evolves synchronously in discrete time steps. Its evolution is governed by a set of 
mathematical relationships that describe the state of its constituent cells and their interactions with neighboring cells and the photinos therein. The universe thus emulates a system of cellular automata [2] in which all physical activity results from the deterministic application of a fixed set of rules at the elemental level of the cell. The rules are given as mathematical functions that determine the change in the state of each cell at each step in the temporal evolution of the system. This stipulation renders the universe inherently mechanical, where the aggregation of cell-level activity describes the behavior of the system as a whole as well as that of each subsystem therein. This model is referred to herein as photodynamics.

The proposed model draws upon a substantial body of prior work on the concept of a spatially discrete and temporally recursive universe and on the character and behavior of cellular automata. The theoretical foundations of these concepts were laid in the works of Ulam [3], von Neumann [4], Minsky [5], and Conway [6], among others. Based on his extensive study of the subject, Wolfram [7] pointed to a subliminal connection between the nonlinear complex behavior of cellular automata and physical processes in nature. 't Hooft [8] described how ambiguities associated with the Copenhagen interpretation of quantum mechanics could, in principle, be resolved by interpreting quantum mechanics within a deterministic framework of cellular automata. Wheeler [9] argued that the continuum in respect to both space and time is an idealization and that "no account of existence can ... rate as fundamental which does not translate all of continuum physics into the language of [discrete] bits." In addition, he endeavored to "understand time and space and all other features that distinguish physics ... as the ... selfgenerated organs of a self-synthesized information system."

A deep similarity indeed exists between a spatially discrete recursive universe and a system of cellular automata that evolves dynamically through an iterative process of self-synthesis. Both are mechanistic in character, where, in the present context, rule-based binary decision processes govern their spatial and temporal evolution. Moreover, cellular automata provide an efficient means of modeling the recursive behavior of a universe composed of discrete elements, in much the same sense as differential calculus enables efficient modeling of the motion of a body.

The notion of a spatially discrete recursive universe can be easily misconstrued as implying an axiomatic denial of the continuity of time and space. To be clear, continuity is an innate feature of both, because its absence carries the irrational connotation that the progression of time, or the span of a spatial separation, entails discontinuity at some level. The question therefore is not whether time and space are continuous or discrete, but rather whether discretization is logically consistent with their innate continuity. Mathematically, a continuum may be divided into any number of intervals of arbitrary size. The number of intervals is then an integer quantity, whereas the measure of each interval is a continuous quantity. Continuity thus devolves to each subdivision of the continuum. In this sense, space and time are rendered discrete in composition without implying an absence of underlying continuity. The dichotomy between discrete and continuous quantities is of fundamental importance in photodynamics. It is responsible for the mechanism that produces spatial structure and order at every scale, from the concentric symmetry of an atom to the clustering of galaxies across the cosmos. It is also an essential factor in the creation of electric charge and numerous other physical effects.

The third component of the hypothesis is that the propensities induced in physical systems by the innate elasticity of their cellular elements constitute the source of all dynamic activity in nature. As with every elastic body, a cell seeks to attain a state of elastic equilibrium such that its extant volume is equal to its equilibrium volume. A state of elastic nonequilibrium in a cell is hence described by the difference between its extant and equilibrium volumes. Such a difference exists when a cell is subjected to compression by prevailing conditions in its ambient system. Based on classical laws for elastic bodies, the displacement of a cell from elastic equilibrium generates potential energy that is proportional to the square of the displacement. The innate propensity of cells within a system to attain elastic equilibrium thus induces a compulsion in every cell to reduce its potential energy maximally at each step in the temporal evolution of the system. Because every physical system, regardless of its scale or composition, consists ultimately of contiguous cells, every physical system tends inexorably towards a state of lowest potential energy.

The structure of space, as described by the present model, can be visualized as being analogous to that of soap bubble foam (see Fig. 1). The bubbles in foam correspond to cells in a spatial lattice, and the air molecules within a bubble correspond to photinos within a cell. Whereas its similarities to foam illustrate the architecture of the spatial lattice, the differences between the two give a sense of its behavior. Foam invokes a static view in which bubbles are fixed at their locations relative to their neighbors. They are well insulated from one another and are destroyed when their elastic limits are breached by the pressure exerted upon them. By contrast, a spatial lattice is a dynamic system that evolves with each passing instant in time. Each cell in the lattice is autonomous and active, while the activity of cells entails three essential actions as described below.

First, cells migrate from point to point in the lattice because of the innate compulsion to minimize their potential energy. As the cells within a system approach 


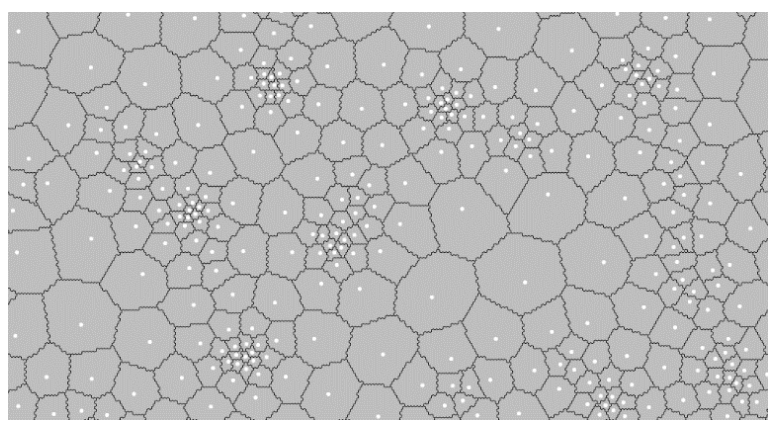

FIG. 1. Cross-sectional view of contiguous cells in a segment of the spatial lattice. The center of mass of each cell is shown as a white dot

equilibrium, a distinct structure emerges in their spatial distribution. The cells cluster strictly in order of nearest density around the highest-density cells in their vicinity, producing a (roughly) concentric symmetry of radially decreasing density around a high-density core in each region within the system (see Fig. 2). A characteristic pattern thus emerges in the density gradient of space at each point in a system, inducing a force to act upon a body when it is in a nonequilibrium state at its location within the system. Compelled to reduce its stored potential energy, the body gains kinetic energy and migrates incrementally towards an equilibrium point in the system where its density conforms to the average density of the ambient space.

Second, a cell is compelled to emit photinos to a neighbor if the total potential energy of the two cells is reduced through this action. This process produces an entropic mechanism such that successive photinos are conducted from cell to neighboring cell within a medium as radiation, when the prevailing conditions permit.

Third, a cell undergoes a phase transition, roughly analogous to plastic deformation, when its elastic limit is breached in conditions of extreme compression or decompression. This action spontaneously minimizes the potential energy of the cell and alters its intrinsic physical character. Phase transition defines the process underlying the formation of ponderable elementary particles, which then cluster in characteristic order to form atomic nuclei and complete atoms of every variety.

The model presented herein demonstrates rigorously that these three cell-level actions are both necessary and sufficient to produce the diverse physical phenomena that are observed at every scale. Together, they endow nature with a teleology that brings about its predictability and permits the generalized laws of conventional theories to be discerned in the behavioral patterns that arise as a consequence.

A guiding principle in the construction of photodynamics is strict adherence to objectivity, which means that any formulation describing an aspect of nature

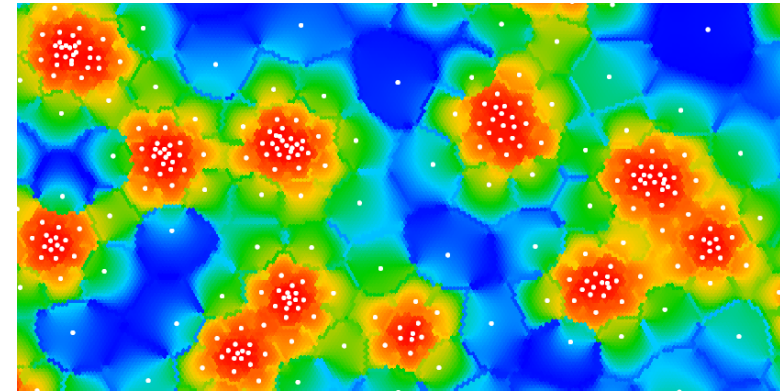

FIG. 2. Two-dimensional schematic of high-density multicell clusters (red) separated by regions of low (blue) and intermediate (yellow and green) density.

is deemed inadmissible if it is inherently abstract or obscure for reasons that cannot, in principle, be known. The description of a phenomenon is further required to satisfy the condition that there exists a well-defined mechanism that enables its physical manifestation.

The inherently abstract character of quantum mechanics renders it incompatible with an objective standard of physical reality [10]. Nevertheless, together with the principles of Heisenberg [11] and Pauli [12], the Schrödinger equation [13-16] undeniably predicts physical effects at the atomic scale with high accuracy, and it is reconstructed herein based explicitly on the commonsense notion that subatomic particles are objectively physical ponderable objects. In addition to the Schrödinger equation, Bohr's formula describing the energy levels of the hydrogen atom [17] is reconstructed within the same framework. Based on these derivations and a new geometric interpretation of the solutions of the Schrödinger equation, the formalism of quantum mechanics is shown to be a mathematically precise allegorical depiction of a much simpler, objective, underlying reality.

Also derived in the framework of the present model are such fundamental relationships as Coulomb's law of electrostatic force, Newton's law of gravity, formulae describing the Compton effect, formulae describing electric and magnetic fields, and other key relationships associated with electromagnetism, atomic theory, optics and thermodynamics. Attractive and repulsive nuclear forces corresponding, respectively, to the strong and weak nuclear forces [18] are shown to arise from a universal electrostatic force that gives rise also to the Coulomb force. A universal function describing the gravitational force between bodies of matter is derived, of which Newton's law is shown to be a limiting case when the medium of space is assumed to be an absolute vacuum.

Dark matter $[19,20]$ is shown to be an ever-present material content that subsists in the very fabric of space everywhere. Contrary to the contemporary hypothesis of dark energy as a source of negative pressure that produces 
the observed expansion of the universe [21], the logic of photodynamics suggests that the current expansion may be one phase among a recurrent cycle of dilation and contraction phases caused by well-defined structural factors. The formation and decay of subatomic particles and the creation of electric charge are each shown to be emergent features that arise from the principles established by the framework of photodynamics. Key physical quantities whose numerical values are derived herein on purely theoretical grounds include the Coulomb constant and hitherto unknown values of the electric dipole moments of electrons and protons.

This paper is divided into ten sections, including the introduction. Sections II, III and IV lay out the theoretical foundations of photodynamics. These sections define, respectively, the fundamental quantities and mathematical relationships of the proposed model, its core principles and cluster dynamics, and the mechanics of the wave function and energy transmission as well as phase transition and other key features. Section V describes the refractive index and its relationship with the wavelength of light in a given medium, and the thermodynamic relationships for temperature and pressure. Section VI describes electromagnetism including the origin of electric charge, the derivation of Coulomb's law as a limiting case of a universal electrostatic force function, and the mechanics of magnetic and electric fields. Section VII describes the origin and dynamics of nuclear forces and nuclear decay, elementary particle formation, and neutron decay. Section VIII reconstructs the Schrödinger equation and Bohr's formula for the hydrogen line spectrum within the altered framework of photodynamics, and shows how the quantum description of the atom allegorically depicts its physical geometry. Section IX describes the derivation of Newton's law of gravity as a limiting case of a more complete universal gravitational force function, the origin of dark matter, and the observed expansion of the universe. Lastly, Section X presents a brief discussion and conclusion.

A computer simulation (as well as a short video) demonstrating the basic logic of photodynamics is available for the reader to download [22]. The simulation permits a range of tests to be conducted under user-defined conditions, and shows graphically the correspondence between some key effects predicted by the proposed model and effects observed in nature.

\section{PRIMARY DEFINITIONS}

\section{A. Physical constants, progression of time, and the unit-action limit}

Five fundamental physical constants of nature are postulated by the theoretical model described in this paper

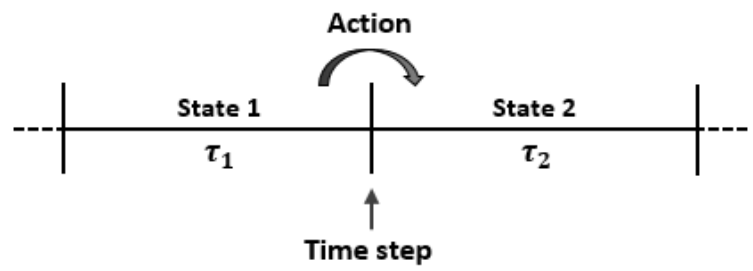

FIG. 3. Change of state in time step separating two consecutive instants $\tau_{1}$ and $\tau_{2}$.

[23], namely Planck's constant $h \approx 6.626 \times 10^{-34} \mathrm{~J} \mathrm{~s}$, the fine-structure constant $\alpha \approx 7.297 \times 10^{-3}$, the elementary charge $e \approx 1.602 \times 10^{-19} \mathrm{C}$, the universal gravitational constant $G \approx 6.672 \times 10^{-11} \mathrm{~m}^{3} \mathrm{~kg}^{-1} \mathrm{~s}^{-2}$, and the instant $\tau \approx 7.372 \times 10^{-51} \mathrm{~s}$. The first four are well-known universal constants of conventional physical theory, while $\tau$ defines the fundamental unit of time, denoting the period separating two consecutive time steps in the temporal evolution of the (recursive) universe. The inverse of $\tau$ is the rate at which the universe evolves, defined herein as the terminal frequency $v_{\tau} \triangleq 1 / \tau \approx$ $1.356 \times 10^{50} \mathrm{~Hz}$.

No other constant that appears in the equations of photodynamics is a fundamental physical constant. Some, such as Boltzmann's constant $k_{B} \approx 1.381 \times 10^{-23} \mathrm{~J} \mathrm{~K}^{-1}$, are proportionality coefficients that harmonize artefactual physical quantities with natural quantities. Others are either system-specific constants (i.e., constants that are not universal, but are applicable to certain types of systems) or they are quantities that are derived from the fundamental constants.

As a natural system of cellular automata, the universe evolves synchronously in discrete, sequential time steps, punctuated by $\tau$. It follows that time is unidirectional, whereby its progression is irreversible. An action is defined herein as any event that transforms the extant state of a cell into a different state, where each action is executed during a time step (see Fig. 3). Consequently, no two consecutive events may occur within a time period shorter than an instant, whereby $\tau$ defines the instantaneous state of the universe.

Moreover, a cell may execute no more than a single action in a single time step. This prohibition against multiple simultaneous actions restricts each cell to a binary choice such that, in any given time step, a cell may execute either a single action or none. This constraint is hereinafter referred to as the unit-action limit.

\section{B. Planck's constant, static energy, and mass-energy equivalence}

Planck's constant is conventionally described as the ratio of the energy of a quantum of electromagnetic radiation to 
its radiation frequency. This implies that radiation energy is deemed a physical reality only when it is actively transmitted from point to point at some frequency. The description is silent regarding the nature of radiation energy in its static state, where it lies latent within a body of matter that has absorbed the received energy.

Departing fundamentally from this conventional description, photodynamics defines Planck's constant as the numerical value of an indivisible quantum of a primordial physical substance that can be best characterized as static energy, and of which every photino is composed. The dimensions of the static energy of photinos at rest thus reflect the dimensions of Planck's constant, namely J s in SI units. It then follows that the active point-to-point transmission of photinos (i.e., of static energy quanta) per unit time connotes the dimensions $(\mathrm{J} \mathrm{s}) / \mathrm{s}=\mathrm{J}$, implying that the transmission of photinos at a given frequency constitutes radiation energy in the conventional sense.

Ontologically, photinos are stipulated to be discrete physical objects and can be described as primordial particles of static energy that morph in shape and size readily to fully occupy the volume of space in which they are confined. Given that a cell represents a unitary photino packet, by definition, cells devoid of photinos cannot exist. Furthermore, because space consists entirely of contiguous cells, a photino can reside only within the body of a host cell. In principle, a cell may contain an unlimited number of photinos. However, in a physical system, the number of photinos resident in any given cell is restricted through their dissipation induced by the entropic process of energy transmission (see Sec. IV C).

According to the logic of the present model, photinos constitute the very source of both inertial mass and radiation energy, as well as the energy that lies latent in space everywhere. While the transmission of photinos from point to point in space constitutes radiation in the conventional sense, the prohibition of light-speed motion by mass-bearing bodies across spatial distances is upheld nevertheless; this is because light propagates not by the physical transport of mass-bearing photinos over distances in a void but rather through the cell-to-cell conduction of a chain of successive photinos. No photino is transported physically any farther than across a single boundary separating two neighboring cells.

Photodynamics describes the energy equivalence of mass as the (potential) dissipation of the total photino content of a body via photino emission at terminal frequency. The cumulative energy $E_{0}$ of a sequence of photinos emitted consecutively at terminal frequency $v_{\tau}$ in unit time $t_{0}$ thus constitutes the energy equivalent of a unit mass $m_{0}$. (Note: $t_{0}=1 \mathrm{~s}$ and $m_{0}=1 \mathrm{~kg}$ in SI units.) Consequently, $E_{0}$ is given by the Planck-Einstein relation [24] for the special case of terminal frequency as

$$
E_{0}=h v_{\tau} \approx 8.988 \times 10^{16} \mathrm{~J} .
$$

Dividing both sides of this equation by $m_{0}$ yields

$$
\frac{E_{0}}{m_{0}}=\frac{h v_{\tau}}{m_{0}}=c^{2}
$$

where $c \approx 2.998 \times 10^{8} \mathrm{~m} \mathrm{~s}^{-1}$ describes the terminal speed of light.

The energy $E_{h}$ transmitted in unit time $t_{0}$ at frequency $v_{0}\left(=1 / t_{0}\right)$ constitutes the energy equivalent of a single photino, which can be stated as

$$
E_{h}=h v_{0} \approx 6.626 \times 10^{-34} \mathrm{~J} .
$$

According to the special theory of relativity $[25,26]$, there exists a universal proportionality between mass and energy, whereby $E_{0} / m_{0}=E_{h} / m_{h}$, where $m_{h}$ is the mass of a photino. Therefore, based on Eq. (2) it can be deduced that $E_{h} / m_{h}=c^{2}$. Replacing $E_{h}$ in this equation with $h v_{0}$ from Eq. (3), the rest mass of a photino can be derived as

$$
m_{h}=\frac{h v_{0}}{c^{2}} \approx 7.372 \times 10^{-51} \mathrm{~kg} .
$$

If a body has mass $m=n m_{h}$, where $n>0$, then its equivalent energy is $E=n E_{h}$. Given that $E_{h} / m_{h}=c^{2}$, it follows that $n E_{h} / n m_{h}=E / m=c^{2}$. Replacing $E_{h}$ in this equation with the right-hand side of Eq. (3) yields the relation $E / m=n h v_{0} / m=c^{2}$, which can be expressed alternatively as

$$
E=n h v_{0}=m c^{2} .
$$

This equation asserts that the equivalence of mass and energy established by Einstein's special theory of relativity extends further to a three-way equivalence between energy $E$, mass $m\left(=E / c^{2}\right)$ and photino content $n h\left(=m c^{2} / v_{0}=E / v_{0}\right)$. In light of the first component of the core hypothesis of the present model, this three-way equivalence further implies that it is the photino content $n h$ that constitutes the source of both mass and energy.

The frequency of a propagating wave can be described as the number of successive wavefronts passing through an imaginary fixed surface per unit time. In photodynamics, the frequency of light $v$ is described as the number $n$ of successive photinos transmitted per unit time across a surface (e.g., the common interface separating two neighboring cells), given as $v \triangleq n / t$, where $t$ is a time interval. The wavelength of light $\lambda$ describes the spatial separation between two successive photinos transmitted to the surface from the same direction at frequency $v$, while $k=2 \pi / \lambda$ represents the associated wave number.

The energy $E$ transported by successive photinos transmitted across a (real or imaginary) surface is thus 
given by the energy equivalent to the total photino content $n h$ transmitted across the surface per unit time, stated as

$$
E=h\left(\frac{n}{t}\right)=h v .
$$

Furthermore, given that $n / t=v$ it follows that the number of photinos transmitted across a surface at frequency $v$ in a unit time interval $t_{0}$ is given as

$$
n=v t_{0}=\frac{v}{v_{0}} .
$$

Based on the classical relation for the speed $u$ of a propagating wave, namely $u=\lambda v$, the limiting wavelength $\lambda_{\tau}$ of a photino conduction wave propagating at terminal frequency can be derived to be

$$
\lambda_{\tau}=\frac{c}{v_{\tau}} \approx 2.211 \times 10^{-42} \mathrm{~m}
$$

It is important to note that the conduction of photinos at $v_{\tau}$ is an imaginary limiting case, where successive photinos are (theoretically) conducted from one cell to the next in each consecutive time step. In a physical system, photino transmission occurs through a conditional entropic mechanism, whereby photino conduction waves propagate at frequencies far lower than $v_{\tau}$ (see Sec. IV C). Consequently, the shortest wavelengths found in nature are some 30 orders of magnitude longer than $\lambda_{\tau}$. As a measure of physical distance, $\lambda_{\tau}$ represents the length of a chord connecting the two points of maximal separation located on opposite sides of a conducting cell. In an idealized case in which cells are approximated as spherical in shape, the maximal chord of a cell is its diameter. (Note that the spatial contiguity of cells dictates that every cell must be roughly polyhedral in shape. However, given that cells may differ greatly in shape from one another, while any given macroscopic region of space typically consists of a very large number of cells, an average cell can be treated logically as approximately spherical.)

Consequently, $\lambda_{\tau}$ represents the maximum average cell diameter that a photino conduction wave would traverse in a single instant if it were to (hypothetically) propagate at terminal frequency $v_{\tau}$. This relationship implies that $\lambda_{\tau}=2 r_{\tau}$, where $r_{\tau} \approx 1.106 \times 10^{-42} \mathrm{~m}$ is the limiting cell radius. In essence, $\lambda_{\tau}$ and $r_{\tau}$ represent respectively the shortest possible wavelength of light and smallest cell radius that nature permits in principle.

The rest mass of an electron is known to be $m_{\mathrm{e}} \approx$ $9.109 \times 10^{-31} \mathrm{~kg}$, equivalent to an energy content of $E_{\mathrm{e}} \approx 8.187 \times 10^{-14} \mathrm{~J}$. The number of photinos $n_{\mathrm{e}}$ contained in an average electron can thus be derived to be

$$
n_{\mathrm{e}}=\frac{E_{\mathrm{e}}}{E_{h}} \approx 1.236 \times 10^{20} .
$$

Similar calculations give estimates of the average numbers of photinos contained in a proton $\left(n_{\mathrm{p}}\right)$ and a neutron $\left(n_{\mathrm{n}}\right)$, namely $n_{\mathrm{p}} \approx 2.269 \times 10^{23}$ and $n_{\mathrm{n}} \approx 2.272 \times 10^{23}$.

\section{Quantitative individuality}

An essential component of the logic of photodynamics is the quantitative individuality of cells within any finite system, which derives from the truism that the physical volume of a body may take any positive numerical value in the real number domain. It follows that the same holds true for the density of a body, which, by definition, is the ratio of the mass (or equivalently the energy or photino content) of a body to its volume.

From the well-known theorem by Cantor on the cardinality of infinite sets [27], which establishes that $|\mathbb{R}|>|\mathbb{N}|$, it can be deduced that $\forall a, b \in \mathbb{R}, a<b$ : $\left|(\mathbb{R} \backslash \mathbb{Q})_{(a, b)}\right| \gg\left|\mathbb{Q}_{(a, b)}\right|$. It therefore follows that $\forall i, j$, $i \neq j, \quad \exists \delta_{i, j} \in \mathbb{R}, \quad \delta_{i, j}>0 \quad: \quad P\left\{\left|\rho_{i}-\rho_{j}\right|=\delta_{i, j}\right\} \gg$ $P\left\{\left|\rho_{i}-\rho_{j}\right|=0\right\}$, where $\rho_{i}$ and $\rho_{j}$ are the densities of any two bodies $i$ and $j$ within a system and $P\{\mathrm{~A}\} \in[0,1]$ is the probability of event A.

In effect, this relationship all but forbids absolute numerical parity between the values of a continuous quantity associated with any two physical bodies in a system, for example, their volumes or densities. Each cell in a finite system is thus rendered distinct, with asymptotic probability one, from every other cell with respect to its density.

Quantitative individuality is vitally important in instilling strict order and structure in the spatial distribution of cells and higher-order bodies of matter in systems at every scale, and it is an essential factor in producing a vast range of physical phenomena such as gravitational and electrostatic forces, atomic structure, electric charge and optical effects.

\section{Density spectrum}

In everyday life, we distinguish objects from one another based on the differences that our senses allow us to discern, such as differences in their shapes, sizes, colors and scents. However, methodical analysis is necessary when the distinction to be made is between two substances irrespective of their forms. One method, known since antiquity, is to compare their densities. In photodynamics, density represents the defining characteristic of material bodies. It is the density of a body that distinguishes it absolutely from all of the other bodies in a system.

Given the hypothesis of the present model that the universe consists entirely of contiguous cells, an absolute 
vacuum in any region of space is precluded by definition. This hypothesis further implies that both matter and space must be composed of cells as their common elemental constituents. The model therefore draws no fundamental distinction between matter and space. Matter is made ponderable and distinct from seemingly empty space solely because the density of its cellular elements is much higher than that of the cells that constitute regions of seemingly empty space. The densities of matter and space thus represent two parts of a continuous spectrum of cell densities.

The density spectrum is closely related to the electromagnetic spectrum of radiation frequencies. Each describes all possible degrees of photino concentration, in a different mode. The density spectrum describes the spatial concentration of photinos resident in a unit volume, while the electromagnetic spectrum describes the temporal concentration of photinos transmitted in unit time. If $V$ is the volume of a cell, then its photino density $\rho$ is defined as

$$
\rho \triangleq \frac{n h}{V}
$$

If $n$ photinos are transmitted to a cell in time $t$, then Eq. (6) gives the radiation energy received by the cell as $E=n h / t$. It follows that the photino density $\rho$ and the radiation energy $E$ describe the total photino content $n h$ concentrated in unit volume and in unit time, respectively.

For practical purposes, the density spectrum can be partitioned into three principal segments - in much the same sense that the electromagnetic spectrum is partitioned, by convention, into several broad segments (e.g., radio waves, visible light, X-rays, etc.).

The uppermost segment of the density spectrum is thus defined as the subspectrum of the highest cell densities. The cells whose densities lie within this subspectrum form the building blocks of multi-cell clusters that constitute high-density atomic-scale particles (e.g., nucleons), where the density of a proton defines the lower end of this subspectrum. These cells are referred to herein as high-density tangible (HDT) cells.

The lowermost segment of the density spectrum is defined as the subspectrum of the lowest cell densities. The cells whose densities lie within the range described by this subspectrum constitute the (single-cell) elements of an intangible medium, namely one that is perceived as empty space. These cells are referred to herein as low-density intangible (LDI) cells.

The intermediate segment of the density spectrum is defined as the subspectrum of cell densities that are lower than those of HDT cells and higher than those of LDI cells. The cells whose densities lie in the range spanned by this subspectrum constitute (relatively) low-density single-cell subatomic particles, namely electrons. These cells are referred to herein as low-density tangible (LDT) cells.

As is the case with the boundaries separating any two neighboring segments in the electromagnetic spectrum (e.g., X-rays and gamma rays), the boundaries separating two neighboring segments in the continuum of the density spectrum cannot be defined precisely. Thus, depending on the context, a cell may be part of either subspectrum if its density lies in a region that broadly defines the boundary between two neighboring subspectra. Moreover, given that the density spectrum spans the full range of all possible densities, the density of every common and exotic species of particles in nature can be classified as part of one of the three subspectra defined above.

Just as our perception of visible light is restricted to a narrow band of frequencies within the electromagnetic spectrum, the present model stipulates that our perception of tangible matter is restricted to the HDT and LDT segments of the density spectrum. An object whose density lies in the LDI segment is thus imperceptible without the aid of scientific instruments; we register it only as the absence of tangible matter. It can be argued that biological evolutionary processes have calibrated our sensory apparatus with these limitations for optimal utility. The survival of our species within its native environment evidently does not require the direct perception of intangible space, much as it does not require the direct visibility of gamma rays or radio waves.

\section{E. Rudimental and ground-state photino content}

The proposed model postulates a rudimental photino content $H_{R}$ for every cell, which is a fundamental quantity defined as the photino content that a cell minimally requires such that $H_{R} \triangleq n_{R} h$, where $n_{R}$ is the rudimental photino count of the cell, while $n_{R} \in \mathbb{R}$ and $n_{R} \geq 1$. The numerical value of $n_{R}$ is set for a cell only during its phase transition under extreme conditions, remaining constant thereafter in all nonextreme conditions. Note that $n_{R}$ (and hence also $H_{R}$ ) lies in the real number domain as a result of the physical mechanism by which its value is set during phase transition (described in Sec. IV B).

Whereas the value of $H_{R}$ is set for a cell through phase transition under extreme conditions, given that photinos come in discrete quanta defined by Planck's constant, in nonextreme conditions the minimum physical photino content of a cell can be equal to $H_{R}$ if and only if $n_{R}$ is an integer. However, because $n_{R} \in \mathbb{R}$, the probability of $n_{R}$ being an integer is vanishingly small. Therefore, the minimum physical photino content of a cell $H_{G}$ is defined as its ground-state photino content, given as $H_{G} \triangleq n_{G} h$, where $n_{G}$ is the ground-state photino count of the cell such that $n_{G} \in \mathbb{N}^{+}$and 


$$
n_{G}=\left\{\begin{array}{ll}
\left\lfloor n_{R}\right\rfloor & \text { if }\left|n_{R}-\left\lfloor n_{R}\right\rfloor\right|<\left|n_{R}-\left\lceil n_{R}\right\rceil\right| \\
\left\lceil n_{R}\right\rceil & \text { if }\left|n_{R}-\left\lfloor n_{R}\right\rfloor\right| \geq\left|n_{R}-\left\lceil n_{R}\right\rceil\right|
\end{array} .\right.
$$

An additional quantity $\eta \triangleq\left(n_{R}-n_{G}\right)$ is defined herein as the difference between the rudimental photino count of a cell and its ground-state photino count, whereby $0 \leq|\eta| \leq 0.5$. A cell can be described as energy neutral if its $n_{R}$ value is such that $\eta=0$. It is energy abundant (denoted as $\eta^{-}$) if $-0.5 \leq \eta<0$ and energy deficient (denoted as $\eta^{+}$) if $0<\eta<0.5$.

As described later, any deviation from energy neutrality induces potential energy in a cell and hence represents a state of energy imbalance. Attaining an ideal state of energy neutrality requires an energy-abundant cell to relinquish a fraction of a photino $\left|\eta^{-}\right| h$ and an energydeficient cell to acquire a fraction of a photino $\left|1-\eta^{+}\right| h$. However, because photinos come only in indivisible units of $h$, fractional photinos do not exist. A state of energy neutrality is thus inherently unattainable for a cell with an energy imbalance. This feature creates a special dynamic at the cellular level that produces the physical effects associated with electric charge (see Sec. VI A).

\section{F. Elastic limits, extreme conditions, and volume expansivity}

The rudimental volume $V_{R}$ of a cell is defined herein as the volume that the cell would occupy if its extant photino content were equal to its rudimental photino content $H_{R}$. As the minimum volume that a cell must occupy, $V_{R}$ defines the lower elastic limit of a cell. A breach of the lower elastic limit of a cell occurs only in conditions of extreme compression, triggering a phase transition and resetting its $V_{R}$ value, which then remains constant in all nonextreme conditions. Furthermore, the rudimental density of a cell is defined as $\rho_{R} \triangleq\left(H_{R} / V_{R}\right)$, while its ground-state density is defined as $\rho_{G} \triangleq\left(H_{G} / V_{R}\right)$.

The par volume $V_{P}$ of a cell is defined herein as the volume that the cell occupies when in a state of elastic equilibrium; $V_{P}$ represents the maximum volume that a cell may occupy, and defines the upper elastic limit of the cell. A breach of the upper elastic limit of a cell occurs only in conditions of extreme decompression, triggering a (reverse) phase transition and resetting its $V_{P}$ value, which then remains constant in all nonextreme conditions.

Extreme conditions are thus defined as conditions in which the compression or decompression of a cell is sufficient to cause its lower or upper elastic limit to be breached. Nonextreme conditions are normal conditions in which neither the compression nor the decompression is sufficient to cause either elastic limit of a cell to be breached.

Let $H_{A}$ be the extant actual photino content of a cell, where $H_{A} \triangleq n_{A} h$, and $n_{A}$ is the actual photino count of the cell such that $n_{A} \in \mathbb{N}^{+}$. Given that $H_{R}$ is defined as the photino content that a cell minimally requires, it follows that $H_{A} \geq H_{R}$. The proposed model postulates that in nonextreme conditions, $V_{P}$ is related to $H_{A}$ such that

$$
\begin{aligned}
V_{P}=V_{R} & +\frac{\alpha}{2}\left(\frac{H_{A}-H_{R}}{\rho_{R}}\right)=V_{R}\left(1+\frac{\alpha}{2}\left(\frac{H_{A}}{H_{R}}-1\right)\right) \\
& =V_{R}\left(1+\frac{\alpha}{2}\left(\frac{H_{A}}{\left(H_{G}+\eta h\right)}-1\right)\right),
\end{aligned}
$$

where $\alpha / 2$ denotes the fundamental coefficient of volume expansivity and $\alpha \approx 1 / 137.036$ is the fine-structure constant. Mechanically, $V_{P}$ is initialized for a cell during a phase transition, when it is set equal to the extant volume of the cell. The quantities $V_{R}, H_{R}$ and $H_{G}$ are then reset for the cell in accordance with Eq. (12), based on the new $V_{P}$ (see Sec. IV B).

\section{G. Elastic potential energy}

If $V_{A}$ is the extant actual volume of a cell, then its divergence from the equilibrium volume is $\left(V_{P}-V_{A}\right)$. In accordance with the law of elasticity, any divergence from its equilibrium volume induces elastic potential energy $U_{V}$ in a cell such that $U_{V} \propto\left(V_{P}-V_{A}\right)^{2} / 2$, which can be stated as $U_{V}=\left(k_{V} / 2\right)\left(V_{P}-V_{A}\right)^{2}$, where $k_{V} \triangleq v_{0}\left(\rho_{A} / V_{A}\right)$ is posited to be a case-specific proportionality coefficient and $\rho_{A} \triangleq H_{A} / V_{A}$ is the extant actual photino density of the cell. The elastic potential energy of a cell is thus given as

$$
U_{V}=\frac{v_{0}}{2}\left(\frac{\rho_{A}}{V_{A}}\right)\left(V_{P}-V_{A}\right)^{2}=\frac{H_{A} v_{0}}{2}\left(\frac{V_{P}}{V_{A}}-1\right)^{2},
$$

where the ratio $\kappa \triangleq\left(V_{P} / V_{A}\right)$ describes the compression of the cell.

\section{FRAMEWORK}

\section{A. Autonomic principle}

The autonomic principle can be described as the universal propensity of bodies to minimize their stored potential energy. In its broadest sense, the potential energy of a body may appear in any of its various forms, such as the stored potential energy of a stretched spring, the gravitational potential energy of a pendulum at full swing or the elastic potential energy of a compressed tennis ball or cell.

The present model describes the potential energy of a body in each of its various forms as an extension of the potential energy generated according to the laws of elasticity, and it is defined herein as the mechanical energy induced in a body by the displacement of an associated 
physical quantity $\mathfrak{R}$ from its equilibrium value $\Re_{\text {eq }}$, if there exists a value $\mathfrak{R}_{\text {eq }}$ that the body seeks to attain.

Consequently, irrespective of the scale and nature of the body and the nature of $\mathfrak{R}$, the stored potential energy $U_{\mathfrak{R}}$ of the body is defined by the universal function

$$
U_{\Re} \triangleq \frac{1}{2} k_{\Re}\left(\Re-\Re_{e q}\right)^{2},
$$

where $k_{\Re}$ is a case-specific coefficient of proportionality that describes the degree of resistance to the displacement of $\mathfrak{R}$ from its equilibrium value $\mathfrak{R}_{e q}$. The autonomic principle can then be stated symbolically as

$$
U_{\Re} \rightrightarrows 0
$$

where the notation $x \rightrightarrows \mathfrak{X}$ connotes maximal change per time step in the numerical value of a quantity $x$ towards a terminal value $\mathfrak{X}$. This propensity further implies that the autonomic principle induces resistance to an increase in the value of $U_{\Re}$.

In accordance with classical laws governing the behavior of elastic bodies and systems, for which the fundamental principle is defined by Hooke's law of elasticity, under nonextreme conditions $U_{\mathfrak{R}}$ produces a restoring force $\overrightarrow{\mathcal{F}}$ that acts on a body such that

$$
\overrightarrow{\mathcal{F}}=-k_{\mathfrak{R}} \overrightarrow{\mathfrak{D}}
$$

where $\overrightarrow{\mathfrak{D}}$ is a measure of the displacement of the body from equilibrium.

Three distinct forms of potential energy may exist simultaneously at the cellular level, namely (i) $U_{V}$ due to the volumetric nonequilibrium of a cell, (ii) $U_{\eta}$ due to the energy imbalance of a cell (see Sec. VI A), and (iii) $U_{g}$ due to the displacement of a cell from its equilibrium location within the ambient system (see Sec. IX B). The total potential energy $U$ of a cell is therefore given as

$$
U \triangleq U_{V}+U_{\eta}+U_{g}
$$

where the autonomic principle dictates that $U \rightrightarrows 0$.

A cell is defined to be in a state of absolute equilibrium when $U=0$. While the autonomic principle dictates that every cell must seek a state of absolute equilibrium, in nonextreme conditions such a state is all but precluded by quantitative individuality and other constraints that are ever-present in a physical system. Therefore, in nonextreme conditions, a cell is defined to be in its (optimal) equilibrium state when $U$ is minimal, expressed symbolically as

$$
U \simeq 0
$$

where the notation $x \simeq \mathfrak{X}$ describes hereinafter an optimal state such that the extant numerical value of a quantity $x$ is as close as possible to its terminal value $\mathfrak{X}$.

\section{B. Elasticity of cells and nonequilibrium dynamics}

By virtue of its innate elasticity, a cell is compelled to seek elastic equilibrium when in a nonequilibrium state, given as $V_{A} \neq V_{P}$. From Eq. (13), a nonequilibrium state implies that $U_{V}>0$. Therefore, every cell seeks to reduce its elastic potential energy maximally in each time step when its extant volume differs from its par volume. The autonomic principle with respect to the elastic potential energy of a cell can therefore be stated as

$$
U_{V}=\frac{H_{A} v_{0}}{2}\left(\frac{V_{P}}{V_{A}}-1\right)^{2} \rightrightarrows 0
$$

Furthermore, if the constituent cells of a system are in a nonequilibrium state, then the system as a whole is in a nonequilibrium state. Thus, every system is at all times compelled to reduce its total elastic potential energy maximally in each time step, given as $\sum_{i=1}^{N} U_{V, i} \rightrightarrows 0$, where $U_{V, i}$ is the elastic potential energy of cell $i$ within a system composed of $N$ cells.

As described below, reduction in its elastic potential energy requires a cell to execute one of three possible actions in any given time step, namely physical motion, photino emission or a phase transition. Multiple actions by a cell in a single time step are prohibited by the unit-action limit. In principle, the volumetric state of a cell satisfies $V_{R} \leq V_{A} \leq V_{P}$ in nonextreme conditions. Under extreme compression, the lower elastic limit of a cell is breached when its volumetric state satisfies $V_{A}<V_{R}$; while under extreme decompression, the upper elastic limit of a cell is breached when its volumetric state satisfies $V_{A}>V_{P}$.

No action ensues if a cell is in a state of elastic equilibrium, for then $V_{A} \simeq V_{P}$ and $U_{V} \simeq 0$. It follows that a cell can execute an action if and only if it is in a nonequilibrium state. From Eq. (19), any change in $U_{V}$ requires a change in one or more of its arguments $H_{A}, V_{P}$ and $V_{A}$. In addition, from Eq. (12), any change in $V_{P}$ must be accompanied by offsetting changes in one or more of its arguments $V_{R}, H_{A}$ and $H_{R}$. However, because $V_{R}$ and $H_{R}$ are reset only during the phase transition of a cell in extreme conditions, in nonextreme conditions a change in $V_{P}$ can occur if and only if there is a change in $H_{A}$.

Consequently, in nonextreme conditions, any change in $U_{V}$ requires a change in either $V_{A}$ or $H_{A}$, whereby a cell whose elastic potential energy exceeds the minimal value is compelled by the autonomic principle to execute one of the following two possible actions in any given time step. 
$V_{A} \rightrightarrows V_{P}$. Through this action, a cell seeks to maximize its physical volume by dilating into the surrounding space. However, the ability of a cell to dilate is constrained by the spatial limitations imposed by the neighboring cells, this being because its neighbors are also subject to the autonomic principle and seek to maximize their own volumes while being constrained by the overall spatial limitations of the local system. These competing propensities produce a mechanism whereby cells are compelled to relocate in collaborative action to reduce their own elastic potential energies maximally, thereby reducing that of the system as well. It is this mechanism that governs the point-to-point migration of cells in a system (see Sec. III C).

$H_{A} \rightrightarrows H_{G}$. Through this action, a cell seeks to minimize its photino content by emitting photinos to a neighbor. However, the ability of a cell to emit photinos requires a neighbor to be capable of absorbing the emitted photinos, this being because each neighboring cell is itself subject to the autonomic principle and is therefore compelled to reduce its own photino content maximally. These competing impulses produce an entropic mechanism whereby photinos are transmitted from cell to cell when certain well-defined conditions are satisfied. This process is responsible for the transmission of energy within a medium (see Sec. IV C).

The autonomic principle further dictates that, of these two possible actions, the one executed by a cell in any given time step under nonextreme conditions must be the action that produces the greatest reduction in its elastic potential energy.

In extreme conditions, the autonomic principle enforces changes in the values of key physical quantities associated with a cell, thereby spontaneously altering its state such that $U_{V}=0$. These changes are irreversible in nonextreme conditions. The mechanism of this process is as follows.

$V_{P}=V_{A}$ and $\rho_{R}=\rho_{A}$. The par volume of a cell is set equal to its extant volume and its rudimental density is set equal to its extant density. In accordance with Eq. (12), this action recalibrates $V_{R}, H_{R}$ and $H_{G}$. This cascade of changes in the intrinsic values of the key physical quantities associated with the cell constitutes the essential mechanism of phase transition (see Sec. IV B). It alters fundamentally the physical character of the cell and gives rise to the creation of electric charge, as well as the process of subatomic particle formation, among other atomic-scale physical effects.

\section{Cell motion}

According to the autonomic principle, if $U_{V}>0$ then it follows that $U_{V} \rightrightarrows 0$. In nonextreme conditions, the only volumetric state that can cause $U_{V}>0$ is when a cell is compressed such that $V_{A}<V_{P}$. As implied by Eq. (19), if $H_{A}$ is held constant then any reduction in the $U_{V}$ of a cell can occur only if $V_{A} \rightrightarrows V_{P}$, which requires the cell to dilate maximally in each time step. Because the cells within a system are spatially contiguous, the dilation of a cell requires its neighboring cells to surrender a portion of the space that they occupy by contracting in volume. However, such forced volume contraction increases the compression of the neighboring cells, thus increasing their $U_{V}$, which they are in turn compelled to resist in accordance with the autonomic principle. Therefore, the neighboring cells impose spatial constraints that inhibit the ability of a cell to dilate in volume in pursuit of elastic equilibrium and thus towards its equilibrium state $U_{V} \simeq 0$. However, the total elastic potential energy of a system can nevertheless be reduced in each time step if groups of neighboring cells within the system rotate in concert about a common axis such that the volume of each cell within each group is maximized. Such group-wise in-concert rotations define the mechanism of cell motion within a system. This process reduces the total potential energy of the system incrementally, and it terminates when all of the constituent cells in the system are maximal in volume, namely when $V_{A} \simeq V_{P}$, whereby $U_{V} \simeq 0$ for every cell in the system.

Given the spatial contiguity of cells, when cell A shifts partly from its extant location to a neighboring location, it must partly displace cell B occupying that location. In addition, the space partly vacated by cell $\mathrm{A}$ must be filled by cell $\mathrm{C}$ from another neighboring location. Cell motion thus entails the simultaneous rotation of a group of neighboring cells, such as a triplet, about a common axis, while the sole condition for such motion is maximal reduction in $U_{V}$ as described by Eq. (19).

A two-dimensional plane permits two degrees of freedom, whereby the rotation of a triplet may be either clockwise or counterclockwise. Three-dimensional space permits multiple degrees of freedom, whereby a group of conjoined neighbors, which might be a pair, triplet, quadruplet or higher-order group, may rotate in any number of ways. However, the logic of triple-cell rotation in a two-dimensional plane applies equally to the rotation of higher-order cell groups in three-dimensional space, notwithstanding the greater complexity of the latter. Described next is the mechanism of a triple-cell rotation.

Figure 4 shows a triplet $\{\mathrm{A}, \mathrm{B}, \mathrm{C}\}$ rotating counterclockwise in a two-dimensional plane. Given its two degrees of freedom, the rotation may also be clockwise. Let $S_{\mathrm{ABC}}\left(U_{V}\right)=U_{V}(\mathrm{~A})+U_{V}(\mathrm{~B})+U_{V}(\mathrm{C})$, $S_{\mathrm{ABC}}^{\prime}\left(U_{V}\right)=U_{V}^{\prime}(\mathrm{A})+U_{V}^{\prime}(\mathrm{B})+U_{V}^{\prime}(\mathrm{C})$ and $S_{\mathrm{ABC}}^{\prime \prime}\left(U_{V}\right)=$ 
$U_{V}^{\prime \prime}(\mathrm{A})+U_{V}^{\prime \prime}(\mathrm{B})+U_{V}^{\prime \prime}(\mathrm{C})$, where $S_{\mathrm{ABC}}\left(U_{V}\right)$ is the total potential energy of $\{\mathrm{A}, \mathrm{B}, \mathrm{C}\}$ prior to rotation, $S_{\mathrm{ABC}}^{\prime}\left(U_{V}\right)$ is its total potential energy after one counterclockwise turn and $S_{\mathrm{ABC}}^{\prime \prime}\left(U_{V}\right)$ is its total potential energy after one clockwise turn (which, in the illustration, is equivalent to two counterclockwise turns). Note that a single turn in the rotation of a triplet does not imply the movement of an entire cell into space previously occupied by a neighbor. The triplet rotates incrementally, in small step-wise movements. Each turn occurs in a single time step and entails a fractional part of each cell moving into a fractional space vacated by a neighbor. The center of mass of each cell in the triplet thus shifts incrementally. Figure 4 shows two turns, where the centers of mass of cells in the triplet, $c_{\mathrm{A}}, c_{\mathrm{B}}$ and $c_{\mathrm{C}}$ shift incrementally over two time steps, namely $(t+1)$ and $(t+2)$.

The autonomic principle dictates that the rotation of triplet $\{\mathrm{A}, \mathrm{B}, \mathrm{C}\}$ is possible if and only if $S_{\mathrm{ABC}}\left(U_{V}\right)>$ $S_{\mathrm{ABC}}^{\prime}\left(U_{V}\right)$ for a counterclockwise turn or $S_{\mathrm{ABC}}\left(U_{V}\right)>$ $S_{\mathrm{ABC}}^{\prime \prime}\left(U_{V}\right)$ for a clockwise turn. Let $\mathcal{A}$ be a set whose elements are cell $i$ and all of its neighbors. In each turn, $i$ must form an optimal triplet $\mathcal{O}_{i j k}$ with two neighbors $j$ and $k$ selected from all of the candidate cells in $\mathcal{A}$ such that a single (clockwise or counterclockwise) turn maximally reduces the total potential energy of the triplet. This action can be described as

$$
\mathcal{O}_{i j k}=\underset{i, j, k \in \mathcal{A}}{\arg \max }\left(\begin{array}{c}
S_{i j k}\left(U_{V}\right)-S^{\prime}{ }_{i j k}\left(U_{V}\right), \\
S_{i j k}\left(U_{V}\right)-S^{\prime \prime}{ }^{\prime}{ }_{j k k}\left(U_{V}\right)
\end{array}\right) .
$$

If $\mathcal{O}_{\mathrm{ABC}}$ is an optimal triplet of cells $\{\mathrm{A}, \mathrm{B}, \mathrm{C}\}$, then by the unit-action limit $\mathcal{O}_{\mathrm{ABC}}$ is an isolated system for the duration of each turn. By the law of conservation of energy, and as an isolated system during each turn, any reduction in the total potential energy of $\mathcal{O}_{\mathrm{ABC}}$ during a turn of the triplet must be converted into kinetic energy.

Let $t_{\mathrm{ABC}}$ be the time required for one full rotation of $\mathcal{O}_{\mathrm{ABC}}$, given as $t_{\mathrm{ABC}} \triangleq n_{\mathrm{ABC}} \tau$, where $n_{\mathrm{ABC}}$ is the number of elapsed instants. It follows that $t_{\mathrm{ABC}}$ is a function of the kinetic energy of $\mathcal{O}_{\mathrm{ABC}}$, which equals the reduction in its total potential energy. The following formula describes the general case in which $S_{\mathrm{ABC}}\left(U_{V}\right)>S^{\prime}{ }_{\mathrm{ABC}}\left(U_{V}\right)$ :

$$
\begin{aligned}
& S_{\mathrm{ABC}}\left(U_{V}\right)-S^{\prime}{ }_{\mathrm{ABC}}\left(U_{V}\right) \\
& =\left(\frac{m(\mathrm{~A})\left|\vec{v}_{\mathrm{A}}\right|^{2}}{2}+\frac{m(\mathrm{~B})\left|\vec{v}_{\mathrm{B}}\right|^{2}}{2}+\frac{m(\mathrm{C})\left|\vec{v}_{\mathrm{C}}\right|^{2}}{2}\right),
\end{aligned}
$$

where $m(\mathrm{~A}), m(\mathrm{~B})$ and $m(\mathrm{C})$ denote the respective masses of cells A, B and C, while $\vec{v}_{\mathrm{A}}, \vec{v}_{\mathrm{B}}$ and $\vec{v}_{\mathrm{C}}$ are their respective velocities during the rotation of $\mathcal{O}_{\mathrm{ABC}}$. The velocities of the cells are given by $\vec{v}_{\mathrm{A}}=\left(\vec{c}_{\mathrm{C}}-\vec{c}_{\mathrm{A}}\right) / t_{\mathrm{ABC}}$, $\vec{v}_{\mathrm{B}}=\left(\vec{c}_{\mathrm{A}}-\vec{c}_{\mathrm{B}}\right) / t_{\mathrm{ABC}}$ and $\vec{v}_{\mathrm{C}}=\left(\vec{c}_{\mathrm{B}}-\vec{c}_{\mathrm{C}}\right) / t_{\mathrm{ABC}}$, where $\vec{c}_{\mathrm{A}}, \vec{c}_{\mathrm{B}}$ and $\vec{c}_{\mathrm{C}}$ are radius vectors of the centers of mass of

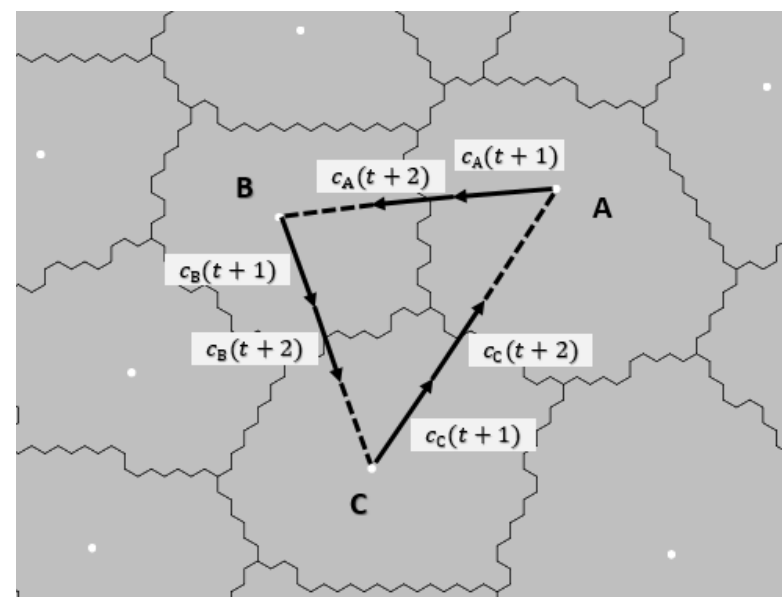

FIG. 4. Step-wise triple cell rotation.

cells A, B and C, respectively. Equation (21) can therefore be written as

$$
\begin{aligned}
& S_{\mathrm{ABC}}\left(U_{V}\right)-S^{\prime}{ }_{\mathrm{ABC}}\left(U_{V}\right) \\
& =\left(\frac{m(\mathrm{~A})\left|\left(\vec{c}_{\mathrm{C}}-\vec{c}_{\mathrm{A}}\right)\right|^{2}}{2 t_{\mathrm{ABC}}^{2}}+\frac{m(\mathrm{~B})\left|\left(\vec{c}_{\mathrm{A}}-\vec{c}_{\mathrm{B}}\right)\right|^{2}}{2 t_{\mathrm{ABC}}^{2}}\right. \\
& \left.\quad+\frac{m(\mathrm{C})\left|\left(\vec{c}_{\mathrm{B}}-\vec{c}_{\mathrm{C}}\right)\right|^{2}}{2 t_{\mathrm{ABC}}^{2}}\right)
\end{aligned}
$$

whereby

$$
\begin{aligned}
t_{\mathrm{ABC}}=\left[\left(m(\mathrm{~A})\left|\left(\vec{c}_{\mathrm{C}}-\vec{c}_{\mathrm{A}}\right)\right|^{2}+m(\mathrm{~B})\left|\left(\vec{c}_{\mathrm{A}}-\vec{c}_{\mathrm{B}}\right)\right|^{2}\right.\right. \\
\left.+m(\mathrm{C})\left|\left(\vec{c}_{\mathrm{B}}-\vec{c}_{\mathrm{C}}\right)\right|^{2}\right) \\
\\
\left./ 2\left(S_{\mathrm{ABC}}\left(U_{V}\right)-S^{\prime}{ }_{\mathrm{ABC}}\left(U_{V}\right)\right)\right]^{1 / 2}
\end{aligned}
$$

If $\varsigma_{\mathrm{ABC}}$ is the rotational speed of $\mathcal{O}_{\mathrm{ABC}}$, where $\varsigma_{\mathrm{ABC}} \triangleq$ $1 / t_{\mathrm{ABC}}$, then

$$
\begin{aligned}
S_{\mathrm{ABC}}= & {\left[2\left(S_{\mathrm{ABC}}\left(U_{V}\right)-S^{\prime}{ }_{\mathrm{ABC}}\left(U_{V}\right)\right)\right.} \\
& /\left(m(\mathrm{~A})\left|\left(\vec{c}_{\mathrm{C}}-\vec{c}_{\mathrm{A}}\right)\right|^{2}\right. \\
& +m(\mathrm{~B})\left|\left(\vec{c}_{\mathrm{A}}-\vec{c}_{\mathrm{B}}\right)\right|^{2} \\
& \left.\left.+m(\mathrm{C})\left|\left(\vec{c}_{\mathrm{B}}-\vec{c}_{\mathrm{C}}\right)\right|^{2}\right)\right]^{1 / 2} .
\end{aligned}
$$

Because the lifetime of an optimal triplet $\mathcal{O}_{\mathrm{ABC}}$ is a single turn, each cell must form a new optimal triplet following the completion of each turn. Consequently, the point-to-point migration of a cell terminates when it can no longer form a new triplet whose rotation would further reduce the elastic potential energy of the triplet. The overall migration speed of a cell is then a function of the average rotational speed of the triplets that the cell forms over the course of its migration. 


\section{Nearest-density order}

Suppose that a system $\mathcal{B}$ composed of $N$ cells has a fixed volume $V(\mathcal{B})$ such that $V(\mathcal{B})=(1 / 2) \sum_{i=1}^{N} V_{P, i}$, where $i \in \mathcal{B}$. The cells in $\mathcal{B}$ are then subject to compression whereby, on average, $V_{A, i}=(1 / 2) V_{P, i}$. If $V_{R, i}, H_{A, i}$ and $H_{R, i}$ of cell $i$ are known, then $V_{P, i}$ can be determined precisely from Eq. (12). Furthermore, because $V_{A, i} \rightrightarrows V_{P, i}$, if the constituent cells of the system are in equilibrium relative to one another, then $V_{A, i}$ must be maximal for each cell in $\mathcal{B}$. However, despite having precise knowledge about the quantities associated with $i$ and the conditions prevailing in its environment, the proportion of $V(\mathcal{B})$ that must actually be allocated to the cell as its physical volume $V_{A, i}$ is unknown. If, for instance, $V_{P, i}$ differs greatly from the corresponding values for the other cells in $\mathcal{B}$, then by what rule or criterion is $V_{A, i}$ determined such that $V_{A}$ is maximized for every cell in $\mathcal{B}$ ?

The answer to this essential question lies in the aphorism that nature admits no inequities. No cell has entitlement to spatial resources that every other cell in a system does not have. Based on the fundamental hypothesis that photinos alone constitute the ultimate elements of space, the proposed model stipulates that each cell within a system is allocated its share of the total available volume of space in proportion to its extant photino content. Thus, cells of high density, which require proportionately small volumes while possessing high photino contents, are allocated space in excess of the volumes they maximally require, and cells of low density may be allocated less space than they require. Somewhat counterintuitively, it is from this very imbalance between spatial allocation and spatial requirement that an optimal order of cell arrangement emerges and gives rise to a distinct structure in the distribution of cells within a system.

Let $\mathcal{B}$ now represent the general case of a system whose $N$ cells are distributed randomly within an imaginary closed surface of arbitrary extent. Then $\sum_{i=1}^{N} H_{A, i}$ is the total photino content of the system and $\sum_{i=1}^{N} V_{A, i}$ is its total volume. The extant photino content of a given cell $c$ is $H_{A, c}$, while its volume yet to be determined is $V_{A, c}$. The pro rata allocation to cell $c$ of its due share of the total volume of the system is therefore given by

$$
\frac{V_{A, c}}{\sum_{i=1}^{N} V_{A, i}}=\frac{H_{A, c}}{\sum_{i=1}^{N} H_{A, i}} \Rightarrow \rho_{c}=\rho_{\mathcal{B}} .
$$

Thus, a (finite) system is in its ideal state when the density distribution within it is isotropic. If $\rho_{\mathcal{B}^{\prime}}$ is the photino density of a two-cell system $\mathcal{B}^{\prime}$ composed of neighboring cells $i$ and $j$ such that $\mathcal{B}^{\prime} \in \mathcal{B}$, then from Eq. (25) it follows that $\rho_{i}=\rho_{\mathcal{B}^{\prime}}$, which can be stated in expanded form as

$$
\frac{H_{A, i}}{V_{A, i}}=\frac{\left(H_{A, i}+H_{A, j}\right)}{\left(V_{A, i}+V_{A, j}\right)} \Rightarrow \rho_{i}-\rho_{j}=0 .
$$

Hence, if a system is in its ideal state, then every cell in the system must have the same density. However, such a state cannot exist in a physical system because numerical parity between the densities of any two cells in a system is prohibited, with asymptotic probability one, by quantitative individuality. Consequently, there exists a potential energy $U_{\mathcal{B}^{\prime}}$ in each $\mathcal{B}^{\prime}$ such that $U_{\mathcal{B}^{\prime}}=$ $\left(k_{\mathcal{B}^{\prime}} / 2\right)\left(\rho_{i}-\rho_{j}\right)^{2}$, where $k_{\mathcal{B}^{\prime}}$ is a system-specific coefficient. Given that $U_{\mathcal{B}^{\prime}} \rightrightarrows 0$, a universal propensity is induced such that any given two-cell system must tend maximally towards its ideal state, which can be expressed as $\left|\rho_{i}-\rho_{j}\right| \rightrightarrows 0$. It follows that $\mathcal{B}$ is as close as possible to its ideal state if and only if each cell $i$ forms an optimal pair $\mathcal{P}_{i j}$ with a neighbor $j$, where

$$
\mathcal{P}_{i j}=\underset{i, j \in \mathcal{B}}{\arg \min }\left|\rho_{i}-\rho_{j}\right|
$$

When the elements of a system are arranged such that there exists an optimal set $\mathcal{B}^{\prime \prime}=\left\{\mathcal{P}_{i j}\right\}$ for all $i, j \in \mathcal{B}$, then $\mathcal{B}^{\prime \prime}$ represents a system in an equilibrium state. This is because it is only when all of the cells in $\mathcal{B}$ are thus arranged that the system is as close as possible to its ideal state and its total elastic potential energy $U_{V}(\mathcal{B}) \simeq 0$.

A system thus attains an equilibrium state when all of the cells within it are arranged in nearest-density order such that $\forall i, j \in \mathcal{B}, \forall k \neq i, j \in \mathcal{B}:\left|\rho_{i}-\rho_{j}\right|<\left|\rho_{i}-\rho_{k}\right|$. Thus, the state of equilibrium in any given system $\mathcal{B}$ is defined by the condition

$$
\left(\rho_{i}-\rho_{j}\right) \simeq 0
$$

for all $i$ and $j$.

The autonomic principle thus creates a compulsion in the constituent cells of a system to cluster together such that they are arranged precisely in order of nearest density relative to one another.

\section{E. Spatial density distribution and gradient}

The order according to nearest density instills a strict sequence in the arrangement of cells relative to one another as a system reaches equilibrium. A onedimensional case of this arrangement can be described by a string of cells in which the highest-density cell lies at one end and the lowest-density cell lies at the other end, while the density of the intervening cells decreases sequentially from one end of the string to the other. In a string where the highest-density cell is located at the center, nearestdensity order requires that cell densities must decrease sequentially in both directions away from the central cell. 
This arrangement describes a one-dimensional Gaussian (i.e., normal) distribution of cell densities.

In three-dimensional physical space, the compulsion of cells to self-organize in nearest-density order dictates that the distribution of cell densities in any given region must follow a corresponding structure, namely a threedimensional Gaussian density distribution. The highestdensity cells thus collectively form the core of a roughly spherically symmetric cell cluster in each region of a system. Cell densities within each cluster then decrease sequentially away from the high-density core radially in each direction (see Fig. 2).

This (roughly) concentric symmetry in the spatial density distribution around a high-density core transcends scale and extends to any physical system whose elements are, at least largely, in an equilibrium state. Regardless of the scale of a system, an approximate three-dimensional Gaussian symmetry thus emerges in the density distribution of the elements of the medium surrounding a high-density primary body (denoted hereinafter by $\oplus$ ) that forms the core of the system. (Note: This emergent spatial structure is demonstrated graphically in the computer simulation provided herewith [22]. It is, however, important to be cognizant of the limitations of the simulation arising above all from the fact that, even in principle, a computer cannot reproduce the infinite precision of nature in the measurement of density differences between cells, nor the speeds of up to $v_{\tau} \sim 10^{50} \mathrm{~Hz}$ at which processes in nature can occur.)

A point on an imaginary spherical surface $\mathcal{S}$ in such a system can be described by its spherical coordinates $(r, \theta, \phi)$, where the radial coordinate $r$ is measured relative to the center of mass of $\oplus$, while $\theta$ and $\phi$ are measured relative to the polar and azimuthal axes, respectively, of the system enclosed in $\mathcal{S}$ (see Fig. 5). Note: The mechanism that produces polar and azimuthal axes is described in Sec. IV A.

If $\hat{\mathbf{r}}$ is a radial unit vector outwardly normal to $\mathcal{S}$, then the density gradient at a point $(r, \theta, \phi)$ on $\mathcal{S}$ is given by

$$
\vec{\nabla} \rho=\hat{\mathbf{r}} \frac{\partial \rho}{\partial r}+\widehat{\boldsymbol{\theta}} \frac{1}{r} \frac{\partial \rho}{\partial \theta}+\widehat{\boldsymbol{\phi}} \frac{1}{r \sin \theta} \frac{\partial \rho}{\partial \phi},
$$

where $\widehat{\boldsymbol{\theta}}$ and $\widehat{\boldsymbol{\phi}}$ are the polar and azimuthal unit vectors, respectively. Given the approximate Gaussian symmetry in the density distribution of the elements of a system, in macroscopic systems the variation in the density of the elements located at points on the surface $\mathcal{S}$ tends to be minor in the directions of $\widehat{\boldsymbol{\theta}}$ and $\widehat{\boldsymbol{\phi}}$ as compared to the density variation in the direction of $\hat{\mathbf{r}}$. Consequently, $\partial \rho / \partial \theta \approx \partial \rho / \partial \phi \approx 0$, whereby the medium surrounding a macroscopic primary body tends to have an appreciable density gradient only in the radial direction, and Eq. (29) reduces to

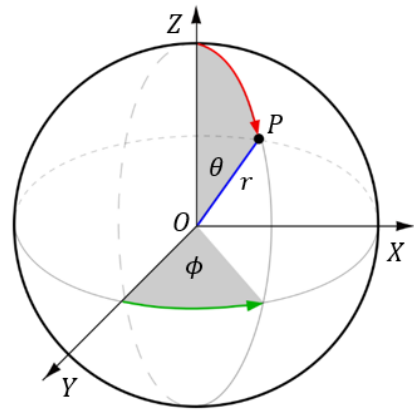

FIG. 5. Spherical coordinates $(r, \theta, \phi)$ of a point $P$ relative to origin $O$, where $r$ is the radial coordinate, $\theta$ is the polar coordinate and $\phi$ is the azimuthal coordinate.

$$
\vec{\nabla} \rho \approx \hat{\mathbf{r}} \frac{\partial \rho}{\partial r}
$$

Note that a sinusoidal order in the sequence of cell densities is consistent with the nearest-density order of cell arrangement. This is because a sinusoidal pattern is simply a sequence in which cells are arranged in nearest density order such that their densities alternate cyclically about a mean value. Substructures within two-dimensional surfaces and three-dimensional spatial regions may therefore contain undulating patterns of cell density. They may form filaments, streamlines, intersecting wavelike structures, or other complex wave patterns. There may exist fractal substructures, where self-similar spatial density patterns repeat at multiple levels of diminishing scale [28].

Any number of wavelike spatial density patterns that satisfy the requirement of nearest-density order may therefore coexist as substructures within a system. However, the two fundamental patterns that predominate as overall density distribution structures in natural systems are (a) three-dimensional Gaussian, where the elements of the system are arranged in roughly concentric radially decreasing density order around a high-density core, and (b) one-dimensional sinusoidal, where the system is a string composed of a chain of segments whose elements (which may individually be cells, cell clusters, macroscopic bodies or large-scale systems) are arranged roughly in order of sequentially reducing density around the highest-density elements in each segment.

A key consequence of the characteristic Gaussian density distribution inside a system enclosed by $\mathcal{S}$ is that the distribution of spatial density surrounding any given element within $\mathcal{S}$ (e.g., a cell) must be anisotropic if the system is in equilibrium. This is because the density of the medium inside $\mathcal{S}$ increases progressively in the direction of the primary body. Consequently, for any given element within $\mathcal{S}$, the density of its immediate neighbor on the side facing the primary body must be greater than that of its neighbor on the diametrically opposite side facing 
antiparallel to the direction of the primary body. Meanwhile, the densities of all other immediate neighbors of the element must increase sequentially from the lower density side (facing antiparallel to the direction of the primary body) toward the diametrically opposite higher density side (facing the primary body).

In the framework of photodynamics, this ever-present anisotropy in the density distribution surrounding each cell in microscopic systems underpins wave function mechanics (see Sec. IV A), which, in turn, is central to the theoretical foundations of atomic physics. Furthermore, the anisotropic density distribution of the cells that make up the intangible medium surrounding a celestial body is a key factor in determining the gravitational behavior of bodies at the cosmic scale (see Sec. IX D).

\section{F. Compactness}

If a system enclosed by an imaginary spherical surface $\mathcal{S}$ of radius $r$ is centered on a primary body $\oplus$, then the radially decreasing density distribution of the elements surrounding $\oplus$ implies that the overall density of the system inside $\mathcal{S}$ also decreases somewhat with each increment in $r$. Given further that the surface area of $\mathcal{S}$ increases as a function of its radius $r$, it follows that the density of the system enclosed in $\mathcal{S}$ decreases with each increment in the surface area of $\mathcal{S}$ as well. The density $\rho_{\mathcal{S}}$ of a system whose elements are in equilibrium is thus stipulated to vary as the inverse of its surface area $A_{\mathcal{S}}$, expressed as $\rho_{\mathcal{S}} \propto 1 / A_{\mathcal{S}}$. If $p_{\mathcal{S}}^{\prime}$ is a system-specific proportionality coefficient associated with this relation, then $\rho_{\mathcal{S}}=p_{\mathcal{S}}^{\prime} / A_{\mathcal{S}}$, which can be restated as $p_{\mathcal{S}}^{\prime}=\rho_{\mathcal{S}} A_{\mathcal{S}}$.

If $\grave{\rho}_{\mathcal{S}} \triangleq \rho_{\mathcal{S}}\left(v_{0} / c^{2}\right)$, then based on Eq. (5) this relationship can be stated as $\grave{\rho}_{\mathcal{S}}=m_{\mathcal{S}} / V_{\mathcal{S}}$, which describes the mass density of the system inside $\delta$. Likewise, if $p_{\mathcal{S}} \triangleq$ $p_{\mathcal{S}}^{\prime}\left(v_{0} / c^{2}\right)$, then $p_{\mathcal{S}}$ describes the compactness of the system in terms of the total mass $m_{\mathcal{S}}$ of all the elements of the system inside $\mathcal{S}$, expressed as

$$
p_{\mathcal{S}} \triangleq \grave{\rho}_{\mathcal{S}} A_{\mathcal{S}}=m_{\mathcal{S}}\left(\frac{A_{\mathcal{S}}}{V_{\mathcal{S}}}\right)
$$

The surface area to volume ratio $\left(A_{\mathcal{S}} / V_{\mathcal{S}}\right)$ is well established in the applied sciences as a key determinant of the degree of interactivity between a closed system and its immediate environment. For instance, in cellular biology, the quantity of vital materials needed by a living cell for sustenance depends on the amount of biological matter present in the cell, which depends on its volume. However, the quantity of vital materials that can be exchanged between the cell and its environment depends on the surface area of the cell. If the volume of the cell grows so large that the rate of exchange of vital materials through its surface is insufficient to support its required metabolic rate, then the cell eventually dies. The metabolic rates of living cells thus depend directly on their surface area to volume ratios. Similar logic holds true for the rate at which chemical reactions occur, whereby reactions tend to be more rapid in bodies whose surface area to volume ratio is high than in bodies for which the ratio is low.

In photodynamics, the importance of compactness, and hence of the surface area to volume ratio, lies above all in its role as a key determinant of the potential energy induced in a body by its displacement from equilibrium within its host system, and the restoring (gravitational) force that is produced as a consequence (see Sec. IX B).

\section{G. Rank hierarchy of clusters}

Suppose that a set of $N_{0}$ cells constitutes a first-order cluster $C_{1}$, a set of $N_{1}$ first-order clusters constitutes a second-order cluster $C_{2}$, a set of $N_{2}$ second-order clusters constitutes a third-order cluster $C_{3}$, and so on. If $C_{0}$ is the set of all cells in $C_{1}$, then the extant photino density of any cluster $C_{z}$ is given by

$$
\rho\left(C_{z}\right)=\frac{\sum_{i=1}^{N_{z-1}} H_{A, i}\left(C_{z-1}\right)}{\sum_{i=1}^{N_{z-1}} V_{A, i}\left(C_{z-1}\right)},
$$

where $z \in\{1,2,3, \ldots\}$ is defined as the rank of a cluster within a multi-level hierarchy of nested clusters. Moreover, in Eq. (32), each element $i$ represents one of $N_{z-1}$ proper elements of the cluster $C_{z}$, where a proper element is defined as a unitary cell or cluster of any rank $(z-1)$ that is a direct component of $C_{z}$. To be clear, this statement means that the subelements that lie inside the body of a direct component of $C_{z}$ are not counted as proper elements of $C_{z}$. Meanwhile, from the perspective of the system $C_{z}$, every proper element in it is a distinct unitary body at par with every other proper element. Hence, no proper element of $C_{z}$ has a preferred status, irrespective of its magnitude, relative to any of the other proper elements of the system - except in respect to its density relative to that of the other proper elements.

In addition, if $\bar{\rho}_{\odot, i}$ is the average density of the ambient medium (denoted by $\odot$ ) surrounding a proper element $i$ within a system $C_{z}$, then based on Eq. (28) all proper elements of $C_{z}$ are in equilibrium within the host system if and only if $\left(\rho_{i}-\bar{\rho}_{\odot, i}\right) \simeq 0$ for all $i$. Furthermore, given that $C_{z}$ is itself a proper element within a higher-order system $C_{z+1}$, it follows that $C_{z}$ is in equilibrium within $C_{z+1}$ if and only if its density $\rho\left(C_{z}\right)$ is as close as possible to the average density of its ambient medium $\bar{\rho}_{\odot}\left(C_{z}\right)$, expressed as

$$
\rho\left(C_{z}\right)-\bar{\rho}_{\odot}\left(C_{z}\right) \simeq 0
$$


Given the autonomic propensity of cells to cluster in Gaussian order of cell densities, at the smallest scale, HDT cells come together in characteristic order to form multicell $C_{1}$ clusters as nucleons (i.e., protons and neutrons) and other species of high-density particles. (Note that this model admits the possibility that HDT cells first cluster in characteristic order to form intermediate sub- $C_{1}$ clusters as quarks, which then group together in nearest-density order to form $C_{1}$ clusters as nucleons. See Sec. VII B).

The universality of autonomic behavior dictates that nucleons must, in turn, cluster in (approximately) concentric Gaussian density order to form $C_{2}$ clusters as atomic nuclei. LDT cells (i.e., electrons) then cluster in approximately Gaussian density order around atomic nuclei to form $C_{3}$ clusters as complete atoms. The atoms then cluster in nearest-density order to form $C_{4}$ clusters as molecules, while any gaps that separate individual atoms within a molecule are occupied by LDI cells, arranged always in nearest-density order.

Molecules of like density then cluster locally to form distinct substances at the macroscopic scale. Moreover, any gap separating two nearby molecules must be occupied by LDI cells arranged in nearest-density order, whereby the density of the intervening LDI cells decreases with radial distance away from each molecule until a point of lowest density is reached. The physical boundary between two $C_{4}$ systems is thus defined by the region composed of the lowest-density LDI cells located in the gap separating the two molecules. A complete $C_{4}$ system is therefore defined as the combination of a molecule and the LDI cells arranged around it in radially decreasing nearest density order, up to the boundary that separates the $C_{4}$ system from each of its neighboring systems.

In the framework of the present model, ten rank classifications describe the complete hierarchy of nested clusters, ranging from the subatomic scale to the universe as a whole, whereby $z \in[1,10]$. As noted, individual cells $C_{0}$ and clusters $C_{1}$ to $C_{4}$ all describe microscopic particles. Clusters of higher rank (i.e., $C_{5}$ to $C_{10}$ ) describe celestial bodies and higher order systems, and are discussed later as part of the broader subjects of gravitation and cosmology in Sec. IX A.

Because every cluster, regardless of rank, is composed ultimately of individual cells, the behavior of a cluster reflects the collective behavior of its constituent cells, whereby there exists a self-similarity in the autonomic behavior of clusters that transcends scale. Clusters of every rank therefore substantially mimic the autonomic behavior of individual cells, albeit at speeds that diminish with rank because the number of active elements, and hence the number of autonomic processes entailed, is much greater in clusters of higher rank than in clusters of lower rank.

\section{FOUNDATIONAL MECHANICS}

\section{A. Wave function}

Whereas the photino density $\rho$ describes the total photino content $H$ of a cell per unit volume $V$, the logic of the present model dictates that the density distribution inside any given cell must be anisotropic. Let $s$ be an imaginary subspace of fixed volume $\mathcal{V}$ within a cell such that for a cell composed of $\mathcal{N}$ contiguous subspaces, $V_{A}=\sum_{s=1}^{\mathcal{N}} \mathcal{V}_{s}$. The internal density $\tilde{\rho}$ of a subspace $s$ can then be defined as $\tilde{\rho}_{s}=H_{s} / \mathcal{V}_{s}$, where $H_{s}$ is the photino content of $s$.

Given that cells possess elasticity and consist entirely of photinos, it follows that elasticity must be an innate property of the photinos of which the cells are composed. Therefore, every photino in a host cell seeks a state of elastic equilibrium and is subject to the laws of elasticity. The logic of nearest-density order in respect to the distribution of cells within a system is thus stipulated to extend also to the distribution of photinos inside a cell.

Consequently, photinos inside any given cell $i$ cluster such that the $\tilde{\rho}_{\mathcal{s}}$ values of subspaces located on the boundary of $i$ with a neighboring cell $j$ are made proportional to $\rho_{j}$. (Likewise, the $\tilde{\rho}_{\mathcal{s}}$ values of subspaces inside the neighbor $j$ that are located on its boundary with cell $i$ are made proportional to $\rho_{i}$.) The density distribution of subspaces located on the boundary surface of a cell therefore mirrors the ever-present anisotropy in the density of the medium surrounding the cell, as described previously in Sec. III E. Meanwhile, as per Eq. (33), the density of each subspace in the region separating the boundary surface of cell $i$ from its center of mass $c_{m}$ must be as close as possible to the average density of its neighboring subspaces (which can equivalently be stated symbolically as $\nabla^{2} \tilde{\rho}_{s} \simeq 0$ ). This internal density distribution implies that photino density progressively decreases inward from points on the boundary surface of a cell, reaching its lowest level at $c_{m}$; which further implies that the anisotropic density distribution of the subspaces in its boundary surface is also projected onto the subspaces in the interior of the cell.

This induced anisotropy in the internal photino density distribution of a cell inevitably produces a distinct polarity, which is defined by the axis of symmetry of all $\tilde{\rho}_{s}$ values that together describe the density distribution inside the cell (see Fig. 6). The polar chord of a cell thus passes through its center of mass, while the southward direction of polarity is (arbitrarily) defined as the direction pointing to the higher-density end of the polar chord.

The photino flux density at a point $x$ on the polar chord of a cell is defined herein as the photino density of an imaginary cylindrical slice of the cell, where the cross section of the cylinder lies on a plane orthogonal to the polar chord. The height of the cylinder is $\mathrm{d} x$ and its cross- 


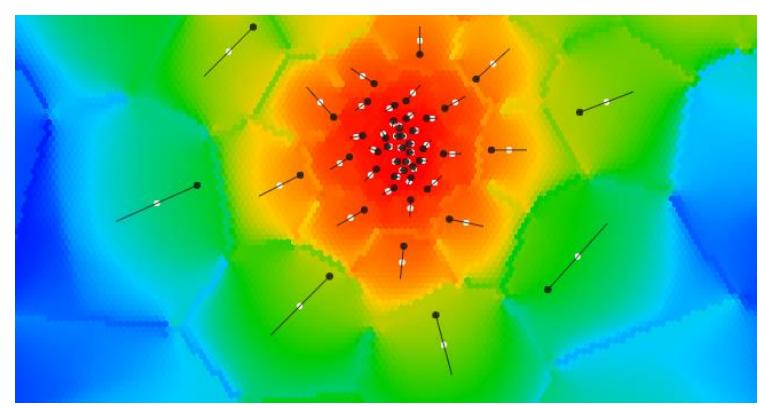

FIG. 6. Cell polarity and internal photino density. The colors denote the grades of photino density of the subspaces within each cell. Red: highest density. Yellow: high-tointermediate density. Green: intermediate-to-low density. Blue: lowest density. (Center of mass of each cell is shown as a white dot.)

sectional area is $Z=\pi R^{2}$, where $R$ is the radius of the cross section (see Fig. 7). Because the density distribution inside a cell reflects the ever-present variations in the densities of the neighboring cells, the photino flux density varies from point to point along the polar chord of the cell. It is greatest at the boundary of a cell with its highestdensity neighbor and decreases progressively along the polar chord until reaching the center of mass of the cell, where the photino flux density is minimal. It then increases progressively along the polar chord until reaching the boundary separating the cell from its lowestdensity neighbor.

The density distribution pattern inside a cell thus approximates a stationary cosine waveform, similar to the distribution of air density in a standing sound wave that forms in a pipe when both ends of the pipe are open to the atmosphere. The standing sound wave has a stationary antinode of maximum amplitude at each of the open ends of the pipe. The fundamental mode of the wave is then defined by a single stationary node of minimum amplitude, located halfway between the two antinodes. If the length of the pipe is $L$, then the wavelength of the standing sound wave in its fundamental mode is given by $\lambda_{1}=2 L$. The next standing wave pattern that satisfies the conditions at the two open ends occurs when $\lambda_{2}=L$, whereby there is one more node and one more antinode within $L$ than in the fundamental mode. In general, the wavelength of a standing sound wave in an open-ended pipe of length $L$ is given by $\lambda_{n}=2 L / n$, where $n \in$ $\{1,2,3, \ldots\}$ denotes the mode of the wave.

In the analogous case of a stationary photino wave within a cell, the photino flux density corresponding to a point $x$ on the polar axis of the cell defines the amplitude $\mathbb{A}$ of the stationary photino wave at $x$, described by $\mathbb{A}_{x}=$ $\left(n_{z} h / Z \mathrm{~d} x\right)_{x}$, where $n_{z}$ is the number of photinos concentrated in the cylindrical slice of volume $Z \mathrm{~d} x$. By analogy to a standing sound wave in its fundamental mode, the two antinodes of maximum amplitude

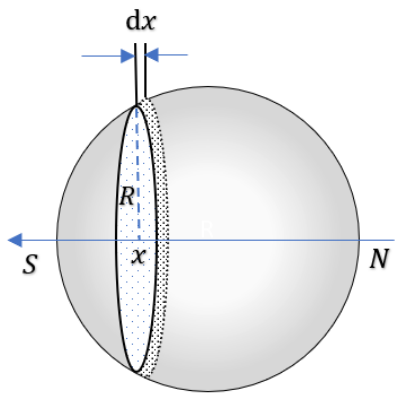

FIG. 7. Amplitude of stationary photino wave at point $x$ on polar chord of cell.

correspond to the two ends of the polar chord of the cell, while a single node of minimum amplitude corresponds to the center of mass of the cell. A stationary photino wave thus (theoretically) describes the approximate photino density distribution inside a cell, while the axis of symmetry of a stationary photino wave represents an alternative description of the polar axis of the cell.

The general form of a cosine wave is given by $\Psi(x, t)=\mathbb{A}_{x} \cos (k x-\omega t)$, where $k$ is the wave number, $\omega$ is the angular frequency, and the function varies with time $t$. However, because the stationary photino wave of a cell is static everywhere, it has no time dependence, whereby its wave function reduces to that in the stationary case $\Psi(x, t=0)=\psi(x)$. Moreover, given that $\psi(x)$ is an approximate representation of the wave function of a stationary photino wave, the reduced wave function can be expressed as $\psi(x) \approx \mathbb{A}_{x} \cos \tilde{k} x$, where $\tilde{k}$ denotes the wave number of the stationary photino wave of a cell. If $L$ is the length of the polar chord of a cell in its ground state, then $x$ represents a point on the polar axis such that $x \in[0, L]$, where $x=0$ denotes the higherdensity (southern) end of the polar chord of the cell and $x=L$ denotes its lower-density (northern) end. The wavelength of a stationary photino wave in its fundamental mode $\lambda_{1}$ (corresponding to the ground state of the cell) is therefore given by $\lambda_{1}=2 L$, while that of a stationary photino wave with $n$ nodes is given by

$$
\lambda_{n}=\frac{2 L}{n} .
$$

The wave number of the stationary photino wave in mode $n$ is then $\tilde{k}_{n}=2 \pi / \lambda_{n}$, whereby the general wave function $\psi_{n}(x)$ can be expressed as

$$
\psi_{n}(x) \approx \mathbb{A}_{x} \cos \left(\frac{2 \pi x}{\lambda_{n}}\right) \approx \mathbb{A}_{x} \cos \left(\frac{n \pi x}{L}\right) .
$$


In the context of photodynamics, polarity is a universal feature that exists in cells as well as in all multicell and higher-order clusters. The polar axis of a rank-1 cluster, such as a nucleon, is the line described by the axis of symmetry of all of the $\rho$ values associated with the cells distributed within the cluster. Because autonomic compulsions enforce the same pattern in the density distribution of their constituent elements, nuclear clusters, complete atoms and higher-order clusters exhibit similar intrinsic polarity.

While their (roughly) concentric spatial distribution requires the densities of the elements of a cluster to decrease radially in each direction from the core of the cluster, quantitative individuality imposes an additional constraint. Elements that are equidistant from the core of the cluster but on diametrically opposite sides along any arbitrary axis that passes through the core must differ in density at least marginally. Consequently, there exists a well-defined, albeit proportionately minor, asymmetry in the surface density of any arbitrary spherical surface centered on the core of a cluster. A cluster whose elements are (largely) in equilibrium thus possesses not only northsouth polarity but also azimuthal polarity, where the latter is (arbitrarily) defined as running from east to west along an azimuthal axis. Surface elements thus increase in density progressively from the northern pole of the surface to its southern pole and decrease in density progressively from the azimuth (analogously, the 'prime meridian') of the surface to its anti-azimuth (analogously, the 'antimeridian' at $180^{\circ}$ longitude).

\section{B. Phase transition}

As noted, extreme compression causes the lower elastic limit of a cell to be breached, given as $V_{A}<V_{R}$, while extreme decompression causes the upper elastic limit of a cell to be breached, given as $V_{A}>V_{P}$. Either event triggers a phase transition, whereby the state of the cell changes spontaneously to one of absolute equilibrium such that $U_{V}=0$.

The phase transition of a cell entails two primary actions, namely the resetting of its $V_{P}$ and $\rho_{R}$ values. Moreover, the equality given by Eq. (12) dictates that a change in $V_{P}$ must be balanced by commensurate changes in its arguments $V_{R}, H_{A}$ and $H_{R}$. However, because $H_{A}$ changes dynamically in nonextreme conditions, only $V_{R}$ and $H_{R}$ change because of a phase transition, where the latter may further trigger a change in $H_{G}$. Consequently, in addition to the two primary actions of resetting the $V_{P}$ and $\rho_{R}$ values of a cell, a phase transition further entails the resetting of the three quantities $V_{R}, H_{R}$ and $H_{G}$ to restore the identity demanded by Eq. (12). The following five-step process describes the mechanism of a phase transition.
Step 1. Reset the par value of the cell to a new value $V_{P}^{\prime}$ such that $V_{P}^{\prime}=V_{A}$.

Step 2. Reset the rudimental volume of the cell to a new value $V_{R}^{\prime}$ such that

$$
V_{R}^{\prime}=V_{R}\left(\frac{V_{A}}{V_{P}}\right)
$$

Rationale: Replacing $V_{P}$ with $V_{P}^{\prime}$ and $V_{R}$ with $V_{R}^{\prime}$ in Eq. (12) and substituting $V_{A}$ for $V_{P}^{\prime}$ in the modified Eq. (12) gives $V_{A}=V_{R}^{\prime}\left[1+(\alpha / 2)\left(\left(H_{A} / H_{R}\right)-1\right)\right]$. Also from Eq. (12), we have $V_{P} / V_{R}=\left[1+(\alpha / 2)\left(\left(H_{A} / H_{R}\right)-1\right)\right]$. Replacing the term $\left[1+(\alpha / 2)\left(\left(H_{A} / H_{R}\right)-1\right)\right]$ with $V_{P} / V_{R}$ in the previous equation yields $V_{A}=V_{R}^{\prime}\left(V_{P} / V_{R}\right)$, of which Eq. (36) is an alternative expression.

Step 3. Reset the rudimental density of the cell to a new value $\rho_{R}^{\prime}$ such that $\rho_{R}^{\prime}=\rho_{A}$.

Step 4. Reset the rudimental photino content of the cell to a new value $H_{R}^{\prime}$ such that

$$
H_{R}^{\prime}=H_{A}\left(\frac{V_{R}^{\prime}}{V_{A}}\right)
$$

Rationale: The identity $\rho_{R}^{\prime}=\rho_{A}$ can be stated alternatively as $H_{R}^{\prime} / V_{R}^{\prime}=H_{A} / V_{A}$, of which Eq. (37) is an alternative expression. Moreover, because $H_{R}^{\prime}$ is the new value of the rudimental photino content of the cell, the new value of the rudimental photino count $n_{R}^{\prime}$ of the cell becomes $n_{R}^{\prime}=H_{R}^{\prime} / h$, which renders $n_{R}^{\prime}$ a mathematically continuous quantity.

Step 5. Reset the ground-state photino content of the cell to a new value $H_{G}^{\prime}$, where $H_{G}^{\prime}=n_{G}^{\prime} h$, by resetting the ground-state photino count of the cell to a new value $n_{G}^{\prime}$ such that

$$
n_{G}^{\prime}= \begin{cases}\left\lfloor n_{R}^{\prime}\right\rfloor & \text { if }\left|n_{R}^{\prime}-\left\lfloor n_{R}^{\prime}\right\rfloor\right|<\left|n_{R}^{\prime}-\left\lceil n_{R}^{\prime}\right\rceil\right| \\ \left\lceil n_{R}^{\prime}\right\rceil & \text { if }\left|n_{R}^{\prime}-\left\lfloor n_{R}^{\prime}\right\rfloor\right| \geq\left|n_{R}^{\prime}-\left\lceil n_{R}^{\prime}\right\rceil\right| .\end{cases}
$$

Rationale: Energy neutrality (i.e., $\eta=0$ ) is ordinarily an unattainable state for a cell because of the fundamentally divergent mathematical properties of the two associated quantities, namely $n_{R} \in \mathbb{R}$ and $n_{G} \in \mathbb{N}^{+}$. Equation (38) is therefore an expression of the physical limitation that the (discrete) ground-state photino count of a cell can equal only the integer part of the (continuous) rudimental photino count of the cell.

Given the dominant role of density in determining the physical behavior of a cell, any change in the rudimental density of a cell is transformational. It alters fundamentally the physical character of the cell as a 
particle and this altered character then remains a permanent feature of the cell in all nonextreme conditions.

Note that the mechanism of phase transition described above is the same whether it is the lower or the upper elastic limit of a cell that is breached. However, the physical effect in either case is the converse of the other. A phase transition due to breaching the lower elastic limit transforms a cell into a heavier particle by increasing its rudimental density, while a reverse phase transition due to breaching the upper elastic limit transforms the cell into a lighter particle by reducing its rudimental density.

\section{Energy transmission process}

In photodynamics, the transmission of energy as radiation is described simply as the conduction of photinos from an emitting cell to a receiving neighbor under the compulsions induced by the autonomic principle $U_{V} \rightrightarrows 0$. A cell can be described as being energized if its extant photino count $n_{A}$ exceeds its ground-state photino count $n_{G}$, while the difference $\left(n_{A}-n_{G}\right)$ defines the number of unbound photinos resident in the cell.

Suppose that a cell in its ground state becomes energized such that its $H_{A}$ is increased, while the spatial constraints imposed by its local environment inhibit an increase in its $V_{A}$ commensurate with the increase, per Eq. (12), in its $V_{P}$. In that case, $V_{P}>V_{A}$ and the cell acquires potential energy $U_{V}>0$ in accordance with Eq. (13), while the autonomic principle dictates that $U_{V} \rightrightarrows 0$. Pursuant to Eq. (19), the $U_{V}$ of the cell can then be reduced if and only if its $H_{A}$ (and hence $n_{A}$ ) decreases through the emission of one or more unbound photinos. Furthermore, given the spatial contiguity of cells in a system, a photino emitted by a cell must be absorbed by a neighboring cell. Meanwhile, the unit-action limit dictates that a cell can emit no more than one photino per time step.

The process of cell-to-cell photino transmission can be then described within the general framework of a chain of three cells $i, j$ and $k$ in which cell $i$ is energized, and cells $j$ and $k$ are initially in their ground states. If cell $j$ receives successive photinos emitted by its neighbor $i$ at frequency $v_{i n}$, then the wavelength of the incident photino wave is $\lambda_{\text {in }}=c / v_{\text {in }}$, and the corresponding wave number is $k_{\text {in }}=2 \pi / \lambda_{\text {in }}$. In addition, the autonomic principle dictates that the onward transmission of photinos from cell $j$ to cell $k$ must reduce the total elastic potential energy of the cell pair $\{j, k\}$, implying an entropic condition given by the relation

$$
\frac{\left(U_{V, j}-U_{V, j}^{\prime}\right)}{\left(U_{V, k}^{\prime}-U_{V, k}\right)}>1
$$

where $U_{V, j}$ and $U_{V, k}$ are the elastic potential energies of cells $j$ and $k$, respectively, prior to the transmission of photinos and $U_{V, j}^{\prime}$ and $U_{V, k}^{\prime}$ are the respective values subsequent to the transmission.

In the context of photodynamics, resonance occurs between an incident photino wave and a receiving cell $j$ when $k_{i n}$ is equal to the extant harmonic wave number $\tilde{k}_{n, j}$ of the stationary photino wave of cell $j$. According to the present model, the onward transmission of photinos (i.e., from cell $j$ to $k$ ) is regulated not by a single unique mechanism but rather by two alternative mechanisms, where the absence of resonance triggers one mechanism, while the presence of resonance triggers the other. The mechanism of photino transmission in the absence of resonance can be characterized as pass-through conduction, while the mechanism in the presence of resonance can be characterized as photonic transmission, as follows.

Pass-through conduction. Suppose that cell $j$ receives successive photinos from cell $i$ at incident frequency $v_{i n}$, and the incident photino waves do not resonate with cell $j$. In that case, the present model stipulates that cell $j$ must transmit its resident unbound photinos onward to cell $k$ at the same frequency as the incident frequency $v_{i n}$, if the entropic condition is satisfied. (To be clear, no photinos can be transmitted by cell $j$ during periods in which the entropic condition is not satisfied.)

The transmission of photinos via pass-through conduction therefore occurs without the frequency of the transmitted photino waves being altered in the process. Pass-through conduction is the essential mechanism by which photinos are transmitted from cell to cell in a medium, and thereby constitutes the mechanism for the propagation of radiation energy across spatial distances.

Photonic transmission. Suppose that cell $j$ is in its ground state whereby $n=1$, and incident photino waves arriving from cell $i$ resonate with cell $j$ such that $k_{i n}=\tilde{k}_{1, j}$. The present model stipulates that the effect of resonance is to conditionally inhibit the emission of photinos by cell $j$. Successive photinos received by cell $j$ from cell $i$ are thus retained in $j$ and accumulate as unbound photinos until cell $j$ has reached a higher harmonic state such that the mode $n$ of its stationary photino wave is increased by an integer value.

According to this model, if $n>1$ and the entropic condition given by Eq. (39) is satisfied, then cell $j$ must emit photinos successively at its resonant frequency $v_{\text {res }}$ to cell $k$ until cell $j$ has descended to a lower harmonic state, where

$$
v_{\text {res }}=\frac{c}{2 \pi} \tilde{k}_{n, j} .
$$


This mechanism further implies that if $n>1$ but the entropic condition is not satisfied while $k_{i n}=\tilde{k}_{n, j}$, then cell $j$ must continue to absorb and retain the photinos received from cell $i$ via successive photino waves until cell $j$ has reached the next higher harmonic state. In principle, therefore, the mode $n$ of the stationary photino wave of cell $j$ can increase to any integer value as long as the entropic condition remains unsatisfied. In reality, however, the values of $n$ tend to be restricted to small integers by the autonomic compulsions that are everpresent in physical systems.

Suppose that cell $j$ continues to absorb photinos received from cell $i$ via successive photino waves such that the mode of its stationary photino wave is raised from the initial value of $n=1$ to, say, $n=5$. If at this point the entropic condition is satisfied, then cell $j$ must emit photinos successively to reduce its potential energy in accordance with Eq. (19) and thereby descend to a lower harmonic state. However, given that the mode of the stationary photino wave of cell $j$ is now $n=5$, the value of $n$ can potentially decrease by one level to $n=4$, or by two levels to $n=3$, or by three levels to $n=2$, or by four levels to $n=1$. The number of levels by which the value of $n$ decreases in any given case is then determined as follows. Let $\mathcal{C}$ be a set containing all possible levels $q$ by which $n$ may decrease in value. The autonomic principle dictates that $n$ must decrease by an optimal value $\mathcal{Q}$ such that the transmission of photinos from cell $j$ to cell $k$ produces the largest reduction in the total elastic potential energy of the cell pair $\{j, k\}$, expressed symbolically as

$$
\mathcal{Q}=\underset{q \in \mathcal{C}}{\arg \max }\left(\frac{U_{V, j}(q)-U_{V, j}^{\prime}(q)}{U_{V, k}^{\prime}(q)-U_{V, k}(q)}\right) .
$$

Photonic transmission thus entails ensembles of photinos being absorbed and emitted (at resonant frequencies) by cells as distinct unitary objects. In the context of photodynamics, such an ensemble of photinos when absorbed or emitted by a cell as a unitary object represents a photon. The energy $E$ of a photon is then described by the energy equivalent to the total number of photinos $n$ transmitted per unit time by the photon, which, according to Eqs. (5) and (7) is given as $E=n h v_{0}=h v$, where $v=v_{\text {res }}$ defines the frequency of the photon.

Photonic transmissions are central to the mechanism underlying the Compton effect described below, as well as the line spectra associated with the chemical elements (see Sec. VIII B).

\section{Compton effect}

Photodynamics describes the Compton effect [29] as a feature induced by the autonomic principle. When an incident photon transmits photinos that are absorbed by a cell, the $V_{P}$ of the cell must increase in accordance with Eq. (12). If environmental constraints inhibit a corresponding increase in its $V_{A}$, then $V_{P}>V_{A}$ and the cell acquires potential energy $U_{V}>0$ according to Eq. (13), while the autonomic principle dictates that the cell must then minimize $U_{V}$. A reduction in $U_{V}$ requires either the onward transmission of photinos from the cell to a neighbor via photon emission or the conversion of $U_{V}$ into kinetic energy $K$, thus inducing motion in the cell; while both photon emission and cell motion may ensue if the prevailing local conditions are conducive.

As stated, the stationary photino wave of a cell is a representation of the internal distribution of photino density relative to points along the polar chord of the cell. The fundamental harmonic $(n=1)$ of a stationary photino wave describes the photino density distribution inside a cell when the cell is in its ground state, whereby the photino count of the cell is $n_{G}$. The second harmonic $(n=2)$ then describes the photino density distribution inside the cell when it has absorbed the additional $n_{v}$ photinos transmitted to it by an incident photon at frequency $v$, whereby the extant photino count of the cell becomes $n_{G}+n_{v}$

The wavelength of a stationary photino wave is given by Eq. (34) as $\lambda_{n}=2 L / n$, which implies a corresponding frequency $v_{n}=c / \lambda_{n}=c n / 2 L$. The implied fundamental harmonic frequency of a stationary photino wave is therefore $v_{1}=c / 2 L$ and the implied second harmonic frequency is $v_{2}=c / L$, whereby $v_{2}=2 v_{1}$. Multiplying both sides of this equation by $1 / v_{0}$ gives $v_{2} / v_{0}=2\left(v_{1} / v_{0}\right)$, which, based on Eq. (7), yields the relationship $n_{2}=2 n_{1}$, where $n_{1}$ and $n_{2}$ denote the number of photinos present in the stationary photino wave in modes $n=1$ and $n=2$, respectively.

Given that the fundamental harmonic of a stationary photino wave corresponds to a cell in its ground state, it follows that $n_{1}=n_{G}$, and because the second harmonic corresponds to the state of the cell after it has absorbed the $n_{v}$ photinos received via an incident photon, it follows that $n_{2}=n_{G}+n_{v}$. Substituting accordingly for $n_{1}$ and $n_{2}$ in the above-derived relationship $n_{2}=2 n_{1}$ gives $n_{v}=n_{G}$.

Let $\lambda$ be the wavelength of a photon incident upon a cell and $\lambda^{\prime}$ be the wavelength of the photon emitted by the cell subsequent to its absorption of the photinos received via the incident photon. The difference between the wavelengths of the emitted and incident photons is then given by $\Delta \lambda=\lambda^{\prime}-\lambda$. Multiplying the right-hand side of this equation by $h / h$ gives $\Delta \lambda=\left(h \lambda^{\prime} / h-h \lambda / h\right)=$ $\left(h c / h v^{\prime}-h c / h v\right)$, which, pursuant to Eq. (7), can be stated as

$$
\Delta \lambda=\left(\frac{h c}{n_{v^{\prime}} h v_{0}}-\frac{h c}{n_{v} h v_{0}}\right),
$$


where $n_{v^{\prime}}$ is the number of photinos transmitted from the cell via the photon emitted at frequency $v^{\prime}$. As stated, when $n=2$, the photino count of the cell is $n_{G}+n_{v}$ before the emission of a photon, while its photino count after the transmission of $n_{v^{\prime}}$ photinos via the emitted photon can be no less than $n_{G}$, whereby $0 \leq n_{v^{\prime}} \leq n_{v}$. Furthermore, because $n_{v}=n_{G}$ when $n=2$, this relationship can be expressed equivalently as $n_{v^{\prime}}=n_{G} \varpi$, where $0 \leq \varpi \leq 1$. Substituting accordingly in Eq. (42) gives $\quad \Delta \lambda=\left(h c / \varpi n_{G} h v_{0}\right)-\left(h c / n_{G} h v_{0}\right)$, which reduces to $\Delta \lambda=\left(h c / n_{G} h v_{0}\right)((1 / \varpi)-1)$. Based on Eq. (5), this equation becomes $\Delta \lambda=\left(h c / E_{G}\right)((1 / \varpi)-1)$, where $E_{G}$ is the ground-state energy content of the cell. This relationship can be expressed alternatively as $\left(\lambda^{\prime} / h c\right)-(\lambda / h c)=\left(1 / E_{G}\right)((1 / \varpi)-1)$, which can be written as $\left(1 / E^{\prime}\right)-(1 / E)=\left(1 / E_{G}\right)((1 / \varpi)-1)$, where $E$ is the energy transported by the incident photon and $E^{\prime}$ is the energy transported by the emitted photon. Supposing that the cell is an electron located in an atom and defining $\varpi$ such that $\varpi \triangleq\left(E^{\prime} / E\right)$, the previous equation becomes

$$
\frac{1}{E^{\prime}}-\frac{1}{E}=\left(\frac{1}{E_{G}}\right)\left[\frac{K^{*}}{E^{\prime}}\right]
$$

where $E=E^{\prime}+K^{*}$ and $K^{*}=K+\varphi$, while $K$ is the kinetic energy of the electron and $\varphi$ is the photoelectric work function specific to its neighborhood, representing the (ionization) energy needed to dislodge the electron from its host atom [24]. The physical mechanism underlying Eq. (43) entails the $n_{v}$ photinos received via the incident photon being added to the ground-state photino count $n_{G}$ of the electron. As per Eq. (12), the $V_{P}$ of the electron increases as a consequence of its increased photino content. If the neighboring particles in and around the host atom inhibit a corresponding increase in its $V_{A}$, then $V_{P}>V_{A}$, which induces potential energy $U_{V}>0$ in the electron in accordance with Eq. (13). The induced potential energy of the electron then dissipates as $U_{V} \rightrightarrows 0$, partly through the transmission of the energy $E^{\prime}$ carried by the emitted photon and partly through its conversion into $\varphi$ plus kinetic energy $K$. The latter produces motion, causing the electron to escape from its host atom.

Let $\theta$ be the scattering angle formed by the trajectory of the emitted photon relative to the axis of the trajectory of the incident photon. According to the present model, the scattering angle $\theta$ varies such that $\cos \theta=\left(E^{\prime}-K^{*}\right) / E^{\prime}$ over the range $0 \leq \theta \leq \pi$. Equation (43) can then be restated accordingly by replacing $K^{*} / E^{\prime}$ with $[1-\cos \theta]$ to give the well-known formula of conventional atomic theory for Compton scattering, written as

$$
\frac{1}{E^{\prime}}-\frac{1}{E}=\frac{1}{E_{G}}[1-\cos \theta]
$$

This equation relates the energy of a scattered photon $E^{\prime}$ to the energy of the incident photon $E$ and the scattering angle $\theta$. Experimental evidence supporting the veracity of this formula is well documented.

Equation (44) can be expressed for the general case of a cell as $\left(\lambda^{\prime} / h c\right)-(\lambda / h c)=\left(1 / m_{G} c^{2}\right)[1-\cos \theta]$, where $m_{G}$ is the ground-state rest mass of the cell. Multiplying both sides of this equation by $h c$ yields the Compton shift formula

$$
\Delta \lambda=\left(\frac{h}{m_{G} c}\right)[1-\cos \theta] .
$$

Conventionally, the quantity $h / m_{G} c$ is described as the Compton wavelength $\lambda_{C}$ associated with an elementary particle. In its fundamental mode, the wavelength of the stationary photino wave associated with a cell can be described equivalently by the relationships in the chain of equalities

$$
\lambda_{1}=\frac{h c}{h v_{1}}=\frac{h c}{m_{G} c^{2}}=\frac{h}{m_{G} c}=\lambda_{C} .
$$

This equation asserts that the Compton wavelength of a cell is a measure of the wavelength, in the fundamental mode, of the stationary photino wave associated with the cell in its ground state.

As a single-cell particle, the Compton wavelength of an electron can be readily determined to be $\lambda_{C(\mathrm{e})} \approx$ $2.426 \times 10^{-12} \mathrm{~m}$ by replacing $m_{G}$ in the formula $\lambda_{C}=$ $\left(h / m_{G} c\right)$ with the rest mass $m_{\mathrm{e}}$ of an electron. Given that a proton is a multi-cell cluster, strictly speaking, the absorption and emission of a photon by a proton occurs only in one of its constituent cells, which can be referred to as the interacting cell. Because the interacting cell remains bound to its host cluster (i.e., the proton), any kinetic energy induced in the interacting cell by its absorption of photinos received via the incident photon must set the complete cluster, namely the proton as a whole, in motion. Consequently, the rest mass associated with the Compton wavelength of a proton is not that of the interacting cell alone, but rather it is the mass of the proton as a whole. For all practical purposes, therefore, a proton can be treated as a virtual single-cell particle of rest mass $m_{\mathrm{p}}$, whereby its Compton wavelength can be calculated to be $\lambda_{C(\mathrm{p})} \approx 1.321 \times 10^{-15} \mathrm{~m}$ by replacing $m_{G}$ in the formula for $\lambda_{C}$ with the proton mass $m_{\mathrm{p}}$.

By extension, $\lambda_{C}$ can be similarly determined for any atomic-scale particle by replacing $m_{G}$ in the formula for $\lambda_{C}$ with the rest mass of that particle. Experimental evidence indicates that an electron that remains bound to its host atom does not produce an appreciable Compton shift. In agreement with the conventional view, this is because the effective mass of a bound electron is not the 
mass of the electron alone, but rather it is the (far greater) rest mass of the host atom to which the electron is bound.

\section{E. Photoelectric effect and Brownian motion}

From Einstein's description of the photoelectric effect [24], the kinetic energy of an electron ejected from an atom upon absorbing an incident photon is given by

$$
K=h v-\varphi
$$

According to this description, the mechanism of the photoelectric effect entails the transfer of the linear momentum $p(=h / \lambda)$ of an incident photon to the electron, causing the latter to detach from the host atom and move with kinetic energy $K$.

The present model describes the photoelectric effect also as a feature induced by the autonomic principle. If an electron within an atom is in an equilibrium state, then its $V_{A} \simeq V_{P}$, whereby according to Eq. (13), its $U_{V} \simeq 0$. Upon absorbing the photinos transmitted to it via an incident photon, the photino content of the electron increases, whereby the $V_{P}$ of the electron increases correspondingly in accordance with Eq. (12). If the spatial limitations imposed by neighboring electrons in the host atom inhibit a proportional increase in its $V_{A}$, then $V_{P}>V_{A}$ and the electron descends into a nonequilibrium state such that $U_{V}>0$. Pursuant to the autonomic compulsion $U_{V} \rightrightarrows 0$, the potential energy of the electron decreases by conversion into kinetic energy such that $K=U_{V}-\varphi$. Motion is thus induced by the kinetic energy gained, propelling the electron out of its location inside the atom in pursuit of a return to an equilibrium state $U_{V} \simeq 0$. In essence, the photoelectric effect represents a limiting case of the Compton effect in which $U_{V}>\varphi$.

The description of Brownian motion in the present model entails similar reasoning. A particle (e.g., a pollen grain) suspended in a fluid is observed to display erratic motion with trajectories that vary randomly in both direction and magnitude. Einstein's description of this phenomenon [30, 31] attributes the erratic motion to the recoil produced in the particle in response to the buffeting by molecules in the fluid, which are intrinsically in a perpetual state of random motion.

Photodynamics describes Brownian motion as another consequence of the autonomic principle. Photinos transmitted via incident photons are absorbed by the electrons of the exterior atoms in the surface molecules of a particle. The $V_{P}$ of a photon-receiving electron thus increases, while spatial constraints within its host atom inhibit a proportional increase in its $V_{A}$, thus inducing $U_{V}>0$. As $U_{V}$ decreases in accordance with the autonomic principle, it converts into kinetic energy such that $K=U_{V}-\varphi$. However, in this case, unlike in the photoelectric effect, the electron is not dislodged but instead remains bound to its host atom. It is therefore the kinetic energy induced in the (bound) constituent electrons of the particle that generates the impulse for the motion of the particle as a whole. Brownian motion is therefore also a limiting case of the Compton effect, in which $U_{V}<\varphi$. The direction and magnitude at each step in the motion of the particle are determined by the geometric locations of the photon-receiving electrons on the surface of the particle and the number of photinos absorbed. Because any number of photons may reach the particle from any direction, the motion of the particle is typically erratic.

\section{LIGHT, OPTICS, AND THERMODYNAMICS}

Photinos subsist in high concentrations within the HDT and LDT cells that make up material bodies across the universe, and in lower concentrations within the predominantly LDI cells that collectively make up the medium of space spanning the cosmos. Consequently, given that no cell can be devoid of photinos, it follows that the total photino count in the universe must far exceed its total cell count. Every cell therefore typically hosts a great number of photinos, as exemplified by the photino count $n_{\mathrm{e}} \sim 10^{20}$ in the single-cell body of an average electron derived in Eq. (9).

Prior to its emission from a given cell $i$ to a neighbor $j$ in accordance with the entropic process of energy transmission described in Sec. IV C, a photino subsists as one amongst all of the photinos that lie within $i$. The photino enters $j$ during transmission and thereafter remains with all of the other photinos in $j$. This process is repeated when $j$ emits a photino to another neighbor $k$. However, the photino emitted by $j$ and absorbed by $k$ is then almost surely not the photino previously transmitted from $i$ to $j$. It is another photino, which, prior to the transmission of the photino from $i$ to $j$, lay among all other photinos in $j$. The process of energy transmission therefore does not require the displacement of any given photino farther than a single interface separating two cells. Unlike the motion of bodies of matter, light does not traverse space through the physical movement of photinos over large distances. It propagates through a chain of successive conduction actions, where each action entails a (different) photino being tipped across a single interface between two neighboring cells.

Furthermore, in the context of the cell-to-cell conduction of photinos, the diameter of a conducting cell $j$ is defined by the length of the chord connecting the point of entry of a photino into $j$ from $i$ to the point of exit of a photino from $j$ to $k$. 


\section{A. Refractive index of light}

It is common to observe the refraction of light when it enters, say, a slab of glass from air if the angle of incidence of the light deviates from the normal to the surface of the glass. Snell's law describes this effect accurately with the relation $\sin \theta_{2}=\left(\mathfrak{n}_{1} / \mathfrak{n}_{2}\right) \sin \theta_{1}$, where the light transmitted initially in a medium of refractive index $n_{1}$ enters a medium of refractive index $\mathfrak{n}_{2}$, and $\theta_{1}$ and $\theta_{2}$ are the respective angles of incidence and refraction of the light. At the macroscopic level, refraction can thus be understood easily as the geometric alteration in the trajectory of light by a change in the refractive index of the medium. However, at the microscopic level, the mechanism that causes the wavelength of light and its speed to be altered by the refractive index of the medium is more intricate.

In the context of photodynamics, the spatial composition of any medium in which light is transmitted is cellular at an elemental level. As the (contiguous) cells that make up a medium emit unbound photinos to minimize their stored potential energy, each cell approaches its ground state such that $n_{A} \simeq n_{G}$ as the system reaches equilibrium. Meanwhile, the relative differences in the ground-state photino contents of cells located in close proximity to one another in a medium tend to be proportionately minor. For instance, suppose that the $n_{G}$ value of a cell $i$ is roughly $1 / 1000^{\text {th }}$ the $n_{G}$ of an average electron (i.e., $n_{G, i} \sim 10^{17}$ ) while the $n_{G}$ value of a neighboring cell $j$ exceeds that of cell $i$ by $10^{6}$ photinos, whereby $n_{G, j} \sim\left(10^{17}+10^{6}\right)$. It then follows that the relative difference in the photino counts of cells $i$ and $j$, as measured by the ratio $\left|n_{G, j}-n_{G, i}\right| / n_{G, j}$, would have a proportionately minor numerical value of roughly $10^{-11}$. By contrast, the relative differences in the physical volumes of neighboring cells in a medium, as measured by the ratio $\left|V_{A, j}-V_{A, i}\right| / V_{A, j}$, tend typically to be much more pronounced (see Fig. 1).

Consequently, the relative densities of cells located in close proximity to one another in a medium are determined primarily not by the (proportionately minor) differences in their respective photino contents, but rather by the (more pronounced) ever-present differences in their physical volumes. Accordingly, the average volume, and hence the average diameter, of the cells that make up a medium tends to vary inversely with the density of the medium. Light propagates more slowly in a dense medium than in a rare medium because the average diameter of cells is smaller in a dense medium, whereby the photinos transmitted in a dense medium traverse a shorter distance per unit time than those that are transmitted at the same frequency in a rare medium.

By convention, the refractive index $\mathfrak{n}_{\mathfrak{m}}$ of a medium $\mathfrak{m}$ is defined as the ratio of the terminal speed of light $c$ to the speed of its propagation in the medium $c_{\mathfrak{m}}$, stated as $\mathfrak{n}_{\mathfrak{m}}=c / c_{\mathfrak{m}}$. Conventional theory attributes this inverse relationship between $c_{\mathfrak{m}}$ and $n_{\mathfrak{m}}$ to the dynamics of electric fields and associated feedback effects induced by oscillating charges present in a medium [32]. Contrary to conventional theory, photodynamics ascribes the inverse relationship between $\boldsymbol{n}_{\mathfrak{m}}$ and $c_{\mathfrak{m}}$ to the spatial geometry of the medium, as follows.

Let $\bar{r}_{\mathfrak{m}}$ be the average radius of the cells through which light is conducted in a given medium $\mathfrak{m}$. If $g_{\mathfrak{m}}$ is the number of cell diameters per unit distance traversed by the light along its path through the medium, then it follows that $g_{\mathfrak{m}}=1 / 2 \bar{r}_{\mathfrak{m}}$. If $\lambda_{\mathfrak{m}}$ is the wavelength of the light, then we have $k_{\mathrm{m}}=2 \pi / \lambda_{\mathrm{m}}$, where the wave number $k_{\mathrm{m}}$ denotes the number of radians per unit distance. Thus, if $z_{\mathfrak{m}}$ is defined as the number of cell diameters per radian traversed by the light, then $z_{\mathfrak{m}}=g_{\mathfrak{m}} / k_{\mathfrak{m}}=$ $\lambda_{\mathfrak{m}} /\left(2 \pi\left(2 \bar{r}_{\mathfrak{m}}\right)\right)$, which can be written as

$$
2 \bar{r}_{\mathfrak{m}} z_{\mathfrak{m}}=\frac{\lambda_{\mathfrak{m}}}{2 \pi}
$$

Multiplying both sides of this equation by the angular frequency $\omega_{\mathfrak{m}}$ of the light, where $\omega_{\mathfrak{m}}=2 \pi v_{\mathfrak{m}}$ and $v_{\mathfrak{m}}$ is the linear frequency of the light, we have

$$
\left(2 \bar{r}_{\mathfrak{m}} z_{\mathfrak{m}}\right) \omega_{\mathfrak{m}}=\lambda_{\mathfrak{m}} v_{\mathfrak{m}}=c_{\mathfrak{m}} .
$$

As described in Sec. II B, the limiting wavelength $\lambda_{\tau}$ of light entails the (hypothetical) conduction of successive photinos from one cell to the next in each consecutive time step, whereby $\lambda_{\tau}=2 r_{\tau}$, where $r_{\tau}$ is the limiting cell radius. Multiplying both sides of this equation by $v_{\tau}$ gives

$$
2 r_{\tau} v_{\tau}=\lambda_{\tau} v_{\tau}=c
$$

Dividing Eq. (50) by Eq. (49) then gives the refractive index of the medium as

$$
\frac{c}{c_{\mathfrak{m}}}=\mathfrak{n}_{\mathfrak{m}}=\frac{r_{\tau} v_{\tau}}{\left(\bar{r}_{\mathfrak{m}} z_{\mathfrak{m}}\right) \omega_{\mathfrak{m}}}
$$

Given that $r_{\tau}=\lambda_{\tau} / 2$ and from Eq. (48) we have $\bar{r}_{\mathrm{m}} Z_{\mathrm{m}}=\lambda_{\mathrm{m}} / 4 \pi$, Eq. (51) can be written alternatively as $\mathfrak{n}_{\mathfrak{m}}=\left(2 \pi \lambda_{\tau} \nu_{\tau}\right) /\left(\lambda_{\mathfrak{m}} \omega_{\mathfrak{m}}\right)$. This equation can be stated as $n_{\mathfrak{m}}=2 \pi c / \lambda_{\mathfrak{m}} \omega_{\mathfrak{m}}$ or, equivalently, as $\lambda_{\mathfrak{m}}=2 \pi c / \mathfrak{n}_{\mathfrak{m}} \omega_{\mathfrak{m}}$. Because the frequency of light (i.e., the number of photinos transmitted per unit time) is determined solely by the light-emitting source, $\omega_{\mathfrak{m}}$ does not depend on the medium in which the light is transmitted. Therefore, for the general case in which light is transmitted at angular frequency $\omega$, the previous equation can be written as

$$
\lambda_{\mathrm{m}}=\frac{2 \pi c}{\mathrm{n}_{\mathrm{m}} \omega} .
$$


This equation is well-established in conventional theory as an accurate description of the dependence of the wavelength of light on the refractive index of the medium in which the light is transmitted. Experimental evidence supporting the veracity of the formula given by Eq. (52) is well documented.

According to photodynamics, the refraction of light constitutes the principal phenomenon in optics, from which every other optical effect follows.

\section{B. Diffraction}

The present model describes the diffraction of light passing through a narrow aperture as a characteristic feature produced by the spatial geometry of the medium spanning the aperture. The autonomic principle dictates that each cell within the medium spanning the aperture must migrate to its nearest-density location. Hence, because of the high-density material composition of the screen in which the aperture is located, the highest-density cells in the medium are compelled to migrate to points around the edges of the aperture, while all the other cells in the medium are compelled to relocate to their respective nearest-density locations within the aperture.

Cells therefore decrease progressively in density from the edges towards the center of the aperture. This arrangement produces a spatial geometry in which the cell volumes, and hence the cell diameters, increase progressively from the edges towards the center of the aperture. As light passes through the aperture, this effective 'lensing' of the medium shortens the wavelength of the light the most around the edges of the aperture and progressively less so towards its center. Consequently, the distance traversed per unit time by photinos conducted through the center of the aperture exceeds the distance traversed in the same time period by photinos conducted closer to the edges of the aperture. Thus, an arced wavefront, characteristic of diffraction, forms as the light emerges from the aperture.

\section{Temperature and pressure}

Based on the statistical mechanics conventionally associated with molecular motion $[33,34]$, the pressure $P$ exerted by a (monatomic) gas is defined by the thermodynamic equation $P=(2 / 3)(N / V) \bar{K}$, which can be restated as $P V=(2 / 3) N \bar{K}$, where $\bar{K}$ is the average kinetic energy of the $N$ gas molecules contained within a volume $V$. Moreover, the empirical equation of state for gases is described by the relation $P V=N k_{B} T$, where $T$ denotes the temperature and $k_{B} \approx 1.381 \times 10^{-23} \mathrm{~J} \mathrm{~K}^{-1}$ is Boltzmann's constant. Equating the two relationships yields $T=(2 / 3)\left(\bar{K} / k_{B}\right)$. Conventional theory thus describes temperature as a measure of the average kinetic energy of molecules in a system, where translational motion within an otherwise empty volume of space is deemed an intrinsic state of the molecules in the system.

The logic of photodynamics implies a fundamental departure from the paradigm of statistical mechanics. While molecular motion tends to be present in a system, motion is neither an intrinsic state of molecules nor the fundamental source of the mechanical energy of a system. Rather, the ultimate source of the mechanical energy of a system is the potential energy induced by the elastic nonequilibrium of its cellular elements caused by compression. Molecular motion is thus a derivative effect that is produced by the kinetic energy gained as the cellular elements of a system are compelled to reduce their potential energy in pursuit of elastic equilibrium, in accordance with the autonomic principle.

Temperature and pressure are therefore described as variant functions of the elastic potential energy stored in a system. The temperature of a system represents a measure of the average elastic potential energy $\bar{U}_{V}$ of the cellular elements of the system. Substituting $\bar{U}_{V}$ for $\bar{K}$ in the thermodynamic equation for $T$ yields

$$
T=\frac{2}{3} \frac{\bar{U}_{V}}{k_{B}},
$$

where the average elastic potential energy of a system composed of $\widetilde{N}$ cells is given according to Eq. (13) by

$$
\bar{U}_{V}=\frac{v_{0}}{(2 \widetilde{N})} \sum_{i=1}^{\bar{N}} H_{A, i}\left(\left(\frac{V_{P, i}}{V_{A, i}}\right)-1\right)^{2}
$$

Substituting $\bar{U}_{V}$ for $\bar{K}$ and $\widetilde{N}$ for $N$ in the thermodynamic equation for $P$ gives

$$
P=\frac{2}{3}\left(\frac{\widetilde{N}}{V}\right) \bar{U}_{V}
$$

From Eqs. (53) and (54), the temperature of a single cell is described by the case where $\widetilde{N}=1$ as

$$
T=\frac{2}{3} \frac{U_{V}}{k_{B}}=\frac{H_{A} v_{0}}{3 k_{B}}\left(\frac{V_{P}}{V_{A}}-1\right)^{2} .
$$

Similarly, the internal pressure of a single cell is given by the case where $\breve{N}=1$. Thus, from Eqs. (55) and (54) the internal pressure of a single cell can be described by the relationship $P=2 U_{V} / 3 V_{A}=\left(\rho_{A} v_{0} / 3\right)\left(\left(V_{P} / V_{A}\right)-1\right)^{2}$.

If $\kappa_{C}$ is the compression $\left(V_{P} / V_{A}\right)$ of a cell such that $\kappa_{C}$ is held constant, then the temperature of the cell is given by Eq. (56) as $T=\left(H_{A} v_{0} / 3 k_{B}\right)\left(\kappa_{C}-1\right)^{2}$. If a quantity $\gamma$ is defined such that $\gamma \triangleq\left(v_{0} / 3 k_{B}\right)\left(\kappa_{C}-1\right)^{2}$, then $T=\gamma H_{A}$, whereby 


$$
H_{A}=\frac{1}{\gamma} T
$$

Equation (12) can be stated in an alternative form as $V_{P}=V_{R}\left[(\alpha / 2)\left(H_{A} / H_{R}\right)+(1-(\alpha / 2))\right]$. Replacing $H_{A}$ in this equation with the right-hand side of Eq. (57) gives

$$
V_{P}=V_{R}\left(\frac{\alpha}{2} \frac{T}{\gamma H_{R}}+\left(1-\frac{\alpha}{2}\right)\right) .
$$

If $T=0$, then Eq. (58) becomes $V_{P \mid T=0}=V_{R}(1-(\alpha / 2))$, which can be restated as $V_{R}=V_{P \mid T=0} /(1-(\alpha / 2))$. Replacing $V_{R}$ in Eq. (58) with the right-hand side of this equation, following some algebraic rearrangement we get $V_{P}=V_{P \mid T=0}+V_{P \mid T=0}\left[T / \gamma H_{R}((2 / \alpha)-1)\right]$. Defining a quantity $\beta \triangleq 1 /((2 / \alpha)-1)$ reduces the previous equation to

$$
V_{P}=V_{P \mid T=0}\left(1+\beta \frac{T}{\gamma H_{R}}\right)
$$

This relationship describes the hypothetical case in which the temperature of a cell may be reduced to absolute zero. According to Eq. (56), $T=0$ is possible if and only if $V_{P}=V_{A}$, this being because the photino content $H_{A}$ of a cell cannot be zero. However, given that $\left(V_{P} / V_{A}\right)=\kappa_{C}$, it follows that if $T=0$ then $\kappa_{C}=1$, whereby $\gamma=0$ and Eq. (57) yields the indeterminate value $H_{A}=0 / 0$, which is patently absurd. Therefore, it must be strictly true that $V_{P} \neq V_{A}$, whereby $T=0$ is a prohibited state for a cell and hence for any physical system in nonextreme conditions.

By definition, the $V_{A}$ of a cell can be no less than its $V_{R}$ and no greater than its $V_{P}$, while $V_{P}=V_{A}$ is a prohibited state in nonextreme conditions. It therefore follows that the physical volume of a cell must lie in the range $V_{R} \leq V_{A}<V_{P}$. Because Eq. (58) implies that if $T=0$ then $V_{P}<V_{R}$, which cannot be true for any cell, Eq. (59) must be qualified with the proviso that it applies strictly to those cell volumes that are physically possible, expressed as

$$
V_{P \mid V_{R} \leq V_{A}<V_{P}}=V_{P \mid T=0}\left(1+\beta \frac{T}{\gamma H_{R}}\right) .
$$

In essence, $\beta$ represents a coefficient of thermal expansivity that applies universally to the constituent cells in any system, where $\beta=1 /((2 / \alpha)-1) \approx 1 / 273.072$.

\section{ELECTROMAGNETISM}

\section{A. Electric charge}

A charged particle is defined herein as any unitary cell or multi-cell particle whose net $|\eta|$ value is greater than zero. Given the process by which the $n_{R}$ value of a cell is set during a phase transition, the probability that $n_{R}$ happens to be set to an integer is vanishingly small. Therefore, with asymptotic probability one, any given cell in a physical system has an energy imbalance such that $|\eta|>0$.

The mechanism given by Eq. (38) for setting the $n_{G}$ value of a cell dictates that $\eta$ must describe a circular function. The cusp of $\eta$ is defined herein as the point on the function at which $|\eta|=0.5$, denoting the maximum energy imbalance that a cell can have. The cusp therefore defines the value of $\eta$ at which the electric charge carried by a cell is (exactly) maximal. As with the improbability of a zero value, the probability that the $|\eta|$ of a cell is set to a value of precisely 0.5 during a phase transition is vanishingly small. Therefore, with asymptotic probability one, the greatest $|\eta|$ value of a cell in a physical system is very nearly, but not precisely, 0.5 .

A cusp range is defined herein as $\eta= \pm\left(0.5-\delta_{\eta}\right)$ such that $0<\delta_{\eta} \ll 0.5$, and a cell whose $\eta$ value lies within the cusp range is defined as possessing a unit charge. Because electrons and protons carry unit charges of opposite sign, their respective (net) $\eta$ values must lie within the cusp range but on opposite sides of the cusp, where the cusp denotes an $\eta^{-}$value such that $\eta=-0.5$. Given that the energy imbalance of a cell is (very nearly) maximal when its $|\eta|$ is within the cusp range and decreases as $|\eta|$ approaches zero, it follows that the magnitude of electric charge must diminish as $|\eta|$ approaches zero. Therefore, a cell carries a fractional charge when $0<|\eta|<\left(0.5-\delta_{\eta}\right)$.

As an energy-abundant $\left(\eta^{-}\right)$single-cell particle, an electron carries a negative unit charge. Unlike an electron, a proton is an energy-deficient $\left(\eta^{+}\right)$rank-1 multi-cell cluster whose positive unit charge is due to a net $\eta^{+}$ difference between the total $\eta$ of its $\eta^{+}$cells and the total $\eta$ of its $\eta^{-}$cells. Based on Eq. (9), the average groundstate photino counts of electrons, protons and neutrons are $n_{\mathrm{e}} \approx 1.235589965 \times 10^{20}, n_{\mathrm{p}} \approx 2.268731818 \times 10^{23}$ and $n_{\mathrm{n}} \approx 2.271859081 \times 10^{23}$, respectively. Given that an electron is a unitary LDT cell and positing that the constituent cells inside a nucleon have an average $n_{G}$ value equal to $n_{\mathrm{e}}$, the total number of cells in an average neutron can be calculated as $\left\lfloor n_{\mathrm{n}} / n_{\mathrm{e}}\right\rfloor=1,838$ and the total number of cells in an average proton as $\left\lfloor n_{\mathrm{p}} / n_{\mathrm{e}}\right\rfloor=1,836$. Because an average neutron is observed to be electrically neutral, it can be deduced that the total $\eta$ of its $\eta^{+}$cells must offset the total $\eta$ of its $\eta^{-}$cells, which further suggests that an average neutron contains an equal number of $\eta^{+}$and $\eta^{-}$cells, namely $919 \eta^{+}$cells and $919 \eta^{-}$cells. Furthermore, because a proton carries a net positive charge and contains two cells fewer than an average neutron, it can be deduced that a proton is rendered electrically positive by a deficit of exactly two $\eta^{-}$cells, whereby an average proton consists of $919 \eta^{+}$cells and $917 \eta^{-}$cells. 
A cell is in its ideal volumetric state when it is energyneutral such that its $\eta=0$. Pursuant to Eq. (14), an energy imbalance (i.e., $|\eta|>0$ ) induces potential energy in a cell, which the cell must then reduce maximally in each time step in accordance with the autonomic principle. However, such a reduction cannot occur because it requires the cell to relinquish or acquire a fractional photino, which is a physical impossibility.

The $\eta$ value of a cell further implies an equivalent fractional photino mass value $m_{\eta}$, which, based on Eq. (5), is given by the relationship $m_{\eta}=\eta h v_{0} / c^{2}$. Thus, $m_{\eta}$ describes the deviation of the actual photino mass of the cell from its ideal (energy-neutral) photino mass. Because $\eta$ can be either positive or negative, so can $m_{\eta}$. A negative $m_{\eta}$ denotes an excess of mass that the cell seeks to relinquish in pursuit of energy neutrality, while a positive $m_{\eta}$ denotes a deficiency of mass that the cell must acquire to attain energy neutrality. However, because photinos are quantized, a cell can neither relinquish nor acquire a fractional photino mass, whereby $m_{\eta}$ remains an intrinsic feature of every cell under nonextreme conditions.

Let $\vec{c}_{m \mid \eta=0}$ be the radius vector of the center of mass of a cell in the theoretical case in which the cell is energy neutral such that $n_{R}=n_{G}$ and thus $\eta=m_{\eta}=0$. Given that, with asymptotic probability one, any given cell in a physical system has an energy imbalance, it follows that the center of mass of a cell is almost surely displaced from the center of mass that it would have had if its $\eta$ was zero. If $\vec{R}_{\eta}$ is the displacement of the center of mass of a cell from its energy-neutral point $c_{m \mid \eta=0}$ due to its extant $\eta$ (equivalently, $m_{\eta}$ ) value, then

$$
\vec{R}_{\eta} \triangleq \vec{c}_{m \mid \eta=0}-\vec{c}_{m \mid \eta}
$$

where $\vec{c}_{m \mid \eta}$ denotes the radius vector of the displaced center of mass

The polar chord of a cell can be regarded as an imaginary rod over which the entire photino content of the cell (and equivalently its mass) is distributed. The photino concentration at any given point $x$ on the polar chord is then described by the amplitude $\mathbb{A}_{x}$ of the stationary photino wave of the cell, where Eq. (35) gives the associated wave function $\psi(x)$. Given that $\psi(x)$ mirrors the distribution of neighboring cell densities, the distribution of photinos inside a cell, including the fractional photino $\eta h$ (and hence its mass $m_{\eta}$ ), reflects this geometry. Moreover, because it represents the fractional photino mass difference between the actual and ideal total mass values of a cell, $m_{\eta}$ is not associated with any specific photino located at a well-defined geometric coordinate within the cell. Rather, the location of $m_{\eta}$ within a cell can be described as a floating coordinate that lies always on the polar chord of the cell and shifts in response to changes in the density distribution of its neighboring cells. $\vec{R}_{\eta}$ is therefore coaxial with the polar chord of a cell, while any non-coaxial shift in the location of $m_{\eta}$ must, by definition, cause the axis of $\vec{R}_{\eta}$ (and hence the polar chord) of the cell to pivot correspondingly about its center of mass $c_{m \mid \eta}$

Furthermore, as the excess fractional photino mass of an energy-abundant cell, a negative-valued $m_{\eta}$ lies on the (higher-density) southern side of the point $c_{m \mid \eta=0}$ along the polar chord of the cell. Conversely, as the implied deficiency of a fractional photino mass, a positive-valued $m_{\eta}$ lies on the northern side of $c_{m \mid \eta=0}$ along the polar chord of a cell.

Let $\overrightarrow{\mathbb{d}}$ be a normalized measure of the displacement of the center of mass of a cell (in its ground state) due to $\eta$ such that

$$
\overrightarrow{\mathbb{d}} \triangleq \frac{\vec{R}_{\eta}}{\mathbb{r}}
$$

where $\mathbb{r} \triangleq\left(\lambda_{1} / 2 \pi\right)$, and $\lambda_{1}$ is the fundamental mode wavelength of the stationary photino wave of the cell. The quantity $\mathbb{r}$ thus describes a standard measure of length within any given cell, against which the extant $R_{\eta}$ value of the cell can be compared to give a normalized measure of the displacement of the center of mass of the cell due to $\eta$.

Based on Eq. (33), a normalized measure of the displacement of a cell (denoted by $\oslash$ ) from its equilibrium location within the ambient medium $\odot$ can be described by the quantity $\mathcal{D}_{\eta}$ such that

$$
\mathcal{D}_{\eta}=\left(\frac{\rho_{\oslash}-\bar{\rho}_{\odot}}{\rho_{\oslash}}\right)=\left(1-\frac{\bar{\rho}_{\odot}}{\rho_{\oslash}}\right) .
$$

(Note: $\oslash$ hereinafter denotes any cell or particle that is the principal object of the relationship under discussion.)

Let $U_{\eta}$ be the potential energy induced in a cell $\oslash$ by the displacement of its center of mass from the energyneutral point $c_{m \mid \eta=0}$. A normalized quantity $\mathcal{U}_{\eta}$ can be then defined as the stored potential energy $U_{\eta}$ per unit displacement of $\oslash$ from its equilibrium location within the ambient medium $\odot$ such that $\mathcal{U}_{\eta}=U_{\eta} / \mathcal{D}_{\eta}$. Based on Eq (14), $\mathcal{U}_{\eta}$ can be stated as a function of the ground-state energy content $H_{G} v_{0}$ of $\oslash$ and the normalized displacement of its center of mass by the relationship $U_{\eta}=\left(H_{G} v_{0} / 2\right)\left(\vec{R}_{\eta} / \mathrm{r}\right)^{2}$, expressed equivalently as

$$
U_{\eta}=\frac{1}{2} n_{G} h v_{0} \mathcal{D}_{\eta}(\overrightarrow{\mathbb{d}})^{2}
$$

This equation gives an internal measure of $U_{\eta}$, from the perspective of $\oslash$. 
Pursuant to Eq. (14), an external measure of the potential energy stored in $\oslash$ (i.e., from the perspective of its host system) is given by

$$
U_{\eta}=\frac{1}{2} k_{\eta}(\vec{r})^{2},
$$

where $k_{\eta}$ is a case-specific proportionality coefficient and $\vec{r}=\left(\vec{c}_{m \mid \eta}-\vec{c}_{0}\right)$, while $\vec{c}_{0}$ is the radius vector of the collective center of mass $c_{0}$ of all elements within an imaginary spherical surface $\mathcal{S}$ of radius $r$. By definition, therefore, the center of mass of $\oslash$ lies on $\mathcal{S}$ at a distance $r$ from $c_{0}$. Equating the right-hand sides of Eq. (64) and Eq. (65), the coefficient $k_{\eta}$ can be derived as

$$
k_{\eta}=\frac{n_{G} h v_{0}}{(\vec{r})^{2}} \mathcal{D}_{\eta}(\overrightarrow{\mathbb{d}})^{2} .
$$

If $\vartheta$ is the angle subtended by the axes of $\overrightarrow{\mathbb{d}}$ and $\vec{r}$, as measured relative to the positive direction of the axis of $\vec{r}$, then $0 \leq \pm \vartheta \leq \pi$ (see Fig. 8). The ensuing geometry implies that $(\overrightarrow{\mathbb{d}})^{2}=(\overrightarrow{\mathbb{d}} \cos \vartheta)^{2}+(\overrightarrow{\mathbb{d}} \sin \vartheta)^{2}$, whereby Eq. (64) becomes

$$
U_{\eta}=\frac{1}{2} n_{G} h v_{0} \mathcal{D}_{\eta}\left((\overrightarrow{\mathbb{d}} \cos \vartheta)^{2}+(\overrightarrow{\mathbb{d}} \sin \vartheta)^{2}\right) .
$$

Separating the two components of this relationship, $U_{\eta}^{\prime}=$ $n_{G} h v_{0} \mathcal{D}_{\eta}(\overrightarrow{\mathbb{d}} \cos \vartheta)^{2} / 2$ describes the potential energy associated with the component of $\overrightarrow{\mathbb{d}}$ that is coaxial with $\vec{r}$ and $U_{\eta}^{\prime \prime}=n_{G} h v_{0} \mathcal{D}_{\eta}(\overrightarrow{\mathbb{d}} \sin \vartheta)^{2} / 2$ describes the potential energy associated with the component of $\overrightarrow{\mathbb{d}}$ that is orthogonal to $\vec{r}$.

Similarly, from Eq. (66), the factors of $k_{\eta}$ associated with the coaxial and orthogonal components of $\overrightarrow{\mathbb{d}}$ can be separated as $k_{\eta}^{\prime}=\left(n_{\oslash} h v_{0} / r^{2}\right) \mathcal{D}_{\eta}(\overrightarrow{\mathbb{d}} \cos \vartheta)^{2}$ and $k_{\eta}^{\prime \prime}=$ $\left(n_{\oslash} h v_{0} / r^{2}\right) \mathcal{D}_{\eta}(\overrightarrow{\mathbb{d}} \sin \vartheta)^{2}$, respectively, where $n_{\oslash}$ is the ground-state photino count of cell $\oslash$. Based on Eq. (7), $n_{\odot} v_{0}=v_{\oslash}$, where $v_{\varnothing}$ is the fundamental harmonic frequency of the stationary photino wave of $\oslash$. Furthermore, $v_{\odot}$ can be expressed in terms of the wavelength of the stationary photino wave in its fundamental mode as $v_{\oslash}=c / \lambda_{1(\oslash)}$, whereby $n_{\oslash} h v_{0}=$ $h c / \lambda_{1(\oslash)}$.

Therefore, the coaxial and orthogonal factors of $k_{\eta}$ can be stated as $k_{\eta}^{\prime}=\left(h c / \lambda_{1(\odot)} r^{2}\right) \mathcal{D}_{\eta}(\overrightarrow{\mathbb{d}} \cos \vartheta)^{2}$ and $k_{\eta}^{\prime \prime}=\left(h c / \lambda_{1(\oslash)} r^{2}\right) \mathcal{D}_{\eta}(\overrightarrow{\mathbb{d}} \sin \vartheta)^{2}$, respectively. From Eq. (16), the potential energy $U_{\eta}$ produces a restoring force $\overrightarrow{\mathcal{F}}_{\eta}=-k_{\eta} \vec{R}_{\eta}$. It follows that $\vec{F}_{\eta}$, the coaxial component of $\overrightarrow{\mathcal{F}}_{\eta}$, is given as

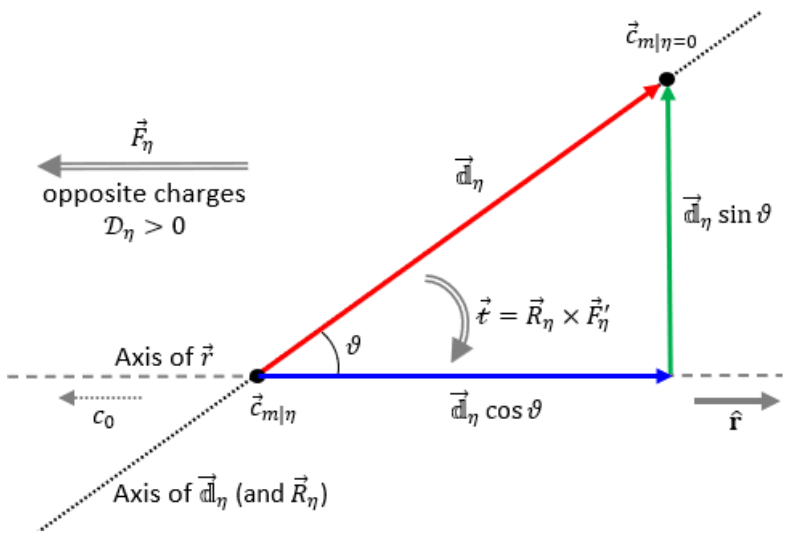

FIG. 8. Angle $\vartheta$ subtended by axes of $\overrightarrow{\mathbb{d}}_{\eta}$ and $\vec{r}$. Torque $\vec{t}$ acts to pivot the axis of $\vec{R}_{\eta}$, aligning it with the axis of $\vec{r}$, thus causing cell polarization. Electrostatic force $\vec{F}_{\eta}$ between opposite charges is attractive (i.e., acts in the direction antiparallel to $\hat{\mathbf{r}}$ ) if $\mathcal{D}_{\eta}>0$, whereas it is repulsive (i.e., acts in the direction of $\hat{\mathbf{r}}$ ) if $\mathcal{D}_{\eta}<0$. Conversely, $\vec{F}_{\eta}$ between like charges is repulsive if $\mathcal{D}_{\eta}>0$ and attractive if $\mathcal{D}_{\eta}<0$.

$$
\vec{F}_{\eta}=-\frac{h c}{\lambda_{1(\oslash)} r^{2}} \mathcal{D}_{\eta}(\overrightarrow{\mathbb{d}} \cos \vartheta)^{2} \vec{R}_{\eta} \cos \vartheta
$$

while $\vec{F}_{\eta}^{\prime}$, the orthogonal component of $\overrightarrow{\mathcal{F}}_{\eta}$, is given as

$$
\vec{F}_{\eta}^{\prime}=-\frac{h c}{\lambda_{1(\varnothing)} r^{2}} \mathcal{D}_{\eta}(\overrightarrow{\mathbb{d}} \sin \vartheta)^{2} \vec{R}_{\eta} \sin \vartheta
$$

\section{B. Polarization}

The autonomic principle dictates that $U_{\eta} \rightrightarrows 0$ and, according to Eq. (67), $\vartheta$ and $\mathcal{D}_{\eta}$ are the only arguments of $U_{\eta}$ whose values are variable under nonextreme conditions. Hence, $U_{\eta} \rightrightarrows 0$ implies that $\sin \vartheta \rightrightarrows 0$ and $\mathcal{D}_{\eta} \rightrightarrows 0$. If $r$ and $\mathcal{D}_{\eta}$ are held constant and $\sin \vartheta \neq 0$, then based on Eq. (69), it follows that $\vec{F}_{\eta}^{\prime} \rightrightarrows 0$ as $\sin \vartheta \rightrightarrows 0$, whereby there exists a torque $\vec{t}$ that impels $\vec{R}_{\eta}$ to rotate such that its axis pivots about the point $c_{m \mid \eta}$ towards the axis of $\vec{r}$, given by

$$
\vec{t}=\vec{R}_{\eta} \times \vec{F}_{\eta}^{\prime} .
$$

A cell is described as polarized if $\sin \vartheta \simeq 0$, namely when the axis of $\vec{R}_{\eta}$ (and hence the polar axis) of the cell is as closely aligned as possible with the axis of $\vec{r}$. The polarization of a charged particle therefore occurs because of the autonomic propensity of its polar axis to pivot into alignment with the axis connecting its center of mass to the collective center of mass of the local system enclosed in $\mathcal{S}$. As indicated earlier, the physical mechanism behind 
the rotation of the axis of $\vec{R}_{\eta}$ entails a (non-coaxial) shift in the location of $m_{\eta}$ within the cell.

\section{Universal electrostatic force function and Coulomb's law}

If a charged cell is polarized completely, we have $\vartheta=0$ or $\pi$, whereby $\cos \vartheta= \pm 1$ and hence $\vec{R}_{\eta} \cos \vartheta= \pm \vec{R}_{\eta}$. Replacing $\overrightarrow{\mathbb{d}}$ in Eq. (68) with the right-hand side of Eq. (62) and $\vec{R}_{\eta} \cos \vartheta$ with $\pm \vec{R}_{\eta}$, the force acting on a polarized cell $\oslash$ in either direction along the axis of $\vec{r}$ is given as $\vec{F}_{\eta}=-\left(h c / \lambda_{1(\oslash)} r^{2}\right) \mathcal{D}_{\eta}\left[\left( \pm \vec{R}_{\eta} / \mathrm{r}_{\oslash}\right)^{2}\left( \pm \vec{R}_{\eta}\right)\right]$. According to Eq. (46), $\lambda_{1(\oslash)}=\lambda_{C(\oslash)}$, whereby the previous equation becomes

$$
\vec{F}_{\eta}=-\frac{h c}{\lambda_{C(\oslash)} r^{2}} \mathcal{D}_{\eta}\left[\left(\frac{ \pm \vec{R}_{\eta}}{\mathrm{r}_{\oslash}}\right)^{2}\left( \pm \vec{R}_{\eta}\right)\right]
$$

By definition, the center of mass of a system is the point at which the sum of the moments of the masses distributed over the system, as a proportion of the total mass of the system, has a net zero value. Therefore, it can be deduced that the moment $\vec{M}_{\eta}$ produced by $m_{\eta}$ with respect to the (undisplaced) center of mass of a cell in its ground state is described by $\vec{M}_{\eta}=m_{\eta}\left(\vec{R}_{\eta}\right) / m_{G}$. Because $m_{\eta} / m_{G}=\eta / n_{G}$, the previous relationship can be expressed as

$$
\vec{M}_{\eta}=\vec{R}_{\eta}\left(\frac{\eta}{n_{G}}\right)
$$

In the context of photodynamics, the electric dipole moment $\vec{d}$ of a cell is defined by the relation $\vec{d} \propto \vec{M}_{\eta}$. Positing that the elementary charge $e \approx 1.602 \times 10^{-19} \mathrm{C}$ is a universal constant of proportionality associated with this relation, we obtain

$$
\vec{d}=e \vec{R}_{\eta}\left(\frac{\eta}{n_{G}}\right)
$$

Given the mechanism by which $\eta$ is set for a cell, the values of the arguments of this function cannot change under any nonextreme conditions. Therefore, an electric dipole moment is an intrinsic feature of every cell and, by extension, of every rank-1 multi-cell cluster, whereby its value remains permanently set under all but extreme conditions.

From Eq. (73), the $\vec{R}_{\eta}$ of a cell $\oslash$ (in its ground state) can be described in terms of its electric dipole moment as

$$
\vec{R}_{\eta}=\frac{n_{\oslash} \vec{d}}{e \eta}
$$

whereby Eq. (71) can also be written equivalently as $\vec{F}_{\eta}=$ $-\left(h c / \lambda_{C(\oslash)} r^{2}\right) \mathcal{D}_{\eta}\left[\left( \pm n_{\oslash} \vec{d} / e \eta \mathrm{r}_{\oslash}\right)^{2}\left( \pm \vec{R}_{\eta}\right)\right]$. The righthand side of this equation can then be multiplied by $(2 \pi \alpha) /(2 \pi \alpha)$ to give

$$
\vec{F}_{\eta}=-\frac{\alpha}{\alpha} \frac{\hbar c}{\pi_{C(\oslash)} r^{2}} \mathcal{D}_{\eta}\left[\left( \pm \frac{n_{\oslash} \vec{d}}{e \eta \mathbb{r}_{\oslash}}\right)^{2}\left( \pm \vec{R}_{\eta}\right)\right]
$$

where $\hbar=h / 2 \pi$ and $\lambda_{C(\varnothing)}=\lambda_{C(\oslash)} / 2 \pi$. Restating Eq. (74) as $n_{\oslash} \vec{d} / \eta=e \vec{R}_{\eta(\oslash)}$ and replacing the term $n_{\oslash} \vec{d} / \eta$ in Eq. (75) accordingly, we get

$$
\vec{F}_{\eta}=-\alpha \frac{\hbar c}{e^{2}}\left(\frac{e^{2}}{r^{2}}\right) \mathcal{D}_{\eta}\left[\left(\frac{ \pm \vec{R}_{\eta(\oslash)}}{\alpha \lambda_{C(\oslash)}}\right)\left(\frac{ \pm \vec{R}_{\eta(\oslash)}}{\mathbb{r}_{\oslash}}\right)^{2}\right]
$$

By evaluating the constants, it can be readily shown that $\alpha\left(\hbar c / e^{2}\right)=\mathbb{k}_{e} \approx 8.988 \times 10^{9} \mathrm{~N} \mathrm{~m}^{2} \mathrm{C}^{-2}$, where $\mathbb{k}_{e}$ is Coulomb's constant. In addition, by defining a constant $\epsilon_{0} \approx 8.854 \times 10^{-12} \mathrm{C}^{2} \mathrm{~N}^{-1} \mathrm{~m}^{-2}$ as the permittivity of free space, Coulomb's constant can be stated alternatively as $\mathbb{k}_{e}=1 / 4 \pi \epsilon_{0}$.

Replacing $\alpha\left(\hbar c / e^{2}\right)$ with $\mathbb{k}_{e}$, Eq. (76) becomes $\vec{F}_{\eta}=$ $-\mathbb{k}_{e}\left(e^{2} / r^{2}\right) \mathcal{D}_{\eta}\left[\left( \pm \vec{R}_{\eta(\oslash)} / \alpha \lambda_{C(\oslash)}\right)\left( \pm \vec{R}_{\eta(\oslash)} / \mathbb{r}_{\oslash}\right)^{2}\right]$. If a quantity $q_{0}$ is defined such that $q_{0}=e$, then the previous equation can be expressed as

$$
\vec{F}_{\eta}=-\mathbb{k}_{e} \frac{q_{0} q_{0}}{r^{2}} \mathcal{D}_{\eta}\left[\left(\frac{ \pm \vec{R}_{\eta(\oslash)}}{\alpha \lambda_{C(\oslash)}}\right)\left(\frac{ \pm \vec{R}_{\eta(\oslash)}}{\mathbb{r}_{\oslash}}\right)^{2}\right] .
$$

Let $\overrightarrow{\mathbb{d}}_{0} \triangleq\left(\vec{R}_{\eta(\oslash)} / \alpha \pi_{C(\oslash)}\right)$, while according to Eq. (62) $\left(\overrightarrow{\mathbb{d}}_{\oslash}\right)^{2}=\left(\vec{R}_{\eta(\oslash)} / \mathrm{r}_{\oslash}\right)^{2}$. Substituting the respective terms accordingly, Eq. (77) can be restated as

$$
\vec{F}_{\eta}=-\mathbb{k}_{e} \frac{q_{0} q_{0}}{r^{2}} \mathcal{D}_{\eta}\left[\left( \pm \overrightarrow{\mathbb{d}}_{0}\right)\left( \pm \overrightarrow{\mathbb{d}}_{\oslash}\right)^{2}\right]
$$

By definition, if $R_{\eta(\oslash)}=\alpha \pi_{C(\oslash)}$ then $\pm \overrightarrow{\mathbb{d}}_{0}=( \pm 1) \hat{\mathbf{r}}$, where $\hat{\mathbf{r}}$ is a unit vector outwardly normal to $\mathcal{S}$. Suppose that $\overrightarrow{\mathbb{d}}_{0}$ is associated with an ideal polarized cell, e, of electron mass that (theoretically) carries precisely one unit of negative charge; in other words, the $\eta$ value of the hypothetical cell $e$ is defined precisely by the identity $\eta_{\Theta}=-0.5$. In that case, $\overrightarrow{\mathbb{d}}_{0}=\overrightarrow{\mathbb{d}}_{\mathbb{e}}$ and $\alpha \lambda_{C(\oslash)}=\alpha \lambda_{C(\Theta)}$. Furthermore, by evaluating these constants we get $\alpha \pi_{C(\mathbb{e})}=r_{\Theta} \approx 2.818 \times 10^{-15} \mathrm{~m}$, where, by convention, 
$r_{\mathbb{e}}$ is described as the classical electron radius. Hence, given that the value of $R_{\eta(\Theta)}$ must be maximal because $\left|\eta_{\mathbb{e}}\right|=0.5$ defines the maximum (cusp) value of $\eta$, it follows that from the viewpoint of photodynamics, the classical electron radius represents the maximum possible displacement of the center of mass of an electron due to its energy imbalance $\eta$, stated as

$$
R_{\eta(\Theta)}=\alpha \pi_{C(\Theta)}=r_{\Theta}
$$

whereby $\overrightarrow{\mathbb{d}}_{\mathbb{e}}$ (and hence $\overrightarrow{\mathbb{d}}_{0}$ ) defines the unit vector $\hat{\mathbf{r}}$ through the relationship

$$
\frac{-\vec{R}_{\eta(\Theta)}}{r_{\Theta}}=-\overrightarrow{\mathbb{d}}_{\Theta}=(-1) \hat{\mathbf{r}} .
$$

Note that \pm is replaced by the explicit minus sign in the term $-\vec{R}_{\eta(\Theta)}$ in this equation because $巴$ is a polarized energy-abundant cell with $\eta_{\Theta}=-0.5$. Hence, the location of $m_{\eta}$ relative to $\vec{c}_{m \mid \eta=0}$ on the common axis of $\vec{R}_{\eta}$ and $\vec{r}$ must be on the side facing the center of mass $c_{0}$ of the system enclosed in $\mathcal{S}$, connoting $\vartheta=\pi$ and $\cos \vartheta=-1$, whereby the orientation of $\vec{R}_{\eta(\Theta)}$ must be antiparallel to the positive direction of $\vec{r}$, and therefore also of $\hat{\mathbf{r}}$.

The term $\left( \pm \overrightarrow{\mathbb{d}}_{\varnothing}\right)^{2}$ in Eq. (78) can be separated into its two factors such that each factor is associated with a specific charged particle. Based on Eq. (62), let $\pm \overrightarrow{\mathbb{d}}_{1} \triangleq$ $\left( \pm \vec{R}_{\eta(1)} / \mathbb{r}_{1}\right)$ and $\pm \overrightarrow{\mathbb{d}}_{2} \triangleq\left( \pm \vec{R}_{\eta(2)} / \mathbb{r}_{2}\right)$, where $\pm \overrightarrow{\mathbb{d}}_{1}$ is associated with the charged particle located at the center of mass of the system enclosed in $\mathcal{S}$, which can be called its primary charge; while $\pm \overrightarrow{\mathbb{d}}_{2}$ is associated with the charged particle centered on the surface $\mathcal{S}$, which can be called the test charge. Given further that the vectors $\overrightarrow{\mathbb{d}}_{1}$ and $\overrightarrow{\mathbb{d}}_{2}$ share a common axis, it follows that $\overrightarrow{\mathbb{d}}_{1} \cdot \overrightarrow{\mathbb{d}}_{2}=$ $\mathbb{d}_{1} \mathbb{d}_{2}$. Replacing the term $\left( \pm \overrightarrow{\mathbb{d}}_{\oslash}\right)^{2}$ accordingly with $\left( \pm \mathbb{d}_{1}\right)\left( \pm \mathbb{d}_{2}\right)$, and the term $\pm \overrightarrow{\mathbb{d}}_{0}$ with $-\hat{\mathbf{r}}$, Eq. (78) can be written equivalently as

$$
\vec{F}_{\eta}=\mathbb{k}_{e}\left(\frac{\left( \pm \mathbb{d}_{1} q_{0}\right)\left( \pm \mathbb{d}_{2} q_{0}\right)}{r^{2}}\right) \mathcal{D}_{\eta} \hat{\mathbf{r}}
$$

This equation depicts the classical view of the electrostatic force between a (single-particle) primary charge $\pm \mathbb{d}_{1} q_{0}$ and a (single-particle) test charge $\pm \mathbb{d}_{2} q_{0}$, where the medium $\odot$ surrounding both charges is treated as an empty vacuum with mass $m_{\odot}=0$, implying that $\bar{\rho}_{\odot}=0$ and hence $\mathcal{D}_{\eta}=\left(1-\bar{\rho}_{\odot} / \rho_{\odot}\right)=1$.

By contrast, according to photodynamics, the space surrounding each charge consists of contiguous physical elements, where an element may be a unitary cell or a multi-cell particle. Moreover, given that each element of the system enclosed in $\mathcal{S}$ almost surely possesses a (net) $|\eta|>0$, it follows that the complete system enclosed in $\mathcal{S}$ possesses a total charge $Q$ such that

$$
Q=q_{0} \sum_{i=1}^{N_{1}} \pm \mathbb{d}_{i}
$$

where $N_{1}$ is the total number of elements $i$ in the system enclosed in $\mathcal{S}$. Furthermore, a test charge may be a single charged particle or a multi-particle charged body composed of elements that may individually be unitary cells or multi-cell particles. If $N_{2}$ is the number of particles $j$ in the test charge, then its total charge $q$ is given by

$$
q=q_{0} \sum_{j=1}^{N_{2}} \pm \mathbb{d}_{j}
$$

Because the complete system enclosed in $\mathcal{S}$ consists not only of the primary charge but of all its elements collectively, $\left( \pm \mathbb{d}_{1} q_{0}\right)$ must be replaced with $Q$ in Eq. (81) to account for the total charge inside $\mathcal{S}$. In addition, because a test charge may be a unitary particle or a multiparticle cluster, $\left( \pm \mathbb{d}_{2} q_{0}\right)$ must be replaced with $q$, whereby Eq. (81) reduces to the universal electrostatic force function $\vec{F}_{\eta}=\mathbb{k}_{e}\left(Q q / r^{2}\right) \mathcal{D}_{\eta} \hat{\mathbf{r}}$, which can be written in expanded form as

$$
\vec{F}_{\eta}=\mathbb{k}_{e} \frac{Q q}{r^{2}}\left(1-\frac{\bar{\rho}_{\odot}}{\rho_{q}}\right) \hat{\mathbf{r}},
$$

where $\rho_{q}$ is the photino density of the test charge $q$, and $\bar{\rho}_{\odot}$ is the average density of the medium surrounding $q$. If space is treated as an empty vacuum (i.e., $\mathcal{D}_{\eta}=1$ ), then Eq. (84) is identical in its form to the classical formula for Coulomb's law of electrostatic force, given as

$$
\overrightarrow{\mathbb{F}}_{\mathrm{C}}=\mathbb{k}_{e} \frac{Q q}{r^{2}} \hat{\mathbf{r}}
$$

where $\vec{F}_{\mathrm{C}}$ denotes the Coulomb force and $Q$ now denotes the (fixed) magnitude of the primary charge located a distance $r$ from the test charge $q$. However, notwithstanding this similarity in form, the universal electrostatic force function differs profoundly in its character from Coulomb's law in several respects.

Firstly, the quantity $\mathcal{D}_{\eta}=\left(1-\bar{\rho}_{\odot} / \rho_{q}\right)$ features prominently in Eq. (84) but does not appear in Coulomb's law. Based on the core hypothesis of photodynamics, physical space everywhere consists of contiguous cells with (positive) non-zero photino density values. Consequently, no region of space can have an average 
density of zero photinos per unit volume, rendering $\mathcal{D}_{\eta}=$ 1 a prohibited state for a physical system.

Moreover, if $\bar{\rho}_{\odot}<\rho_{q}$ then Eq. (63) implies $\mathcal{D}_{\eta}>0$. From Eq. (84), when $\mathcal{D}_{\eta}>0$ then the force $\vec{F}_{\eta}$ points in the $+\hat{\mathbf{r}}$ direction (i.e., $\vec{F}_{\eta}$ is repulsive) if the charges $Q$ and $q$ both have the same sign, whereas $\vec{F}_{\eta}$ points in the $-\hat{\mathbf{r}}$ direction (i.e., $\vec{F}_{\eta}$ is attractive) if $Q$ and $q$ have opposite signs. Thus, the condition $\bar{\rho}_{\odot}<\rho_{q}$ implies that $\vec{F}_{\eta}$ is repulsive between like charges and attractive between unlike charges, in agreement with Coulomb's law.

However, if $\bar{\rho}_{\odot}>\rho_{q}$ then Eq. (63) implies $\mathcal{D}_{\eta}<0$. From Eq. (84), when $\mathcal{D}_{\eta}<0$ then the force $\vec{F}_{\eta}$ points in the $-\hat{\mathbf{r}}$ direction (i.e., $\vec{F}_{\eta}$ is attractive) if the charges $Q$ and $q$ both have the same sign, whereas $\vec{F}_{\eta}$ points in the $+\hat{\mathbf{r}}$ direction (i.e., $\vec{F}_{\eta}$ is repulsive) if $Q$ and $q$ have opposite signs. The condition $\bar{\rho}_{\odot}>\rho_{q}$ thus implies an inversion of the direction conventionally associated with the electrostatic force between two nearby charges, whereby if (and only if) $\bar{\rho}_{\odot}>\rho_{q}$, then $\vec{F}_{\eta}$ is attractive between like charges and repulsive between unlike charges.

The density of a tangible body of matter is ordinarily higher than that of the medium that surrounds it, whereby the condition $\bar{\rho}_{\odot}<\rho_{q}$ holds true in all but exceptional cases. Consequently, the direction of $\vec{F}_{\eta}$ is normally observed to be repulsive between like charges and attractive between unlike charges. However, the exceptions to this norm, namely where the density of a tangible particle is lower than the density of its ambient medium, are of fundamental importance in determining the behavior and structure of matter at the subatomic scale. A notable exception where $\bar{\rho}_{\odot}>\rho_{q}$ is the case of the atomic nucleus in which $\mathcal{D}_{\eta}$ acts to invert the direction of $\vec{F}_{\eta}$, thus binding positively charged protons along with neutrons to form a nuclear cluster (see Sec. VII A).

Equation (84) also differs fundamentally from Coulomb's law in respect to its construction. Whereas the former is derived logically from first principles defined by the theoretical foundations of photodynamics, the latter is an empirical law constructed to best fit the data obtained from experimental observations.

Coulomb's law describes the electrostatic force between charged bodies of ordinary matter. Because ordinary matter is composed of tangible elementary particles that carry either no measurable charge (neutrons) or a unit charge (protons and electrons), the classical law is silent regarding the electrostatic force that may exist between particles that are either intangible or carry fractional charges. By contrast, Eq. (84) describes the general case of the electrostatic force between charged bodies composed of not only tangible particles of ordinary matter with net zero or unit charges, but also intangible cells in an LDI medium and calls that carry fractional charges with $|\eta|$ values that may lie anywhere in the range $0<|\eta|<\left(0.5-\delta_{\eta}\right)$.

According to Coulomb's law, the quantity $Q$ describes the magnitude of the primary charge in a system, while the space between the primary charge and the surface $\mathcal{S}$ is treated as an empty void. By contrast, Eq. (84) asserts that the value of $Q$ is fixed not by the magnitude of the primary charge alone but rather by the total charge present inside $\mathcal{S}$, which includes the primary charge as well as the sum of the charges carried by all of the other elements of the medium inside $\mathcal{S}$. Therefore, whereas Coulomb's law implies that the value of $Q$ is fixed independently of the distance $r$, according to Eq. (84) the value of $Q$ itself varies with the distance $r$ separating the primary and test charges.

Coulomb's law can therefore be described as a limiting case of the universal electrostatic force function given by Eq. (84) in which only charged bodies composed of ordinary matter are considered and space is assumed to be devoid of any material content.

\section{Spatial charge distribution}

Suppose that the medium of space that surrounds a primary charge inside $\mathcal{S}$ consists entirely of unitary cells that are in their equilibrium locations within the system such that $\rho_{i}-\bar{\rho}_{\odot} \simeq 0$ for every cell $i$ in the medium. In that case, the cells in the medium must be arranged in roughly concentric Gaussian order of radially diminishing density around the primary charge, whereby according to Eq. (29), there exists a density gradient $\vec{\nabla} \rho$ at any given point in the medium within $\mathcal{S}$. In addition, as described in Sec. VI B, autonomic compulsions dictate that these cells must tend towards a polarized state.

Equation (67) can be restated to describe the potential energy due to $\eta$ of any polarized cell present within the medium inside $\mathcal{S}$ as $U_{\eta}=\left(n_{G} h v_{0} \mathcal{D}_{\eta} / 2\right)( \pm \overrightarrow{\mathbb{d}})^{2}$. Substituting $\overrightarrow{\mathbb{d}}$ in this equation with the right-hand side of Eq. (62) yields $U_{\eta}=\left(n_{G} h v_{0} \mathcal{D}_{\eta} / 2\right)\left( \pm \vec{R}_{\eta} / \mathbb{r}\right)^{2}$. Given that $\mathbb{r}=\lambda_{1} / 2 \pi$ while $\lambda_{1}=\lambda_{C}$, it follows that $\mathbb{r}=\lambda_{C}$. Moreover, because the square of the vector $\pm \vec{R}_{\eta}$ connotes the square of its scalar value $\pm R_{\eta}$, the $U_{\eta}$ of a polarized cell inside $\mathcal{S}$ can be expressed as

$$
U_{\eta}=\frac{1}{2} n_{G} h v_{0} \mathcal{D}_{\eta}\left(\frac{ \pm R_{\eta}}{\lambda_{C}}\right)^{2}
$$

From Eq. (7), $n_{G} v_{0}$ connotes the fundamental harmonic frequency of the stationary photino wave of a cell as $n_{G} v_{0}=v_{1}=c / \lambda_{1}$, whereby Eq. (86) can be 
restated as $\quad U_{\eta}=\left(h c / 2 \lambda_{1}\right)\left(4 \pi^{2} \mathcal{D}_{\eta} / \lambda_{C}^{2}\right)\left( \pm R_{\eta}\right)^{2}$. Replacing $\lambda_{1}$ with $\lambda_{C}$ in this equation yields

$$
U_{\eta}=\frac{2 \pi^{2} h c}{\lambda_{C}^{3}} \mathcal{D}_{\eta}\left( \pm R_{\eta}\right)^{2}
$$

Because in the present context, $\rho_{i}-\bar{\rho}_{\odot} \simeq 0$ for every cell $i$ in the medium that surrounds the primary charge inside $\mathcal{S}$, it follows that $\mathcal{D}_{\eta} \simeq 0$ for each cell $i$ in the medium inside $\mathcal{S}$. By the autonomic principle, $U_{\eta} \rightrightarrows 0$. However, given that $\mathcal{D}_{\eta} \simeq 0$, while no other argument of the function described by Eq. (87) can change in value under nonextreme conditions, it follows that $U_{\eta} \simeq 0$, whereby the $U_{\eta}$ of every cell in the medium inside $\mathcal{S}$ is irreducible. If a quantity $k_{U} \triangleq \sqrt{\left(2 \pi^{2} h c \mathcal{D}_{\eta}\right) /\left(\lambda_{C}^{3}\right)}$ is defined as a cell-specific constant, then Eq. (87) becomes $U_{\eta}=k_{U}^{2}\left( \pm R_{\eta}\right)^{2}$, which can be stated alternatively as $\sqrt{U_{\eta}}=k_{U}\left( \pm R_{\eta}\right)$. If the medium inside $\mathcal{S}$ consists of $N_{\mathrm{m}}$ cells, then we have

$$
\sum_{i=1}^{N_{\mathfrak{m}}} \sqrt{U_{\eta, i}}=\sum_{i=1}^{N_{\mathfrak{m}}} k_{U, i}\left( \pm R_{\eta, i}\right),
$$

while the autonomic principle enforces the compulsion $\sum_{i=1}^{N_{\mathfrak{m}}} U_{\eta, i} \rightrightarrows 0$, whereby $\sum_{i=1}^{N_{\mathfrak{m}}} \sqrt{U_{\eta, i}} \rightrightarrows 0$. Now, although $k_{U, i}$ cannot be reduced further for any given cell $i$, the value of $\sum_{i=1}^{N_{\mathfrak{m}}} \sqrt{U_{\eta, i}}$ can decrease nevertheless if the quantity $\sum_{i=1}^{N_{\mathfrak{m}}}\left( \pm R_{\eta, i}\right)$ can be reduced further. It follows that if $\sum_{i=1}^{N_{\mathfrak{m}}}\left( \pm R_{\eta, i}\right) \nsim 0$, then

$$
\sum_{i=1}^{N_{\mathfrak{m}}}\left( \pm R_{\eta, i}\right) \rightrightarrows 0
$$

Given that the magnitude of the $R_{\eta}$ of a cell reflects the magnitude of its $\eta$ value, the condition of optimality $\sum_{i=1}^{N_{\mathfrak{m}}}\left( \pm R_{\eta, i}\right) \simeq 0$ is satisfied if and only if all cells in the medium within $\mathcal{S}$ are arranged such that the total $\eta$ of every pair of neighboring cells is minimal; in other words, if $j$ and $k$ are two neighboring cells in the medium, then $\forall j, k: \eta_{j}+\eta_{k} \simeq 0$. This arrangement implies that when the system is in equilibrium, each cell has an $\eta$ value such that the $\eta$ of each of its neighbors is as close as possible to its own $\eta$ in absolute value but with opposite sign. For example, if $\eta_{j}=-0.3$, then $\left(R_{\eta, j}+R_{\eta, k}\right) \simeq 0$ if and only if $\eta_{k} \simeq+0.3$.

Consequently, as the cells in the medium cluster in order of radially decreasing nearest density around a highdensity core, an additional substructure is imposed by the compulsion described by Eq. (89) on the spatial charge distribution in the system enclosed by $\mathcal{S}$. Within the characteristic density structure of the system, there exists a substructure such that the sign of $R_{\eta}$ alternates between negative and positive from cell to neighboring cell both radially and along the two (areal) dimensions on the surface $\mathcal{S}$, while the magnitude $\left|R_{\eta}\right|$ progressively decreases radially from the core. A characteristic Gaussian structure thus emerges in the spatial distribution of electric charge inside $\mathcal{S}$ as the system reaches equilibrium, while pairs of neighboring cells at each point in the medium inside $\mathcal{S}$ are rendered very nearly electrically neutral.

Given that the $\eta$ value of a cell can be anywhere in the range $-0.5 \leq \eta<+0.5$, it follows that the medium surrounding the primary charge inside $\mathcal{S}$ must consist predominantly of cells that individually carry fractional charges. In addition, quantitative individuality applies because $\eta \in \mathbb{R}$, whereby with asymptotic probability one $\left|\eta_{j}\right| \neq\left|\eta_{k}\right|$. Therefore, the $|\eta|$ of neighboring cells located on the surface $\mathcal{S}$ must differ in value, at least infinitesimally, whereby the magnitude of charge over the surface $\mathcal{S}$ is almost surely not identically uniform in the directions of increasing polar and azimuthal angles.

\section{E. Electric field, potential and current}

Within this emergent framework of Gaussian symmetry in the spatial distribution of electric charge, a scalenormalized measure of the electrostatic force at a point located a radial distance $r$ from a primary charge can be defined as the electric field $\overrightarrow{\mathbb{E}}$ at that point, which, based on Eq. (84), is given by

$$
\overrightarrow{\mathbb{E}}(r)=\frac{\vec{F}_{\eta}(r)}{q}=\mathbb{k}_{e} \frac{Q}{r^{2}} \mathcal{D}_{\eta} \hat{\mathbf{r}} .
$$

If space is treated as an empty vacuum such that $\mathcal{D}_{\eta}=1$, then Eq. (90) represents an alternative expression of Gauss's law for electric fields, given in differential form as $\vec{\nabla} \cdot \overrightarrow{\mathbb{E}}=\varrho / \varepsilon_{0}$, where $\varrho$ is the electric charge density.

A scale-normalized measure of the (electrostatic) potential energy $U_{\eta}$ can be defined similarly as the electric potential $\mathbb{V}$ at a point located on the surface $\mathcal{S}$ a radial distance $r$ from the primary charge such that

$$
\mathbb{V}(r)=\frac{U_{\eta}(r)}{q}
$$

According to the logic of the present model, electric current represents the cell-to-cell transmission of photinos in a conducting medium, where the photino transmission occurs through the entropic process of pass-through conduction as described in Sec. IV C. Hence, as a mechanism for transporting energy from point to point 
through the conduction of photinos, electric current is indistinguishable from the transmission of light (in the absence of resonance). This model further admits the possibility that free electrons play a dominant role in this process by acting as mobile carriers shunting unbound photinos from point to point across (low-conductivity LDI) spaces separating the molecules in a conducting material. If this is so, then the kinetic energy of such a carrier electron must come from the conversion of the potential energy induced in it by the absorption of photinos received from the (bound) outer electrons in the molecules with which the free electron comes into contact.

Furthermore, from Eq. (91) it follows that the autonomic compulsion $U_{\eta} \rightrightarrows 0$ implies $\mathbb{V} \rightrightarrows 0$, whereby the direction of photino conduction is regulated by $\mathbb{V}$ such that the photinos flow always from cells with higher electric potential to neighboring cells with lower electric potential. In addition, using the standard methodology of classical electrostatics for formulating key relationships associated with electric potential, it can be easily deduced that the potential difference between any two points $A$ and $B$ in an electric field produced by a charge $Q$ is described by $\mathbb{V}_{\mathrm{B}}-\mathbb{V}_{\mathrm{A}}=\mathbb{k}_{e} Q\left[1 / r_{\mathrm{B}}-1 / r_{\mathrm{A}}\right] \mathcal{D}_{\eta}$, where $r_{\mathrm{A}}$ and $r_{\mathrm{B}}$ are the distances of $A$ and $B$, respectively, from the center of mass of $Q$. If $\mathrm{A}$ is taken to be sufficiently distant from the center of mass of $Q$ such that $1 / r_{\mathrm{A}} \rightarrow 0$, then it follows that the electric potential due to $Q$ at any distance $r$ from its center of mass is given by

$$
\mathbb{V}=\mathbb{k}_{e} \frac{Q}{r} \mathcal{D}_{\eta}
$$

From Eq. (91), we have $U_{\eta}=q \mathbb{V}$, and dividing the magnitude of the electrostatic force $F_{\eta}$ as per Eq. (84) by Eq. (92) yields $F_{\eta} / \mathbb{V}=q / r$, whereby

$$
U_{\eta}=q \mathbb{V}=F_{\eta} r
$$

\section{F. Magnetic field}

A magnetic field $\vec{B}$ is conventionally described in terms of the magnetic force $\vec{F}_{B}$ experienced by a charged particle $q$ in relative motion with respect to $\vec{B}$, as expressed by the Lorentz equation for magnetic force $\vec{F}_{B}=q(\vec{v} \times \vec{B})$, where $\vec{v}$ is the velocity of the charged particle with respect to $\vec{B}$. The magnitude of the magnetic field is therefore given by the relationship $|\vec{B}|=\left|\vec{F}_{B}\right| / q|\vec{v}| \sin \theta$, where $\theta$ is the angle between $\vec{v}$ and $\vec{B}$.

According to the present model, while magnetic force is experienced by a charged particle if and only if it is in relative motion with respect to an ambient magnetic field as described by the Lorentz equation, contrary to the conventional view, the source of a magnetic field is not the motion of the charged particle. Rather, photodynamics describes a magnetic field as an inherent feature of space that arises ultimately from the electric charges and photino densities of the individual cells that collectively constitute any given region of space, as follows.

Replacing $U_{\eta}$ and $F_{\eta}$ in Eq. (93) by the right-hand sides of Eq. (64) and Eq. (84), respectively, yields

$$
\frac{1}{2} n_{q} h v_{0} \mathcal{D}_{\eta}\left(\mathbb{d}_{q}\right)^{2}=\left(\frac{1}{4 \pi \epsilon_{0}} \frac{Q q}{r^{2}} \mathcal{D}_{\eta}\right) r
$$

where $n_{q}=\sum_{i=1}^{N} n_{G, i}$ and $\mathbb{d}_{q}=(1 / N) \sum_{i=1}^{N}\left(R_{\eta, i} / \mathrm{r}_{\mathrm{i}}\right)$ if $q$ is composed of $N$ particles.

Given that $q$ consists of ordinary matter, its elemental composition must be a combination of electrons, protons and neutrons. Now, because $\eta_{\mathrm{e}}$ and $\eta_{\mathrm{p}}$ both lie in the cusp range, it follows that $R_{\eta(\mathrm{e})}$ and $R_{\eta(\mathrm{p})}$ must be (very nearly) maximal in absolute value. Hence, based on Eq. (79) it can be deduced that $R_{\eta(\mathrm{e})} \approx \alpha \lambda_{C(\mathrm{e})}$ and $R_{\eta(\mathrm{p})} \approx \alpha \lambda_{C(\mathrm{p})}$, while $R_{\eta(\mathrm{n})} \approx 0$ by definition because $\eta_{\mathrm{n}} \approx 0$, implying that $R_{\eta(\mathrm{e})} \approx \alpha \mathrm{r}_{\mathrm{e}}$ and $R_{\eta(\mathrm{p})} \approx \alpha \mathrm{r}_{\mathrm{p}}$, whereby $\mathbb{d}_{q} \approx \alpha$.

Substituting accordingly for $\mathbb{d}_{q}$ and multiplying both sides by $2 / \mathcal{D}_{\eta}$, Eq. (94) becomes $n_{q} h v_{0} \alpha^{2} \approx Q q / 2 \pi \epsilon_{0} r$, which can be restated as $n_{q} h / q \approx Q / \alpha^{2} \epsilon_{0}\left(2 \pi r v_{0}\right)$. If angular speed $\omega_{r}$ is defined such that $\omega_{r}=2 \pi v_{r}$, where $v_{r}=r v_{0}$ is the linear speed, then the previous equation can be expressed equivalently as $n_{q} h / q \approx Q / \alpha^{2} \epsilon_{0} \omega_{r}$. The left-hand side of this equation is then a general description of photino content per unit charge, which, from the perspective of photodynamics, defines the magnetic flux $\Phi_{B}$ associated with a cell located at any given point in space. Magnetic flux is thus described herein by the relationship

$$
\Phi_{B}=\frac{n_{q} h}{q} \approx \frac{Q}{\alpha^{2} \epsilon_{0} \omega_{r}} .
$$

Note that the term $Q / \alpha^{2} \epsilon_{0} \omega_{r}$ in this equation can be described as an allegorical representation of magnetic flux in which an imaginary point charge can be said to be orbiting an origin with a radius $r$ at an angular speed $\omega_{r}$, while $Q$ is the charge enclosed inside the surface $\mathcal{S}$. This representation essentially depicts the classical view in which imaginary revolving point charges, connoting virtual current loops, are regarded as the ultimate sources of magnetic effects.

In agreement with classical magnetostatics, the unit density of magnetic flux over any given surface of area $A$ defines the magnitude of the magnetic field, such that $|\vec{B}|=\Phi_{B} /|\vec{A}| \cos \theta$, where $\vec{A}$ is a vector of magnitude $A$ normal to the surface, and $\theta$ is the angle between $\vec{B}$ and $\vec{A}$. This equation can then be written alternatively as 


$$
\Phi_{B}=|\vec{B}||\vec{A}| \cos \theta=\vec{B} \cdot \vec{A} .
$$

Notably, the relationship $\Phi_{B}=n_{q} h / q$ in Eq. (95) gives an unambiguous description of the ontology of magnetic flux as the photino content per unit charge at any given location in space - and, by extension, describes the precise physical character of magnetic fields.

\section{G. Permanent electric dipole moment}

Given that $\left|\eta_{\mathrm{e}}\right|$ lies in the cusp range, $R_{\eta(\mathrm{e})}$ must be very nearly maximal in value, whereby from Eq. (79) we have $R_{\eta(\mathrm{e})} \approx \alpha \pi_{C(\mathrm{e})}$. Replacing $R_{\eta(\mathrm{e})}$ in this equation with the right-hand side of Eq. (74) for the case of a physical electron gives $\left(n_{\mathrm{e}} \vec{d}_{\mathrm{e}} / e \eta_{\mathrm{e}}\right) \approx \alpha \lambda_{C(\mathrm{e})} \hat{\mathrm{r}}$. Rearranging the terms in this equation, the electric dipole moment of an electron is given by

$$
\vec{d}_{\mathrm{e}} \approx \eta_{\mathrm{e}}\left(\frac{e \alpha \pi_{C(\mathrm{e})}}{n_{\mathrm{e}}}\right) \hat{\mathbf{r}}
$$

Because $\eta_{\mathrm{e}}=-\left(0.5-\delta_{\eta}\right)=-\left(1-2 \delta_{\eta}\right) / 2$, Eq. (97) becomes $\vec{d}_{\mathrm{e}} \approx-\left(1-2 \delta_{\eta}\right)\left(e \alpha \mathrm{r}_{\mathrm{e}} / 2 n_{\mathrm{e}}\right) \hat{\mathbf{r}}$, which can be restated as

$$
\vec{d}_{\mathrm{e}} \approx-\frac{e\left(\alpha \pi_{C(\mathrm{e})}\right)\left(1-2 \delta_{\eta}\right)}{2 n_{\mathrm{e}}} \hat{\mathbf{r}} .
$$

Neglecting the cusp range limit $\delta_{\eta} \ll 0.5$ and multiplying the right-hand side of Eq. (98) by $\left(h v_{0} / c^{2}\right) /\left(h v_{0} / c^{2}\right)$, the approximate value of the permanent electric dipole moment of an electron can be determined as

$$
\vec{d}_{\mathrm{e}} \approx-\frac{e m_{h}\left(\alpha \pi_{C(\mathrm{e})}\right)}{2 m_{\mathrm{e}}} \hat{\mathbf{r}} \sim-1.827 \times 10^{-54} \mathrm{C} \mathrm{m} .
$$

Replacing $\alpha \lambda_{C(\mathrm{e})}$ in this equation with $\alpha \pi_{C(\mathrm{p})}$ for the case of a proton and given that $\eta_{\mathrm{p}}=+\left(0.5-\delta_{\eta}\right)=$ $+\left(1-2 \delta_{\eta}\right) / 2$, the permanent electric dipole moment of a proton can be approximated similarly as

$$
\vec{d}_{\mathrm{p}} \approx \frac{e m_{h}\left(\alpha \pi_{C(\mathrm{p})}\right)}{2 m_{\mathrm{p}}} \hat{\mathbf{r}} \sim 5.419 \times 10^{-61} \mathrm{C} \mathrm{m} .
$$

Equation (97) can be modified for the case of a neutron as $\vec{d}_{\mathrm{n}} \approx \eta_{\mathrm{n}}\left(\alpha \pi_{C(\mathrm{n})}\right)\left(e / n_{\mathrm{n}}\right) \hat{\mathbf{r}}$. However, because $\eta_{\mathrm{n}} \approx 0$, it follows that $\left|\vec{d}_{\mathrm{n}}\right| \approx 0$, indicating that a neutron has no measurable permanent electric dipole moment.

\section{NUCLEAR FORCES AND ELEMENTARY PARTICLES}

This section describes the physical mechanism underlying the two central features of nuclear physics, namely (a) the forces that act to bind nucleons together in atomic nuclei and to pry them apart in nuclear decay, and (b) the formation and decay of elementary particles.

\section{A. Origin of nuclear forces}

As noted, the universal electrostatic force function given by Eq. (84) differs most notably from Coulomb's law in respect to the term $\mathcal{D}_{\eta}=\left(1-\bar{\rho}_{\odot} / \rho_{q}\right)$, which features prominently in the former function but does not appear in the latter. Furthermore, when $\bar{\rho}_{\odot}>\rho_{q}$, the negative value of $\mathcal{D}_{\eta}$ acts to invert the direction of the electrostatic force such that $\vec{F}_{\eta}$ becomes attractive between like charges and repulsive between unlike charges. Described below are the special conditions that exist inside atomic nuclei due to which $\vec{F}_{\eta}$ acts to bind together positively charged protons alongside electrically neutral neutrons to form nuclear clusters.

As described in Sec. IV D, a proton can be treated as a virtual single-cell particle with respect to its Compton wavelength, whereby from Eqs. (34) and (46) we have $\lambda_{C(\mathrm{p})}=2 L_{\mathrm{p}}$, where the polar chord $L_{\mathrm{p}}$ represents the approximate diameter of an average proton. Thus, if $\mathcal{R}_{\mathrm{p}}$ is the radius of an average proton, then it follows that $\lambda_{C(\mathrm{p})} \approx 4 \mathcal{R}_{\mathrm{p}}$. The mass density of an average proton $\grave{\rho}_{\mathrm{p}}$ can therefore be estimated as

$$
\begin{aligned}
\grave{\rho}_{\mathrm{p}} & =\frac{3 m_{\mathrm{p}}}{4 \pi \mathcal{R}_{\mathrm{p}}^{3}} \approx \frac{48 m_{\mathrm{p}}}{\pi \lambda_{C(\mathrm{p})}^{3}} \\
& \approx 1.10758 \times 10^{19} \mathrm{~kg} \mathrm{~m}^{-3} .
\end{aligned}
$$

Similarly, if $\mathcal{R}_{\mathrm{n}}$ is the radius of a neutron, then the mass density of an average neutron $\grave{\rho}_{\mathrm{n}}$ can be estimated as

$$
\begin{aligned}
\grave{\rho}_{\mathrm{n}} & =\frac{3 m_{\mathrm{n}}}{4 \pi \mathcal{R}_{\mathrm{n}}^{3}} \approx \frac{48 m_{\mathrm{n}}}{\pi \lambda_{C(\mathrm{n})}^{3}} \\
& \approx 1.113703 \times 10^{19} \mathrm{~kg} \mathrm{~m}^{-3} .
\end{aligned}
$$

Thus, the density of an average neutron lies just above that of an average proton in the density spectrum.

Neutrons are present along with protons in every atomic nucleus with the singular exception of the hydrogen-1 nucleus, which contains no neutrons. Suppose that a single proton constitutes a test charge $q_{0}^{+}$such that $\rho_{q}=\rho_{\mathrm{p}}$, and the test charge is located inside an atomic nucleus composed of multiple nucleons including protons as well as neutrons. The medium $\odot$ surrounding $q_{0}^{+}$then consists of both neutrons and protons whereby, given that 
$\rho_{\mathrm{n}}>\rho_{\mathrm{p}}$, the average density of the medium surrounding $q_{0}^{+}$must typically exceed that of the test charge such that $\bar{\rho}_{\odot}>\rho_{q}$. Consequently, pursuant to Eq. (84), the electrostatic force $\vec{F}_{\eta}$ between positively charged protons located inside an atomic nucleus becomes attractive if (and only if) the nucleus consists of a combination that includes neutrons in addition to protons.

Conventional particle theory invokes a distinct shortrange (attractive) strong nuclear force that acts to counter the (repulsive) Coulomb force $\vec{F}_{C}$ between positively charged protons inside an atomic nucleus. By contrast, the present model asserts that due to the special conditions that exist inside an atomic nucleus, it is the electrostatic force $\vec{F}_{\eta}$ itself that acts to keep positively charged protons along with electrically neutral neutrons bound together in an atomic nucleus. While every atomic nucleus with more than a single nucleon must contain neutrons along with protons for $\vec{F}_{\eta}$ to act as an attractive force, the stability of a nucleus further demands a critical balance between the numbers of protons and neutrons present therein.

In the present context where the test charge $q_{0}^{+}$is a single proton located inside an atomic nucleus, if the particles surrounding the test charge contain excessively many neutrons, then from Eq. (82) the total charge $Q$ can diminish considerably due to the presence of too many (electrically neutral) neutrons relative to the number of (positively charged) protons inside $\mathcal{S}$. Pursuant to Eq. (84), as the charge $Q$ diminishes in magnitude, so too does $\left|\vec{F}_{\eta}\right|$, reducing the strength of the (attractive) force that holds nucleons together inside a nucleus. In turn, the weakening of the attractive force between nucleons increases the susceptibility of the nucleus to disintegration from external stimuli, such as radiation energy received from an incident photon or kinetic energy from the impact of an incident moving particle.

Conversely, if the particles surrounding the test charge $q_{0}^{+}$inside a nucleus lack sufficiently many neutrons, then the condition $\bar{\rho}_{\odot}>\rho_{q}$ may not be satisfied, especially for those protons that are located on the surface of the nucleus. This is because, in addition to the neutrons and protons inside the nucleus, the neighbors of a proton located on the surface of an atomic nucleus include the inner electrons of the atom. In that case, the average ambient density $\bar{\rho}_{\odot}$ can become lower than $\rho_{q}$ if there is an insufficient number of (high-density) neutrons neighboring $q_{0}^{+}$to compensate for the diminution of $\bar{\rho}_{\odot}$ caused by the (comparatively low-density) inner electrons of the atom. Pursuant to Eq. (84), if $\bar{\rho}_{\odot}<\rho_{q}$ then $\vec{F}_{\eta}$ acts as a repulsive force between the positively charged protons, rendering the nucleus susceptible to disintegration (i.e., decay) due to the mutual repulsion of the protons present therein. According to this model, the neutron-to-proton ratio in the atomic nuclei of unstable

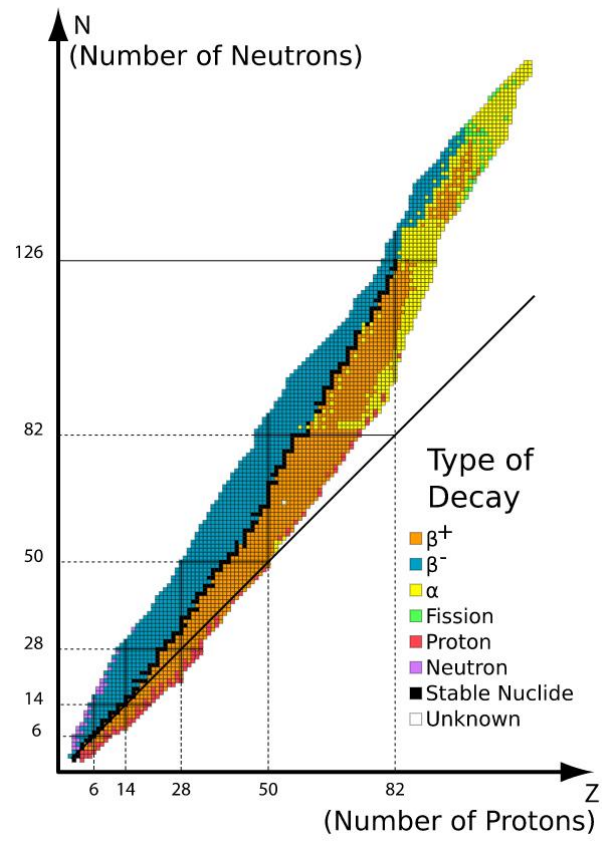

FIG. 9. Band of nuclear stability. Stable nuclides are shown in black, while unstable nuclides are color-coded to show the types of decay they tend to entail.

Source:

https://commons.wikimedia.org/wiki/File:Table_isotopes_en.svg

elements and isotopes is so precariously balanced that even minor environmental perturbations can cause the inversion of $\vec{F}_{\eta}$ from an attractive nuclear force to a repulsive one.

Therefore, as with the presence of excessively many neutrons, the absence of sufficiently many neutrons in an atomic nucleus also increases the susceptibility of the nucleus to disintegration from minor external stimuli. Conventional particle theory invokes a distinct shortrange (repulsive) weak nuclear force that acts to counter the (attractive) strong force between nucleons, thereby inducing nuclear decay. By contrast, the present model asserts that there is no nuclear force other than the universal electrostatic force as described by Eq. (84), which both binds nucleons together and pries them apart, depending on the extant conditions.

An atomic nucleus thus decays because it lacks the critical balance between the numbers of protons and neutrons present therein, where the balance is needed to satisfy the condition $\bar{\rho}_{\odot}>\rho_{q}$ for each proton in the nucleus, while maintaining a sufficiently high $Q$ value of the nucleus as a whole. The logic of this model implies that it is this critical balance, or absence thereof, in the neutron-to-proton ratios of the atomic nuclei of the various isotopes of known chemical elements that accounts for the observed shape and composition of the familiar band of nuclear stability (see Fig. 9). 


\section{B. Particle formation}

According to photodynamics, elementary particles are forged from the LDI cells of ordinary space through the process of phase transition (described in Sec. IV B) in conditions of extreme compression. Such conditions exist in the inner strata of an active star. The compression therein is sufficient to breach the lower elastic limits of LDI cells and trigger phase transitions, thus raising their rudimental densities progressively to LDT level, thereby creating electrons. Further compression triggers further phase transitions, raising the rudimental densities of LDT cells progressively to HDT level. The present model stipulates that this process is aided by convection currents that transport matter gradually from the core of an active star to its outer strata and vice versa. The convection thus causes low-density cells in the outer strata of the star to migrate to its inner strata and high-density cells to migrate from the inner strata to its outer strata. HDT cells that are transported to the outer strata of the star (i.e., to nonextreme environments) then cluster in characteristic concentric symmetry to form multi-cell nucleons. In this way, subatomic particles are continually created, altered and reprocessed over the active life of a star. The particles manufactured by this process are then ejected into the circumstellar medium partially during the lifetime of the star through periodic stellar flares, and entirely if the star explodes as a supernova at the end of its life.

As HDT cells cluster in nearest-density order around the highest-density cell in their vicinity to form a multicell particle, autonomic compulsions impose an added constraint on the evolution of the particle. As described by Eq. (89), $\sum_{i}\left( \pm R_{\eta, i}\right)$ must decrease in value with the incorporation of each additional cell to enforce the requisite reduction in the $\sum_{i} U_{\eta, i}$ of the evolving cluster. Consequently, the upper limit of the number of cells that a multi-cell HDT cluster can hold is reached when $\sum_{i}\left( \pm R_{\eta, i}\right) \simeq 0$. No further cells can then be added without causing the $\sum_{i} U_{\eta, i}$ of the cluster to increase in violation of the autonomic principle.

The final products of this process are multi-cell HDT clusters whose $\sum_{i}\left( \pm R_{\eta, i}\right) \simeq 0$, whereby their (net) energy imbalances $\sum_{i} \eta_{i}^{ \pm}$, and hence their net charges $\sum_{i} Q_{i}^{ \pm}$, must be as close as possible to zero. This implies that the fullyevolved HDT clusters must be neutrons carrying (approximately) zero charge. By the logic of this model, protons with unit positive charges are produced by the decay of neutrons, as described in Sec. VII C below.

(Note: As stated in Sec. III G, this mechanism permits the possibility that HDT cells first cluster in characteristic Gaussian symmetry to form sub- $C_{1}$ clusters as quarks, which, in turn, cluster in nearest-density order to form complete particles as neutrons and, following decay, protons. However, from the viewpoint of photodynamics, the inclusion of such sub- $C_{1}$ clusters in the theoretical construct of the model has no consequential bearing on any aspect of its broader logic.)

\section{Neutron decay}

As noted, the mechanism by which a multi-cell HDT particle is formed dictates that the $\eta$ values of the individual cells that make up the particle must be such that the cumulative $\eta$ of the developing cell cluster decreases maximally in value with each new addition of a cell. Moreover, each cell in the developing cluster must be arranged in nearest density order relative to its neighbors. Consequently, the internal structure of a multi-cell HDT particle entails an intricate and delicately balanced arrangement of cells, which can unravel under certain conditions. The decay of a multi-cell HDT particle (e.g., a neutron) is therefore described as the partial disintegration of the cellular structure of the particle, which can be triggered by any one of three events. These trigger events are (i) the absorption of radiation energy received via an incident photon, (ii) the absorption of kinetic energy received via the impact of a moving particle, and (iii) a reverse phase transition induced by extreme decompression.

If a cell that is located on the surface of a neutron receives photinos transmitted to it by an incident photon, then its photino content $H_{A}$ increases as it absorbs the incident photinos. In turn, the increase in $H_{A}$ increases the $V_{P}$ of the cell in accordance with Eq. (12), and if local spatial constraints prevent a commensurate increase in its $V_{A}$, then the cell acquires (reducible) potential energy $U_{V}$ in accordance with Eq. (13). If $\varphi$ is the photoelectric work function associated with the extant location of the cell, then as $U_{V} \rightrightarrows 0$, the cell gains kinetic energy $K=U_{V}-\varphi$ if $U_{V}>\varphi$. Consequently, the cell detaches from its host neutron and escapes, causing the cellular structure of the neutron to partially unravel, thus inducing its decay. The logic of photodynamics suggests that it is mainly this mechanism that is responsible for the spontaneous decay of neutrons inside atomic nuclei.

In the case where a surface cell of a multi-cell HDT particle receives kinetic energy imparted by an incident moving particle on impact, the kinetic energy received from the incident particle first converts into potential energy $U_{V}$ of the surface cell. Thereafter, the cellular structure of the multi-cell particle partially unravels through the same process as that in which the received energy is transmitted to the surface cell via an incident photon. The logic of the present model suggests that it is this mechanism that triggers the disintegration of nucleons in high-energy particle collisions.

As described in Sec. IV B, a reverse phase transition occurs when the upper elastic limit of a cell is breached such that $V_{A}>V_{P}$, which requires conditions of extreme 
decompression produced by the direct exposure of the cell to a sufficiently low-density ambient environment. The present model stipulates that no physical environment exists in nature where the density is so low that direct exposure to it can decompress a cell of proton density or a lower density (e.g., an electron) sufficiently to cause its upper elastic limit to be breached. This environmental threshold effectively establishes a mechanism akin to biological natural selection for elementary particles, whereby only those particles whose densities are below the threshold can survive direct exposure to every possible low-density environment in nature unaffected. A proton therefore does not decay because there is no environment in nature that can trigger a reverse phase transition in any of its constituent cells.

It follows that a reverse phase transition can occur in a cell if and only if its density is higher than that of a proton. As shown by Eqs. (101) and (102), the density of a neutron lies just above that of a proton in the density spectrum. Hence, a neutron can be described as a multicell HDT particle that contains cells whose densities render them susceptible to reverse phase transition under extreme decompression. In nature, the immediate aftermath of a supernova presents such conditions. As matter from an exploding star scatters into the circumstellar (LDI) medium, solitary neutrons and neutron clusters become exposed to a very low relative density environment and thereby to conditions of extreme decompression. Such conditions trigger a breach of the upper elastic limits of the highest-density cells located in the exposed surface of a neutron, causing their reverse phase transition and thereby a partial disintegration of the cellular structure of the neutron.

Whether the disintegration of a neutron occurs due to the energy received via an incident photon or a moving particle, or due to a reverse phase transition, its essential consequence is to pare the (unstable) neutron into a stable proton, while releasing additional daughter particles together with high-frequency photons. This process defines the mechanism of $\beta^{-}$neutron decay, as follows.

The difference between the total number of cells in an average neutron and an average proton can be calculated as $\left(n_{\mathrm{n}}-n_{\mathrm{p}}\right) / n_{\mathrm{e}} \approx 2.53098726$. However, because the number of cells in a multi-cell cluster must be an integer, the actual difference between the number of cells in a neutron and a proton must be $\left\lfloor\left(n_{\mathrm{n}}-n_{\mathrm{p}}\right) / n_{\mathrm{e}}\right\rfloor=2$. The fractional part of the previous value implies that, in addition to the photino contents of its two more cells, an average neutron contains roughly $\left(n_{\mathrm{e}} \times 0.53098726\right) \approx$ $6.56083 \times 10^{19}$ more photinos than does a proton, equivalent to roughly $4.34725 \times 10^{-14} \mathrm{~J}$ of energy.

It is well established that the decay of a free neutron by $\beta^{-}$emission leaves three particles, namely a proton, an electron and an antineutrino $(\bar{v})$, in addition to excess energy $\mathfrak{Q}$. Conventionally, the $\beta^{-}$decay of a neutron is expressed as

$$
{ }_{0}^{1} \mathrm{n}_{1} \rightarrow{ }_{1}^{1} \mathrm{p}_{0}+{ }_{-1}^{0} \mathrm{e}_{0}+\bar{v}+\mathfrak{Q} \text {. }
$$

This behavior is consistent with the results expected based on the logic of photodynamics with respect to the balance necessary for the conservation of energy, electric charge and the total number of particles in the system. As stated, the decay of a free neutron by $\beta^{-}$emission must produce one proton and precisely two unitary cells, as well as excess energy $\mathfrak{Q}$. Therefore, in addition to a proton with unit positive charge, one of the two single-cell daughter particles is an LDT cell (electron) with unit negative charge, while the other is an LDI cell $(\bar{v})$ with fractional charge. If prior to its decay the neutron carried a net zero electric charge, then charge is conserved if and only if cell $\bar{v}$ carries a (positive or negative) fractional charge such that its $\eta$ is equal to the (vanishingly small) numerical value of the net $\delta_{\eta}$ of the proton and the electron produced in the decay.

Note that because neutrinos and antineutrinos are LDI cells, their photino contents must typically be very small in comparison to cells that make up tangible matter. Given further that no cell can be devoid of photinos, it follows that neutrinos and antineutrinos cannot be massless, while their masses are determined by their extant photino contents in accordance with Eq. (5).

The excess energy $\mathfrak{Q}$ may be partly transmitted as the energy $E_{\gamma}$ carried by emitted photons and partly retained in the form of unbound photinos by the daughter particles, while the latter may generate potential energy $U_{V}$. Because $U_{V} \rightrightarrows 0$, motion may be induced in the daughter particles by the (partial or total) conversion of their $U_{V}$ into kinetic energy $K$. While the extant conditions specific to any given occurrence of $\beta^{-}$decay determine the proportion in which each form of excess energy may appear, if $\mathfrak{Q}$ is present in each of its forms, then its components are described by

$$
\mathfrak{Q}=E_{\gamma}+U_{V}+K+\varphi+n_{\text {ret }} h v_{0}
$$

where $n_{\text {ret }}$ is the total number of unbound photinos retained by the daughter particles.

\section{SCHRÖDINGER EQUATION, LINE SPECTRA, AND ATOMIC GEOMETRY}

\section{A. Schrödinger equation}

Photodynamics differs fundamentally from quantum mechanics in its characterization of the electron. The latter describes an electron essentially as a mathematical object of indeterminate physicality, while the location of the 
electron near an atomic nucleus must be deduced probabilistically. By contrast, photodynamics describes an electron as a unitary LDT cell and thus a physical particle with a well-defined spatial extent, whose location in an atom is determined by the universal mechanism of cell clustering. Electrons are thus arranged in characteristic concentric symmetry around an atomic nucleus.

If $\lambda_{n}$ is the wavelength of a stationary photino wave in mode $n$, then its associated energy $E$ is given by $E=$ $h c / \lambda_{n}$, which, based on Eq. (34), can be stated equivalently as $E=h c n / 2 L$. Squaring both sides of this equation and then multiplying the right-hand side by $\left(2 \pi^{2} m_{\mathrm{e}}\right) /\left(2 \pi^{2} m_{\mathrm{e}}\right)$, where $m_{\mathrm{e}}$ is the ground-state rest mass of an electron, yields the relationship

$$
E^{2}=\frac{\left(h^{2} \pi^{2}\right)\left(2 m_{\mathrm{e}} c^{2}\right) n^{2}}{\left(4 \pi^{2}\right) 2 m_{\mathrm{e}} L^{2}} .
$$

Let $E_{\mathrm{e}}$ denote the energy content of an electron in its ground state and let $E$ now denote the energy of its associated stationary photino wave in its fundamental mode with wavelength $\lambda_{1}$. Given that $\lambda_{1}=2 L$, where $L$ is the length of the polar chord of the electron, it follows that $E=2 E_{\mathrm{e}}=2 m_{\mathrm{e}} c^{2}$. Therefore, for $n=1$ and after some algebra, Eq. (105) reduces to

$$
E=\frac{\hbar^{2} \pi^{2}}{2 m_{\mathrm{e}} L^{2}} n^{2}
$$

where although unnecessary because $n^{2}=1$, the $n^{2}$ term is retained nevertheless for clarity and for consistency with the following derivations. Equation (106) can be stated equivalently as

$$
\frac{\hbar^{2}}{2 m_{\mathrm{e}}}=E\left(\frac{L^{2}}{n^{2} \pi^{2}}\right)
$$

Equation (35) gives the wave function of a stationary photino wave as $\psi(x) \approx \mathbb{A}_{x} \cos (n \pi x / L)$. Taking the second derivative of $\psi(x)$ with respect to $x$, we get

$$
\frac{\mathrm{d}^{2} \psi}{\mathrm{d} x^{2}} \approx-\left(\frac{\mathbb{A}_{x} n^{2} \pi^{2} \cos (n \pi x / L)}{L^{2}}\right)
$$

Dividing Eq. (35) by Eq. (108) thus yields

$$
-\frac{\psi(x)}{\left(\mathrm{d}^{2} \psi / \mathrm{d} x^{2}\right)} \approx \frac{L^{2}}{n^{2} \pi^{2}}
$$

Substituting the left-hand side of this equation into Eq. (107) gives $\hbar^{2} / 2 m_{\mathrm{e}} \approx-E\left(\psi(x) /\left(\mathrm{d}^{2} \psi / \mathrm{d} x^{2}\right)\right)$, which can be written as

$$
-\frac{\hbar^{2}}{2 m_{\mathrm{e}}} \frac{\mathrm{d}^{2} \psi}{\mathrm{d} x^{2}} \approx E \psi
$$

This function represents the one-dimensional case of a stationary photino wave whose axis of symmetry lies on the $x$-axis. Equation (110) can be extended to the case of multiple stationary photino waves whose respective axes of symmetry intersect at an origin (physically, a nucleus). For stationary photino waves in the same mode, each axis of symmetry has a different radial orientation from the origin such that the angle subtended at the origin is constant for each pair of nearest axes. This arrangement represents the three-dimensional case, described by the generalization of Eq. (110) as

$$
-\frac{\hbar^{2}}{2 m_{\mathrm{e}}}\left(\frac{\partial^{2} \psi}{\partial x^{2}}+\frac{\partial^{2} \psi}{\partial y^{2}}+\frac{\partial^{2} \psi}{\partial z^{2}}\right) \approx E \psi
$$

where $\psi$ is a function of $x, y$ and $z$. This equation describes the case in which the system is an atom in a state of equilibrium, in which every electron is in its ground state. The extant photino content of each electron $H_{A}$ is therefore equal to its ground-state photino content $H_{G}$. In addition, because the system is in equilibrium, the physical volume $V_{A}$ of each electron must be as close as possible to $V_{P}$, whereby $V_{A} \simeq V_{P}$. The total potential energy of each electron is therefore irreducible such that $U \simeq 0$ for every electron in the atom.

Now suppose that an electron absorbs photinos transmitted to it by an incident photon, raising its photino content such that $H_{A}>H_{G}$ and increasing its $V_{P}$ in accordance with Eq. (12). If spatial constraints in its extant location inhibit a corresponding increase in the $V_{A}$ of the electron, then $V_{P}>V_{A}$ and the electron acquires (reducible) potential energy $U>0$. Because Eq. (111) describes the case in which every electron in the atom is in equilibrium so that $U \simeq 0$, Eq. (111) must be modified to account for the case where an electron possesses potential energy $U \neq 0$, and stated accordingly as $-\hbar^{2} / 2 m_{\mathrm{e}}\left(\partial^{2} \psi / \partial x^{2}+\partial^{2} \psi / \partial y^{2}+\partial^{2} \psi / \partial z^{2}\right) \approx$ $(E-U) \psi$, which can be written equivalently as

$-\frac{\hbar^{2}}{2 m_{\mathrm{e}}}\left(\frac{\partial^{2} \psi}{\partial x^{2}}+\frac{\partial^{2} \psi}{\partial y^{2}}+\frac{\partial^{2} \psi}{\partial z^{2}}\right)+U(x, y, z) \psi \approx E \psi$

In the framework of quantum mechanics, Eq. (112) would represent an approximation of the time-independent Schrödinger equation. In the context of photodynamics, Eq. (112) gives the physical spatial coordinates of electrons located inside an atom and its energy levels, as shown below. 


\section{B. Atomic line spectra}

As described in Sec. VI A, the $\eta$ of an electron causes its center of mass to be displaced from the energy-neutral point $\vec{c}_{m \mid \eta=0}$ on its polar chord, which, in turn, induces electrostatic potential energy $U_{\eta}$. Moreover, from Eq. (63), $\mathcal{D}_{\eta}$ represents a normalized measure of the displacement of the center of mass of an electron from its equilibrium location within a host atom. The quantity $\mathcal{U}_{\eta}=U_{\eta} / \mathcal{D}_{\eta}$ therefore describes the electrostatic potential energy of an electron per unit displacement of its center of mass from the equilibrium location in its host atom. Based on Eq. (64) this relationship can be expressed as $\mathcal{U}_{\eta}=\left(n_{G} h v_{0} / 2\right)(\mathbb{d})^{2}$. From Eq. (5), $n_{G} h v_{0}=m_{G} c^{2}$, so the previous equation becomes $\mathcal{U}_{\eta}=\left(m_{G} c^{2} / 2\right)(\mathbb{d})^{2}$. In addition, from Eq. (62) we have $\mathbb{d}=R_{\eta} / \mathbb{r}$, whereby this function can be written as

$$
\mathcal{U}_{\eta}=\frac{1}{2} m_{G} c^{2}\left(\frac{R_{\eta(\mathrm{e})}}{\mathrm{r}_{\mathrm{e}}}\right)^{2}
$$

Because $\eta_{\mathrm{e}}$ lies in the cusp range, $R_{\eta(\mathrm{e})}$ must be (very nearly) maximal, which, pursuant to Eq. (79) implies that $R_{\eta(\mathrm{e})} \approx \alpha \lambda_{C(\mathrm{e})}$. Moreover, as $\mathbb{r}_{\mathrm{e}}=\lambda_{1} / 2 \pi$ by definition, Eq. (46) yields the relation $\mathrm{r}_{\mathrm{e}}=\lambda_{C(\mathrm{e})}$. Positing that the mode $n$ of the stationary photino wave of an electron inside an atom acts as a linear scaling factor on $\mathbb{r}_{\mathrm{e}}$ such that $\mathrm{r}_{\mathrm{e}, n}=n \mathrm{r}_{\mathrm{e}}$, Eq. (113) can be written to give $\mathcal{U}_{\eta}$ for any value of $n$ as $u_{\eta, n} \approx\left(m_{G} c^{2} / 2\right)\left(\alpha \lambda_{C(\mathrm{e})} / n \lambda_{C(\mathrm{e})}\right)^{2}$. Multiplying the right-hand side of this equation by $\left(\mathbb{k}_{e} / \mathbb{k}_{e}\right)^{2}$ yields $\mathcal{U}_{\eta, n} \approx\left(m_{G} c^{2} / 2\right)\left(\mathbb{k}_{e} / \mathbb{k}_{e}\right)^{2}(\alpha / n)^{2}$, which can be stated alternatively as

$$
\mathcal{U}_{\eta, n} \approx \frac{m_{G}}{2}\left(\frac{\alpha c}{\mathbb{k}_{e}}\right)^{2}\left(\mathbb{k}_{e}\right)^{2}\left(\frac{1}{n}\right)^{2}
$$

Notice that with the singular exception of the mode $n$, all arguments of this function have fixed values that cannot change under any nonextreme conditions. The numerical value of $\mathcal{U}_{\eta, n}$ therefore varies in response only to changes in the value of $n$. As shown in Sec. VI C, Coulomb's constant $\mathbb{k}_{e}=\alpha\left(\hbar c / e^{2}\right)=1 / 4 \pi \epsilon_{0}$. Replacing $\mathbb{k}_{e}$ accordingly, Eq. (114) can be stated as

$$
u_{\eta, n} \approx \frac{m_{G}}{2}\left(\frac{\alpha c e^{2}}{\alpha \hbar c}\right)^{2}\left(\frac{1}{4 \pi \epsilon_{0}}\right)^{2}\left(\frac{1}{n}\right)^{2}
$$

In the framework of photodynamics, the (quantized) energy levels $E_{n}$ of an atom are then given by the identity $E_{n}=-\mathcal{U}_{\eta, n}$. Replacing $\mathcal{U}_{\eta, n}$ in this equation with the right-hand side of Eq. (115) yields, on simplification, the Bohr formula describing electron energy levels associated with the emission and absorption spectra of the singleelectron hydrogen-1 atom, given as

$$
E_{n} \approx-\frac{m_{\mathrm{e}} e^{4}}{32 \pi^{2} \epsilon_{0}^{2} \hbar^{2}}\left(\frac{1}{n}\right)^{2},
$$

where the average ground-state electron mass $m_{\mathrm{e}}$ replaces the general ground-state mass term $m_{\mathrm{G}}$. Evaluating the constants in this equation gives the electron energy levels associated with the hydrogen line spectrum as

$$
E_{n} \approx-\frac{13.6}{n^{2}} \mathrm{eV},
$$

in agreement with empirical evidence, as well as with the energy levels predicted by the Bohr and quantum mechanical models. Hence, in its ground state $(n=1)$ the electron has energy $E_{1} \approx-13.6 \mathrm{eV}$, in the first excited state $(n=2)$ it has energy $E_{2} \approx-3.4 \mathrm{eV}$, while in the second excited state $(n=3)$ it has energy $E_{3} \approx-1.5 \mathrm{eV}$, and so on.

To account for the case of any one-electron atom (i.e., hydrogen-1 as well as ionized elements such as $\mathrm{He}^{+}, \mathrm{Li}^{++}$, and $\mathrm{Be}^{+++}$) the relation $\mathrm{r}_{\mathrm{e}, n}=n \mathrm{r}_{\mathrm{e}}$ has to be modified such that $\mathrm{r}_{\mathrm{e}, n}=(n / Z) \mathrm{r}_{\mathrm{e}}$, where $Z$ is the atomic number of the atom. Thus, for the general case of any one-electron atom, Eq. (117) becomes $E_{n} \approx-13.6\left(Z^{2} / n^{2}\right) \mathrm{eV}$.

Suppose that an electron located inside an atom is in an excited state such that $n>1$ and it possesses potential energy that does not exceed its photoelectric work function. Autonomic compulsions dictate that the electron must emit a photon to reduce its potential energy if the entropic condition given by Eq. (39) is satisfied, while the number of levels by which $n$ must decrease as a consequence of the photon emission is determined in accordance with Eq. (41). The energy $h v$ carried by the emitted photon is then the difference between the initial (higher) energy level of the electron $E_{\mathrm{I}}$ and its final (lower) energy level $E_{\mathrm{F}}$, whereby $h v=E_{\mathrm{I}}-E_{\mathrm{F}}$. Based on Eq. (116) and given that $h v=h c / \lambda$, after some algebra, the previous equation can be written equivalently as

$$
\frac{v}{c}=\frac{1}{\lambda} \approx \frac{m_{\mathrm{e}} e^{4}}{64 \pi^{3} \epsilon_{0}^{2} \hbar^{3} c}\left(\frac{1}{n_{\mathrm{F}}^{2}}-\frac{1}{n_{\mathrm{I}}^{2}}\right),
$$

where the numerical coefficient in this equation defines the Rydberg constant (denoted by $R_{\infty}$ ).

Because the autonomic process of energy transmission compels a cell to minimize its unbound photino count, every cell - including every electron inside an atom - tends normally to be in its ground state. Consequently, absorption spectra, which entail the transition of an electron from lower to higher energy levels, tend to be associated with cases where the electron 
is initially in its ground state such that $n=1$. Emission spectra, on the other hand, are not subject to this limitation because they entail transitions from higher to lower energy levels, implying that the electron must initially be in an excited state with any one of all possible $n$ values and may transition to any lower energy level down to $n=1$, as described previously in Sec. IV C.

\section{Quantum states and atomic geometry}

Equation (112) can be written in a more computationally tractable form in spherical coordinates as

$$
\begin{aligned}
-\frac{\hbar^{2}}{2 m_{\mathrm{e}}}\left(\frac{\partial^{2} \psi}{\partial r^{2}}\right. & +\frac{2}{r} \frac{\partial \psi}{\partial r}+\frac{1}{r^{2} \sin \theta} \frac{\partial}{\partial \theta}\left(\sin \theta \frac{\partial \psi}{\partial \theta}\right) \\
& \left.+\frac{1}{r^{2} \sin ^{2} \theta} \frac{\partial^{2} \psi}{\partial \phi^{2}}\right)+U(r, \theta, \phi) \psi \\
& \approx E \psi
\end{aligned}
$$

where $\psi=\psi(r, \theta, \phi)$. Using the standard technique of separation of variables, the solutions of Eq. (119) are restricted to those that are separable and can be factored as $\psi(r, \theta, \phi)=R(r) \Theta(\theta) \Phi(\phi)$, where $R(r), \Theta(\theta)$ and $\Phi(\phi)$ are all functions of single variables. The separated solutions of this function can then be expressed as

$$
\psi_{n, l, m_{l}}(r, \theta, \phi)=R_{n, l}(r) \Theta_{l, m_{l}}(\theta) \Phi_{m_{l}}(\phi)
$$

where $n=1,2,3, \ldots, \quad l=0,1,2, \ldots, n-1$ and $m_{l}=$ $0, \pm 1, \pm 2, \ldots, \pm l$. Notice that the separated solutions pertain specifically to the Laplacian component of Eq. (119), and can be viewed as generic mathematical representations associated now with purely imaginary standing waves. The interpretations of the indices $\left(n, l, m_{l}, m_{s}\right)$ in photodynamics differ profoundly from those in quantum theory. They describe not the quantum states of electrons, but rather parameters that describe the geometric coordinates of the electrons in an atom.

Because the axis of symmetry of each standing wave passes through the nucleus of an atom, each axis extends radially from the nucleus in two opposite directions. Consequently, half of the (maximum number of) electrons in an atom must lie in the northern hemisphere of the atom and the other half lie in its southern hemisphere. A fourth index is therefore needed to specify the hemisphere in which an electron is located, corresponding to the electron spin in quantum mechanics, namely $m_{s}= \pm(1 / 2)$.

To describe the physical location of an electron within an atom, its four associated indices $\left(n, l, m_{l}, m_{s}\right)$ are first converted into three parameters $(n, \ell, m)$ through the following transformations:

$$
n=n,
$$

$$
\ell=\left\{\begin{array}{ll}
l & \text { if } m_{s}=+\frac{1}{2} \\
(2 n-1)-l & \text { if } m_{s}=-\frac{1}{2}
\end{array},\right.
$$

and

$$
m=\left\{\begin{array}{ll}
m_{l} & \text { if } m_{l} \geq 0 \\
m_{l}+(2 l+1) & \text { if } m_{l}<0
\end{array} .\right.
$$

The spherical coordinates of the electron can then be obtained as

$$
\begin{gathered}
r=n, \\
\theta=\frac{\pi \ell}{2 n-1},
\end{gathered}
$$

and

$$
\phi=\frac{2 \pi m}{m_{\max }+1}
$$

where

$$
m_{\max }= \begin{cases}2 \ell & \text { if } \ell<n \\ 2((2 n-1)-\ell) & \text { if } \ell \geq n\end{cases}
$$

Analogous to geographic coordinates, the parameters $(n, \ell, m)$ describe the geometric coordinates of electrons in an atom. The $n$ coordinate corresponds to a discrete 'elevation' above the nucleus, and defines the shell number of an electron within its host atom. The $\ell$ coordinate corresponds to a 'latitude' measured relative to the north pole of the atom, and defines the subshell number of the electron within a shell. Meanwhile, the $m$ coordinate corresponds to a 'longitude' measured relative to a reference azimuth, and defines the ordinal number of the electron within a subshell.

The $n^{\text {th }}$ shell of an atom consists of a set of contiguous electrons such that each electron is centered on the outermost node of a standing wave that is in mode $n$ and extends radially from the nucleus (see Fig. 10). The case $n=1$ represents the innermost shell of the atom, while $n \in\{1,2,3, \ldots\}$. As a representation of atomic geometry, Eq. (119) describes the optimal packing of electrons in each shell of an atom.

The polar coordinate $\theta$ of an electron is measured relative to the polar axis of the atom (see Fig. 5). An equatorial $(X-Y)$ plane bisects the atom, dividing each shell into northern and southern halves. Each half-shell is segmented further into $n$ subshells such that each subshell lies on a plane parallel to the equatorial plane. The cases $\ell=0$ and $\ell=2 n-1$ denote the two subshells located at the northern and southern poles, respectively, of the shell, 


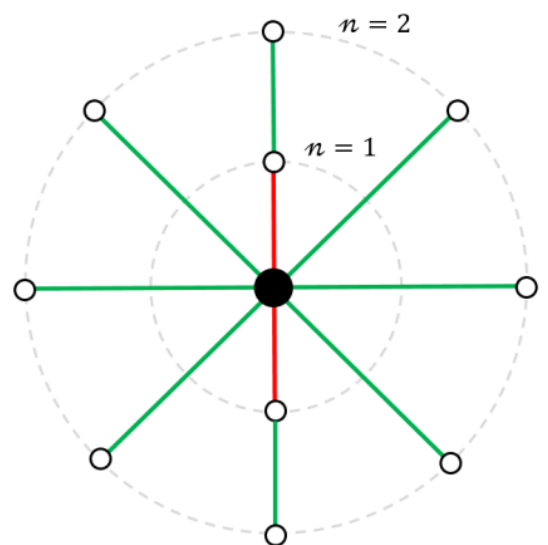

FIG. 10. Two-dimensional schematic of axes of symmetry of (imaginary) standing waves in modes 1 and 2. Red line: standing wave axis in mode $n=1$. Green lines: standing wave axes in mode $n=2$. Open circles: centers of mass of electrons. Filled-in circle: center of mass of nucleus.

while $\ell$ increases in the direction of increasing $\theta$, where $\ell \in\{0,1,2, \ldots, 2 n-1\}$. Within a given shell, each polar subshell consists of a unitary electron. Every other subshell contains multiple electrons, forming an ordered chain describing a circle centered about the polar axis of the atom (see Fig. 11).

Because the electrons in an atom must be arranged in nearest-density order, the highest-density electron within every subshell in an atom must lie in a common $Y-Z$ plane and on the same side of the polar axis of the atom. These features define the orientation and plane of the azimuth of an atom, forming the reference plane relative to which the azimuthal coordinate $\phi$ of an electron is measured. The case $m=0$ thus denotes the highest-density electron in a subshell, where $m \in\left\{0,1,2, \ldots, m_{\max }\right\}$ and $m$ increases in the direction of increasing $\phi$.

Table I gives the quantum states of electrons with the principal quantum numbers 1,2 and 3, alongside their corresponding spherical and geometric coordinates within a host atom. From the viewpoint of photodynamics, this precise correspondence implies that the quantum state of an electron - described equivalently by the electronic configuration given in spectroscopic notation - represents an allegorical depiction of the spatial coordinates of the electron in its host atom. Based on this logic, it can be further inferred that the electronic configurations of the chemical elements in general represent allegorical descriptions of the spatial coordinates of electrons located in the atoms of the respective elements.

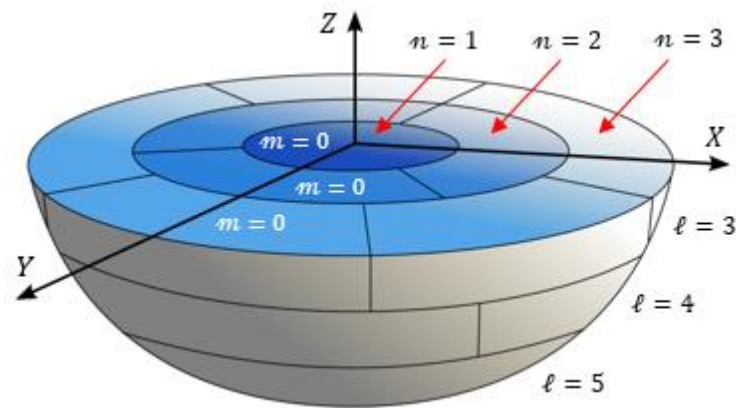

FIG. 11. Schematic of southern hemisphere of atom, showing three electron shells $n=1,2$ and 3. Subshells $\ell=3,4$ and 5 form part of the outer shell with $n=3$. The highest-density electrons in each subshell are aligned along the azimuthal $(Y)$ axis, each denoted by the ordinal number $m=0$.

\section{Heisenberg's uncertainty relation and Pauli's exclusion principle}

From classical wave mechanics, the inverse relationship between the spatial extent $\Delta x$ of the possible positions of a wave and the range of possible wave numbers $\Delta k$ within a wave packet composed of multiple superposed waves is stated as $\Delta x \Delta k \sim 1$. From the de Broglie model of the atom [35], we have $p=m c=h / \lambda=h k / 2 \pi=\hbar k$ and hence $\Delta k=\Delta p / \hbar$, whereby the Heisenberg uncertainty relation [11] is expressed as $\Delta x \Delta p \sim \hbar$. In addition, the uncertainty relation for the most compact distribution of wave numbers in the region $\Delta x$ is given by $\Delta x \Delta p=\hbar / 2$.

Differentiating $k=2 \pi / \lambda$ with respect to $\lambda$ and replacing the differentials with small intervals, the magnitude of $\Delta k$ can be derived to be $\Delta k=\left(2 \pi / \lambda^{2}\right) \Delta \lambda$. In the context of photodynamics, $\lambda$ describes the wavelength of the stationary photino wave in the fundamental mode $\lambda_{1}$, while the length $L$ of the polar chord of the associated electron in its ground state defines the maximum extent $\Delta x$ of the possible positions of all waves that (theoretically) could be superposed in the electron. Therefore, because $\Delta p=\hbar \Delta k$, it follows that $\Delta x \Delta p=L \hbar\left(2 \pi / \lambda_{1}^{2}\right) \Delta \lambda ;$ and as $L=\lambda_{1} / 2$ and $\Delta x \Delta p=$ $\hbar / 2$, the previous equation can be stated as $\left(\lambda_{1} \hbar / 2\right)\left(2 \pi / \lambda_{1}^{2}\right) \Delta \lambda=\hbar / 2$, which reduces to $\Delta \lambda=$ $\lambda_{1} / 2 \pi$. From Eq. (46), we have $\lambda_{1}=\lambda_{C}$, whereby the previous equation can be expressed equivalently as

$$
\Delta \lambda=\lambda_{C(\mathrm{e})}
$$


TABLE I. Spherical and geometric coordinates of electrons in shells 1, 2 and 3 according to their quantum states.

\begin{tabular}{|c|c|c|c|c|c|c|c|c|c|c|}
\hline \multirow[b]{2}{*}{$\begin{array}{c}\text { Principal } \\
\text { quantum } \\
\text { number } \\
n\end{array}$} & \multirow[b]{2}{*}{$\begin{array}{c}\text { Orbital } \\
\text { quantum } \\
\text { number } \\
l\end{array}$} & \multicolumn{3}{|c|}{ Quantum state } & \multicolumn{3}{|c|}{ Spherical coordinates } & \multicolumn{3}{|c|}{ Geometric coordinates } \\
\hline & & $\begin{array}{c}\text { Magnetic } \\
\text { quantum } \\
\text { number } \\
m_{l}\end{array}$ & $\begin{array}{c}\text { Spin } \\
\text { quantum } \\
\text { number } \\
m_{s}\end{array}$ & $\begin{array}{l}\text { Associated } \\
\text { spectroscopic } \\
\text { notation* }\end{array}$ & $\begin{array}{c}\text { Radial } \\
\text { coordinate } \\
r\end{array}$ & $\begin{array}{c}\text { Polar } \\
\text { angle } \\
\theta\end{array}$ & $\begin{array}{l}\text { Azimuthal } \\
\text { angle } \\
\phi\end{array}$ & $\begin{array}{c}\text { Shell } \\
\text { number } \\
n\end{array}$ & $\begin{array}{c}\text { Subshell } \\
\text { number } \\
\ell\end{array}$ & $\begin{array}{c}\text { Ordinal } \\
\text { number } \\
m\end{array}$ \\
\hline 1 & 0 & 0 & $+1 / 2$ & $1 s(1)$ & 1 & 0 & 0 & 1 & 0 & 0 \\
\hline 1 & 0 & 0 & $-1 / 2$ & $1 s(2)$ & 1 & $\pi$ & 0 & 1 & 1 & 0 \\
\hline 2 & 0 & 0 & $+1 / 2$ & $2 s(1)$ & 2 & 0 & 0 & 2 & 0 & 0 \\
\hline 2 & 0 & 0 & $-1 / 2$ & $2 s(2)$ & 2 & $\pi$ & 0 & 2 & 3 & 0 \\
\hline 2 & 1 & 0 & $+1 / 2$ & $2 p$ & 2 & $\pi / 3$ & 0 & 2 & 1 & 0 \\
\hline 2 & 1 & 1 & $+1 / 2$ & $2 p(2)$ & 2 & $\pi / 3$ & $2 \pi / 3$ & 2 & 1 & 1 \\
\hline 2 & 1 & -1 & $+1 / 2$ & $2 p$ & 2 & $\pi / 3$ & $4 \pi / 3$ & 2 & 1 & 2 \\
\hline 2 & 1 & 0 & $-1 / 2$ & $2 p$ (4) & 2 & $2 \pi / 3$ & 0 & 2 & 2 & 0 \\
\hline 2 & 1 & 1 & $-1 / 2$ & $2 p(5)$ & 2 & $2 \pi / 3$ & $2 \pi / 3$ & 2 & 2 & 1 \\
\hline 2 & 1 & -1 & $-1 / 2$ & $2 p(6)$ & 2 & $2 \pi / 3$ & $4 \pi / 3$ & 2 & 2 & 2 \\
\hline 3 & 0 & 0 & $+1 / 2$ & $3 s(1)$ & 3 & 0 & 0 & 3 & 0 & 0 \\
\hline 3 & 0 & 0 & $-1 / 2$ & $3 s(2)$ & 3 & $\pi$ & 0 & 3 & 5 & 0 \\
\hline 3 & 1 & 0 & $+1 / 2$ & $3 p(1)$ & 3 & $\pi / 5$ & 0 & 3 & 1 & 0 \\
\hline 3 & 1 & 1 & $+1 / 2$ & $3 p(2)$ & 3 & $\pi / 5$ & $2 \pi / 3$ & 3 & 1 & 1 \\
\hline 3 & 1 & -1 & $+1 / 2$ & $3 p(3)$ & 3 & $\pi / 5$ & $4 \pi / 3$ & 3 & 1 & 2 \\
\hline 3 & 1 & 0 & $-1 / 2$ & $3 p$ (4) & 3 & $4 \pi / 5$ & 0 & 3 & 4 & 0 \\
\hline 3 & 1 & 1 & $-1 / 2$ & $3 p(5)$ & 3 & $4 \pi / 5$ & $2 \pi / 3$ & 3 & 4 & 1 \\
\hline 3 & 1 & -1 & $-1 / 2$ & $3 p(6)$ & 3 & $4 \pi / 5$ & $4 \pi / 3$ & 3 & 4 & 2 \\
\hline 3 & 2 & 0 & $+1 / 2$ & $3 d(1)$ & 3 & $2 \pi / 5$ & 0 & 3 & 2 & 0 \\
\hline 3 & 2 & 1 & $+1 / 2$ & $3 d(2)$ & 3 & $2 \pi / 5$ & $2 \pi / 5$ & 3 & 2 & 1 \\
\hline 3 & 2 & 2 & $+1 / 2$ & $3 d(3)$ & 3 & $2 \pi / 5$ & $4 \pi / 5$ & 3 & 2 & 2 \\
\hline 3 & 2 & -2 & $+1 / 2$ & $3 d(4)$ & 3 & $2 \pi / 5$ & $6 \pi / 5$ & 3 & 2 & 3 \\
\hline 3 & 2 & -1 & $+1 / 2$ & $3 d(5)$ & 3 & $2 \pi / 5$ & $8 \pi / 5$ & 3 & 2 & 4 \\
\hline 3 & 2 & 0 & $-1 / 2$ & $3 d(6)$ & 3 & $3 \pi / 5$ & 0 & 3 & 3 & 0 \\
\hline 3 & 2 & 1 & $-1 / 2$ & $3 d(7)$ & 3 & $3 \pi / 5$ & $2 \pi / 5$ & 3 & 3 & 1 \\
\hline 3 & 2 & 2 & $-1 / 2$ & $3 d(8)$ & 3 & $3 \pi / 5$ & $4 \pi / 5$ & 3 & 3 & 2 \\
\hline 3 & 2 & -2 & $-1 / 2$ & $3 d(9)$ & 3 & $3 \pi / 5$ & $6 \pi / 5$ & 3 & 3 & 3 \\
\hline 3 & 2 & -1 & $-1 / 2$ & $3 d(10)$ & 3 & $3 \pi / 5$ & $8 \pi / 5$ & 3 & 3 & 4 \\
\hline
\end{tabular}

* By convention, the notation $n L^{N}$ describes the number $N$ of electrons for any given value of $L$ (symbolically named $s, p, d, f, \ldots$ connoting $l$ values $0,1,2,3, \ldots$ respectively) in each $n$ value of an atom. To allow all possible electrons for each $n$ value to be listed individually, the superscript $N$ is replaced in this table by the sequential order (shown in brackets) in which electron orbitals are typically filled for each $l$ value pursuant to Hund's rule.

Therefore, from the viewpoint of photodynamics, the Heisenberg uncertainty relation is, in essence, a statement to the effect that if the stationary photino wave of an electron were to consist of multiple superposed waves, then the minimum uncertainty in wavelength, $\Delta \lambda$, would be given precisely by $\lambda_{C(\mathrm{e})}$. However, given that the present model entails no assumptions in respect of wave superposition to account for the wavelength and form of the stationary photino wave, any formulation of uncertainty in this regard is rendered superfluous.

Unlike the abstract description of the electron in quantum mechanics, the present model describes an electron as a corpuscular single-cell object with a physical volume $V_{A}>0$. From the perspective of photodynamics, therefore, Pauli's exclusion principle [12] describes the truism that the same volume of space cannot be occupied simultaneously by more than one physical object.

\section{E. Double-slit experiment}

Photodynamics offers a straightforward rationale for the otherwise mysterious phenomenon associated with the well-known double-slit experiment [36]. In this experiment, atomic-scale particles such as electrons or protons are fired one at a time through two narrow parallel slits in a screen and are detected as they strike a second screen on the opposite side. As the number of particles sent through the two slits increases, a pattern of bright and dark bands is observed to build up on the rear screen. However, such a pattern can only be due to interference caused by the superposition of overlapping waves. Because ordinary physical bodies do not superpose, this phenomenon implies an abstract duality whereby atomicscale particles appear to behave also as waves.

According to the present model, this phenomenon is caused not by a subliminal wave-particle duality in the (quantum mechanical) character of atomic-scale particles but rather by the dynamical behavior of the LDI medium through which the particles move. A minor compression wave is produced in the medium when an electron gun fires an electron. The compression wave propagates through the medium to the first screen and through the two slits to the rear screen. However, it diffracts as it passes through the slits. The points at which the two diffracted 
wavefronts overlap form the locus of constructive interference, so the density of the medium is maximal at the locus. An electron is of higher density than the LDI cells in the medium, whereby its equilibrium location at any given instant is with those cells in the medium whose densities are the closest to its own. These are the maximally dense cells at the locus of each wavefront. In effect, the electron 'surfs' the shifting locus of constructive interference from the front screen to the rear screen. It therefore illuminates the rear screen at the point at which its carrier locus strikes that screen. This process repeats each time an electron is fired, causing the rear screen to be illuminated at each point at which a locus of constructive interference strikes it. Because the spaces between the loci are left unilluminated, the bright and dark bands characteristic of an interference pattern appear after multiple electrons have been registered on the rear screen.

The interference pattern produced in the double-slit experiment in which monochromatic light passes through two nearby slits can be explained similarly. However, the wavefront in this case is of photinos conducted from cell to cell in the LDI medium. Therefore, the concentration of photinos is maximal at the locus of constructive interference where the diffracted wavefronts emerging from the two slits overlap. As the loci carrying the highest concentration of photinos reach the rear screen, they illuminate it the most, while the lower concentrations of photinos in the spaces between the loci illuminate the screen less, thereby producing a pattern of bright and dark bands characteristic of wave interference.

\section{GRAVITATION}

\section{A. Hierarchy of cosmic clusters}

Further to the discussion on the hierarchy of nested clusters in Sec III G, all clusters of ranks higher than $C_{4}$ (i.e., molecules) represent celestial bodies and higher order systems. A $C_{5}$ cluster is thus defined as a planet (e.g., Earth) or a moon or another body of matter that is located within a system dominated by a local star (e.g., the solar system). A $C_{5}$ cluster typically consists of various substances, each of which is composed of numerous $C_{4}$ systems arranged (at the strictly local level) in nearestdensity order.

For the purposes of the discussion to follow, the intangible region of space separating the surface of a tangible body of matter that forms the core of a cluster and the outer boundary of the cluster is defined herein as the dominion of the core body. In effect, a dominion can be regarded as the region of intangible space in which the influence of the core body predominates such that all proper elements of the medium inside the dominion are in equilibrium when arranged in order of nearest density around the core body. For instance, the dominion of Earth can be described roughly as the region of space extending from the edge of the upper atmosphere to points beyond the outer bounds of the terrestrial magnetosphere.

A distinction must also be made between the term 'cluster' in its generic sense and in the sense of a distinct object within the present context. A unicluster is hence defined herein as a unitary body that includes both components of a well-ordered cluster, namely the core body of tangible matter that lies at the center of mass of the cluster and the dominion that surrounds the core body. A unicluster is thus a distinct physical object within its ambient medium and represents a single proper element of the higher-order host system in which it resides.

A $C_{6}$ cluster is defined as the complete unicluster of a $C_{5}$ cluster, and resides as a single unitary body within the dominion of a stellar body. For instance, the terrestrial unicluster includes the Earth and the terrestrial dominion, while the terrestrial unicluster resides as a unitary object within the solar dominion.

A $C_{7}$ cluster is defined as the unicluster of a stellar body, which includes the body proper as well as its dominion. For instance, the solar unicluster consists of the Sun at its core and the solar dominion that surrounds it, where the latter occupies the region of space extending from the solar corona to points beyond the outer bounds of the heliosphere. $C_{7}$ uniclusters typically reside within the dominions of galaxies. However, lone $C_{7}$ uniclusters and small groups of $C_{7}$ uniclusters may also be located in otherwise unpopulated regions of intergalactic space, as long as they satisfy the equilibrium condition (described in Sec. IX D below).

A $C_{8}$ cluster is defined as the complete unicluster of a galaxy. It consists of the galactic core, together with its dominion extending to points beyond the outer bounds of the surrounding dark halo, including all stellar uniclusters and other bodies of matter present therein.

In turn, autonomic compulsions induce $C_{8}$ uniclusters to form $C_{9}$ groupings as galactic clusters and superclusters. From the perspective of the higher-order $\left(C_{10}\right)$ host system, every $C_{9}$ grouping in it is just another proper element that, despite its vast scale, is indistinct from any of its other proper elements except in respect of its density.

The universe as a whole is defined as a $C_{10}$ cluster, within which the distribution of matter at all cosmic scales is described by the ubiquitous pattern of nested uniclusters detailed above. If this pattern also holds true at the $C_{10}$ level, then the complete universe may itself be a unicluster in which the observable universe represents the core, and a grand dominion composed of an LDI medium extends far beyond the outer edges of the observable core.

\section{B. Gravitational mechanics}

Based on Eq. (33), a satellite body (denoted by $\ominus$ ) located in the dominion of a primary body $\oplus$ is in equilibrium with 
respect to its ambient medium when $\left(\rho_{\ominus}-\bar{\rho}_{\odot}\right) \simeq 0$. A normalized measure of the displacement from equilibrium, $\mathcal{D}_{g}$, of a satellite body with respect to its ambient medium can be defined as

$$
\mathcal{D}_{g}=\left(\frac{\rho_{\ominus}-\bar{\rho}_{\odot}}{\rho_{\ominus}}\right)=\left(1-\frac{\bar{\rho}_{\odot}}{\rho_{\ominus}}\right) .
$$

If $U_{g}$ is the potential energy induced in a satellite body of mass $m_{\ominus}$ by its displacement from equilibrium within the dominion of $\oplus$, then a scale-normalized measure of $U_{g}$ can be defined as the gravitational potential $\mathcal{U}_{g}$ such that $\mathcal{U}_{g} \triangleq U_{g} / m_{\ominus}$. As described in Sec. III E, an approximately Gaussian structure emerges inevitably in the density distribution of proper elements in the system surrounding a primary body as the system approaches equilibrium. Let $\mathcal{S}$ be an imaginary spherical surface that is centered on $\oplus$ and lies within its dominion. If a satellite body of mass $m_{\ominus}$ and density $\rho_{\ominus}$ is located such that $\mathcal{S}$ passes through its center of mass, then pursuant to Eq. (14), the displacement of $\ominus$ from its equilibrium location induces a gravitational potential $\mathcal{U}_{g}$, given as

$$
\mathcal{U}_{g}=\frac{1}{2} k_{g} \mathcal{D}_{g}^{2}=\frac{1}{2} k_{g}\left(1-\frac{\bar{\rho}_{\odot}}{\rho_{\ominus}}\right)^{2}
$$

Positing that the gravitational potential at any given point inside a system is greater if its elements are packed tightly together than if they are packed loosely, it can be deduced that the coefficient $k_{g}$ varies with the compactness $p_{\mathcal{S}}$ of the system enclosed in $\mathcal{S}$ such that

$$
k_{g} \propto p_{\mathcal{S}} .
$$

Based on Eq. (31), this relationship can be written as $k_{g} \propto$ $m_{\mathcal{S}}\left(A_{\mathcal{S}} / V_{\mathcal{S}}\right)$ or equivalently as $k_{g} \propto\left(3 m_{\mathcal{S}} / r\right)$, where $r$ is the distance between the centers of mass of $\oplus$ and $\ominus$, and $m_{\mathcal{S}}$ is the sum of the masses of all the proper elements in the system enclosed by $\mathcal{S}$, namely the primary body, the LDI medium in its dominion, and every other body of matter present therein. If the proportionality coefficient associated with this relation is $G / 3$, where $G \approx 6.674 \times$ $10^{-11} \mathrm{~m}^{3} \mathrm{~kg}^{-1} \mathrm{~s}^{-2}$ is the universal gravitational constant, then it follows that

$$
k_{g}=G \frac{m_{\mathcal{S}}}{r} .
$$

Pursuant to Eq. (30), the medium in the dominion of $\oplus$ has an appreciable density gradient only in the radial direction. Consequently, the equilibrium location of $\ominus$ must be at a point that lies on the axis of $r$. A vector $\vec{D}_{g}$ can be defined to describe the magnitude and direction of the dislocation of $\ominus$ from its equilibrium point, as a proportion of its radial distance $r$ from $\oplus$, such that

$$
\overrightarrow{\mathrm{D}}_{g}=\left(\frac{\mathcal{D}_{g}}{r}\right) \hat{\mathbf{r}}=\frac{1}{r}\left(1-\frac{\bar{\rho}_{\odot}}{\rho_{\ominus}}\right) \hat{\mathbf{r}},
$$

where $\hat{\mathbf{r}}$ is outwardly normal to $\mathcal{S}$. This equation implies that the equilibrium point of $\ominus$ is its extant location on the axis of $r$ if and only if $\rho_{\ominus} \simeq \bar{\rho}_{\odot}$. However, if $\bar{\rho}_{\odot}<\rho_{\ominus}$, then $\ominus$ is dislocated from equilibrium in the direction of $\hat{\mathbf{r}}$ such that its equilibrium point lies closer to $\oplus$ along the axis of $r$ than does its extant location. Conversely, if $\bar{\rho}_{\odot}>$ $\rho_{\ominus}$, then $\ominus$ is dislocated from equilibrium in the direction antiparallel to $\hat{\mathbf{r}}$ such that its equilibrium point lies farther from $\oplus$ along the axis of $r$ than does its extant location.

According to Eq. (16), the potential energy induced by the dislocation of a body from its equilibrium point produces a restoring force proportional to the dislocation, directed antiparallel to the direction of the dislocation. If $\vec{F}_{g}$ is the restoring force acting on $\ominus$, then a scalenormalized measure of $\vec{F}_{g}$ can be defined as the gravitational field $\vec{g}$ such that $\vec{g} \triangleq \vec{F}_{g} / m_{\ominus}$. It follows that the gravitational potential $\mathcal{U}_{g}$ at any given point on $\delta$ produces a restoring force per unit mass, described by $\vec{g}$ at that point and stated as

$$
\vec{g}=-k_{g} \overrightarrow{\mathrm{D}}_{g}=-G\left(\frac{m_{\delta}}{r^{2}}\right)\left(1-\frac{\bar{\rho}_{\odot}}{\rho_{\ominus}}\right) \hat{\mathbf{r}}
$$

which can be expressed equivalently as

$$
\vec{F}_{g}=-G \frac{m_{\mathcal{\delta}} m_{\ominus}}{r^{2}}\left(1-\frac{\bar{\rho}_{\odot}}{\rho_{\ominus}}\right) \hat{\mathbf{r}}
$$

This equation describes the gravitational force $\vec{F}_{g}$ between a satellite body of mass $m_{\ominus}$ and a primary body of mass $m_{\oplus}$, such that the total mass $m_{\mathcal{S}}$ enclosed in $\mathcal{S}$ is given by

$$
m_{\mathcal{S}}=m_{\oplus}+m_{\odot},
$$

where $m_{\odot}$ is the total mass of all the proper elements of the medium enclosed in $\mathcal{S}$.

In both Newton's model of gravity and Einstein's general theory of relativity, the medium of space surrounding a primary body is assumed, tacitly if not explicitly, to be an empty vacuum. In the context of photodynamics, an empty vacuum as the medium of the dominion of a primary body implies that $m_{\odot}=0$ and $\bar{\rho}_{\odot}=0$ everywhere. It follows that when $m_{\odot}=\bar{\rho}_{\odot}=0$, Eq. (136) becomes $m_{\mathcal{S}}=m_{\oplus}$ and $\left(1-\left(\bar{\rho}_{\odot} / \rho_{\ominus}\right)\right) \hat{\mathbf{r}}=\hat{\mathbf{r}}$, whereby Eq. (135) reduces to Newton's law of gravity 


$$
\overrightarrow{\mathbb{F}}_{\mathrm{N}}=-G \frac{m_{\oplus} m_{\ominus}}{r^{2}} \hat{\mathbf{r}}
$$

where $\vec{F}_{N}$ denotes the gravitational force according to Newton's law. Thus, Newton's law of gravity represents a limiting case of Eq. (135) when space is assumed to be an empty vacuum devoid of any material content.

Contrary to conventional theory, Eq. (135) asserts that gravity is not a unidirectional force. If $\bar{\rho}_{\odot}<\rho_{\ominus}$, then $\vec{F}_{g}$ describes an attractive force, in agreement with the conventional view of gravity. However, if $\bar{\rho}_{\odot}>\rho_{\ominus}$, then $\vec{F}_{g}$ becomes a repulsive force, which in conventional theory is deemed a distinct phenomenon described as the buoyancy force produced by the net pressure $P$ exerted on a body by the medium in which it is submerged. From the perspective of photodynamics, conventional theory presents an inverted view of the direction of causation with respect to the phenomenon of buoyancy. This is because it is not the net pressure exerted by the ambient medium that produces an outward (buoyancy) force on the submerged body. Rather, it is the force $\vec{F}_{g}$ that produces the (net) outward pressure $P$ on the body when $\bar{\rho}_{\odot}>\rho_{\ominus}$, as the formulations that follow illustrate.

Pursuant to the definition given above, the magnitude of the gravitational field at any point in the dominion of $\oplus$ is given by $g=F_{g} / m_{\ominus}$. Dividing both sides of this equation by the compactness of $\ominus$ yields $g / p_{\ominus}=$ $F_{g} / m_{\ominus} p_{\ominus}=\left(F_{g} / m_{\ominus}^{2}\right)\left(V_{\ominus} / A_{\ominus}\right)$, which can be stated equivalently as $g / p_{\ominus}=\left(F_{g} / A_{\ominus}\right)\left(1 / m_{\ominus} \grave{\rho}_{\ominus}\right)$, where $\grave{\rho}_{\ominus}$ is the mass density of $\ominus$. Given that the pressure $P$ exerted on $\ominus$ by the ambient medium is described by $P=F_{g} / A_{\ominus}$, the previous equation can be written as $g / p_{\ominus}=P / m_{\ominus} \grave{\rho}_{\ominus}$, which can be expressed alternatively as

$$
P=m_{\ominus} g\left(\frac{\grave{\rho}_{\ominus}}{p_{\ominus}}\right)=F_{g}\left(\frac{\grave{\rho}_{\ominus}}{p_{\ominus}}\right)
$$

As this formulation shows, $P$ is a function of $F_{g}$, whereas $F_{g}$ derives from first principles defined by the theoretical foundations of photodynamics, as described above. Hence, in the context of the present model, the direction of causation must be from $F_{g}$ to $P$.

No fundamental distinction can therefore be drawn between the forces of gravity and buoyancy other than in their respective (antiparallel) orientations along the axis of $r$; both arise from a shared dynamic summarized by Eq. (135). Moreover, Eq. (135) holds true for any object located at any point in the space separating the center of mass of a primary body and the outer boundary of its dominion, including points inside the primary body. For instance, a point deep in the ocean can be considered a shallow location inside the Earth. Equation (135) thus applies as much to an air bubble released at the bottom of the Mariana Trench as to a pebble released midair by a child in a playground.

\section{Dark matter}

In the framework of photodynamics, the medium of space surrounding a primary celestial body $\oplus$ such as the Sun consists predominantly of LDI cells together with proportionately minor quantities of microscopic particles such as gas molecules, free atoms and ions. Also sparsely populating the dominion of a large primary body are such satellite bodies as planets, their moons, asteroids and comets, whose individual masses tend to be insignificant compared to the mass of the primary body. Consequently, when $\mathcal{S}$ encloses the inner region of the dominion of $\oplus$, the total mass of the medium enclosed in $\mathcal{S}$ is proportionately negligible, rendering $m_{\odot} \ll m_{\oplus}$ and hence $m_{\mathcal{S}} \approx m_{\oplus}$, whereby Newton's gravitational law holds true for all practical purposes. However, because the volume of space enclosed in $\mathcal{S}$ increases with its radius at a rate proportional to $r^{3}$, at greater distances from $\oplus, m_{\odot}$ no longer remains negligible compared to $m_{\oplus}$, rendering $m_{\mathcal{S}} \approx m_{\oplus}$. The Newtonian model thus fails to describe accurately the gravitational behavior of celestial objects whose distances from the primary body of their host unicluster are so large that $m_{\mathcal{S}}$ differs considerably in value from $m_{\oplus}$.

By definition, the value of $r$ is larger if $\mathcal{S}$ is a closed surface in the outer region of a large-scale system than in the outer region of a smaller system. Consequently, the divergence between $m_{\mathcal{S}}$ and $m_{\oplus}$ must be greater in the periphery of large-scale systems than in the periphery of smaller systems. A pronounced deviation is therefore seen in the gravitational behavior, such as the orbital periods, of celestial bodies located in the periphery of large-scale cosmic systems (e.g., galaxies and galactic clusters) from their behavior as predicted by the Newtonian model [37].

The standard model of cosmology attributes this deviation to the hypothesized presence of mass associated with unknown bodies of so-called dark matter that pervade the universe $[19,20]$. By contrast, the present model asserts that the vast expanses of seemingly empty space across the cosmos consist of contiguous massbearing cells as their elemental constituents, whereby $m_{\odot}$ must be appreciable in magnitude at points located in the outer reaches of large-scale cosmic systems. Thus, according to photodynamics, the missing mass attributed to bodies of dark matter in conventional theory is simply the collective mass of the LDI cells that make up the intangible medium spanning the vast expanses of seemingly empty space across the cosmos. 


\section{Equilibrium condition in cosmic systems}

Equation (33) asserts that a cosmic unicluster is in equilibrium in its extant location if and only if its density is as close as possible to the average density of the medium ○ that surrounds the unicluster. However, given that the density of the core body $\ominus$ in any cosmic unicluster is typically much higher than that of the medium $\odot$ that surrounds the unicluster, Eq. (33) can be satisfied if and only if the dominion of $\ominus$ has a lower density than $\odot$. (Note: The term core body refers herein to the core of a cosmic unicluster of any rank, where the core may consist of a single celestial body or a group of lower-order uniclusters collectively.)

The question then arises as to what the mechanism might be that lowers the density of the dominion of a core body such that its unicluster attains equilibrium in a location far from the primary body in its host system. While it must be left to future research to ascertain its validity, the logic of the present model suggests that the densities of the dominions inside cosmic uniclusters may be lowered preternaturally through the partial inversion of their natural (roughly Gaussian) density structures. This model stipulates that much like the photino density distribution inside a cell, the density distribution of the medium surrounding the core body inside a cosmic unicluster is determined substantially by the ever-present anisotropy in the density distribution of the medium surrounding the unicluster (see Sec. III E).

In this model of celestial dynamics, the dominion of a core body $\ominus$ is vastly greater in volume than the core body, extending (by way of comparison) to points beyond the farthest reaches of the magnetic influence of the core body (e.g., the magnetosphere of a planet or the heliosphere around the solar system). Furthermore, the dominion of $\ominus$ (and hence its unicluster) is invariably ovoid; compressed and densified on the forward side facing the direction of increasing ambient density, and elongated and rarefied on the aft side facing the direction of decreasing ambient density. This divergence in the shape of a dominion from its characteristic, roughly spherical Gaussian form is stipulated to be due fundamentally to the anisotropy in the density distribution of the (external) medium ๑ surrounding the unicluster, as follows.

As the cells in the outer boundary layer of the dominion of $\ominus$ are compelled to cluster in nearest-density order with the cells in the external medium $\odot$, the boundary layer of the dominion of $\ominus$ inevitably traces the (anisotropic) density contours of $\odot$. The shape of the dominion, and hence the unicluster, thus reflects the higher ambient density on its forward side and the progressively lower ambient density in the direction of its aft side. Meanwhile, the density of the medium within the elongated aft-side of the dominion decreases progressively inwards from the boundary region, whereas the density of the medium in the near vicinity of the core body $\ominus$ decreases in the (characteristic) radially outward direction. Thus, the density of the medium inside the dominion of $\ominus$ reaches its minimum value in the aft side of the dominion in regions occupied by its tail lobes corresponding to the tail lobes that can be detected inside the elongated tails of magnetic bubbles around celestial bodies [38]. This structure of spatial density inside the dominion of the core body in a cosmic unicluster can be described as a preternatural partial inversion of the characteristic Gaussian structure expected in the internal density distribution of a dominion.

According to this model, the tail lobes in the dominion of a core body are the lowest-density regions in a cosmic unicluster, while the density of the medium inside the tail lobes is lower than the average density of the external medium $\odot$. Moreover, the tail lobes occupy most of the total volume of the (elongated) aft side of the dominion of $\ominus$, and are vastly greater in volume than the core body $\ominus$ itself. Because of their great volume and ultralow (relative) density, the tail lobes act to lower the density of the dominion to well below that of $\odot$, thereby lowering the density of the complete unicluster to a level far below that of the body $\ominus$ that lies at its core. Thus, in accordance with Eq. (33), a cosmic unicluster attains equilibrium within its higher-order host system when the density of the unicluster as a whole is as close as possible to the density of its ambient medium.

If this model of celestial dynamics is correct, then it implies that such a partially inverted density structure must not only be a ubiquitous feature of dominions in cosmic uniclusters, but must also be a vital factor in determining the orbital distances of celestial bodies relative to the primary bodies in their respective host systems. It further implies that no cosmic unicluster can long survive as a satellite system at a stable orbital distance from the primary body in its host system if the internal density structure of its dominion fails to partially invert in this way.

\section{E. Dark energy}

As noted, if the above-described pattern of nested uniclusters also holds true at the $C_{10}$ level, then the complete universe may itself be a grand unicluster, of which the observable universe is the core and a vast surrounding region composed of an LDI medium constitutes the dominion. If this is so, then it further suggests that the unicluster of our universe may be bounded, implying that it may lie within a higher-order host system as one amongst numerous similar $C_{10}$ uniclusters. In that case, periodic variations in the conditions prevailing in its external environment should subject the unicluster of our universe to cycles of compression and decompression that, in turn, produce alternating phases of contraction and dilation in our universe. Observational evidence indicates that the 
universe is currently expanding, which suggests that the present epoch may be part of a dilation phase.

The contemporary standard cosmological model hypothesizes the existence of an unknown form of energy, namely dark energy [21], that pervades our universe and acts as a source of negative pressure that counters the gravitational self-attraction of matter to produce the observed expansion. Contrary to this view, the present model suggests that the expansion of the universe may be caused not by any internal factor such as dark energy, but rather by the decompression of the unicluster of our universe induced by the (external) conditions prevailing in its ambient system.

\section{DISCUSSION AND CONCLUSION}

Does the ability of a physical theory to make predictions that agree with scientific observations constitute sufficient reason to conclude that the precepts upon which the theory is built must necessarily be true? This essential question has been explored in this paper through the reconstruction of an array of time-tested equations of conventional theories based on principles that differ fundamentally from those upon which the conventional theories are built. Thus, the implicit argument being made is that if these equations, and thereby their predictive authority, can be reproduced by an alternative theory based on unconventional precepts, then it follows that the precepts underlying the conventional theory are unexceptional and can, in principle, be altered without diminishing the predictive power of the theory.

The development of physics as a discipline over the past four centuries has occurred through the study of different aspects of nature individually, whereby largely isolated domains of physics describe distinct aspects in nature with few explicit links connecting the domains to one another. By contrast, the model that has been presented in this paper is based on a foundation of shared principles within a framework of relational causality. The aspects that have been shown to emerge from these principles include those described by such fundamental domains of conventional theory as electromagnetism, quantum mechanics, gravitation, particle physics, optics, and thermodynamics.

Key mathematical relationships that have been reconstructed rigorously within this theoretical framework include the Schrödinger equation, Bohr's formula describing the hydrogen spectrum, Newton's gravitational law, Coulomb's law of electrostatic force, formulae describing the Compton effect, formula relating the wavelength of light to the refractive index of a medium, and formulae describing electric and magnetic fields. Newton's law of gravity has been shown to derive from a more complete universal gravitational force function, where the former is a limiting case of the latter when the medium of space is assumed to be an absolute vacuum. The quantum-mechanical formulation of the Schrödinger equation has been shown to be a mathematically precise allegorical depiction of the geometric distribution of energy associated with unequivocally physical electrons arranged symmetrically around an atomic nucleus.

Electric charge has been shown to be an emergent feature that is produced inevitably by the internal structure of elementary particles. Coulomb's law has been shown to be a limiting case of a more complete universal electrostatic force function, where the latter has also been shown to describe the nuclear forces that act to both bind nucleons together in atomic nuclei and pry them apart in nuclear decay.

Also described are some important implications of the proposed model. For instance, what is hypothesized in the standard model of cosmology to be the mass associated with unknown bodies of dark matter has been shown to be the cumulative mass of an ever-present material content that subsists in the fabric of space everywhere. In atomic theory, the measurement problem associated with the quantum-mechanical description of the wave function does not arise in the proposed model because it describes the wave function explicitly as a well-defined representation of the internal composition of a physical particle. Meanwhile, a simple deterministic mechanism has been shown to describe the characteristic interference pattern produced in the well-known double-slit experiment, thus obviating the quantum-mechanical notion of wave-particle duality.

In electrostatics, the precise empirical value of Coulomb's constant has been derived on purely theoretical grounds, while predictions have been derived of the hitherto unknown values of the electric dipole moments of electrons and protons. In thermodynamics, absolute zero temperature has been shown explicitly to be a prohibited state for any physical system, while in optics it has been shown that the refractive index of a medium and the associated physical effects (such as diffraction) depend fundamentally on the spatial geometry of the medium.

The framework of the proposed model is distinguished from the conventional framework primarily by its postulates pertaining to four essential features of nature, namely (i) the origin of energy and mass, (ii) the ontology of space and its physical structure, (iii) the character of time and its progression and (iv) the generative source of dynamic activity in nature. Because the proposed model defines each of these features with exactitude in terms that are rooted firmly in objective realism, it obviates the paradigm of abstract realities embedded in the descriptions of physical phenomena at both atomic and macroscopic scales.

It has been shown rigorously that the model presented in this paper has the capacity to not only reproduce and preserve the predictive power of conventional physical 
theories but to also enhance it by producing new predictions while resolving ambiguities inherent in the conventional theories. Given further that the proposed model represents a logically unified theoretical construct, it can be argued plausibly that this model offers a potentially viable alternative to contemporary models of theoretical physics.

\section{ACKNOWLEDGMENTS}

I thank Michael Astor and Darin Ragozzine for providing helpful comments, and Boris Sidorenko for implementing the computer simulation of the model presented in this report.

[1] M. Planck, The genesis and present state of development of the quantum theory (Jun 1920) in Nobel lectures, Physics 1901-1921 (Elsevier Publishing Co., Amsterdam, 1967).

[2] F. Berto and J. Tagliabue, Cellular automata (2012) in The Stanford encyclopedia of philosophy, edited by E. N. Zalta, https://plato.stanford.edu/entries/cellular-automata/.

[3] S. M. Ulam, On some mathematical problems connected with patterns of growth of figures, Proc. Symp. Appl. Math. 14, 215 (1962).

[4] J. von Neumann, Theory of self-reproducing automata, edited by A Burks (University of Illinois Press, Urbana, IL, 1966).

[5] M. Minsky, Cellular vacuum, Int. J. Theor. Phys. 21, 537 (1982).

[6] M. Gardner, Mathematical games: the fantastic combinations of John Conway's new solitaire game "life," Sci. Am. 223, 120 (1970).

[7] S. Wolfram, A new kind of science (Wolfram Media, Inc., Champaign, IL, 2002), pp. 433-546; pp. 1017-1065.

[8] G. 't Hooft, The cellular automaton interpretation of quantum mechanics (Springer, Berlin-Heidelberg-New York, 2016).

[9] J. A. Wheeler, Information, physics, quantum: the search for links in Complexity, entropy, and the physics of information (a proceedings volume in the Santa Fe Institute Studies in the Sciences of Complexity), edited by W. H. Zurek (Westview Press, Santa Fe, CA, 1990), pp. 309-336.

[10] N. Bohr, The quantum postulate and the recent development of atomic theory, Nature 121, 580 (1928).

[11] J. Hilgevoord and J. Uffink. The uncertainty principle (2016) in The Stanford encyclopedia of philosophy, edited by E. N. Zalta, https://plato.stanford.edu/entries/qt-uncertainty/.

[12] W. Pauli, Exclusion principle and quantum mechanics (Dec 1946) in Nobel lectures, Physics 1942-1962 (Elsevier Publishing Co., Amsterdam, 1964).

[13] E. Schrödinger, Quantization as an eigenvalue problem. (First Communication), Ann. Phys. (Berl.) 384, 361 (1926).

[14] E. Schrödinger, Quantization as an eigenvalue problem. (Second Communication), Ann. Phys. (Berl.) 384, 489 (1926).

[15] E. Schrödinger, Quantization as an eigenvalue problem. (Third Communication), Ann. Phys. (Berl.) 385, 437 (1926).

[16] E. Schrödinger, Quantization as an eigenvalue problem. (Fourth Communication), Ann. Phys. (Berl.) 386, 109 (1926).

[17] N. Bohr, The structure of the atom (Dec 1922) in Nobel lectures Physics 1922-1941 (Elsevier Publishing Co., Amsterdam, 1967).

[18] H. G. Dosch, Beyond the nanoworld: quarks, leptons and gauge bosons (A. K. Peters Ltd., Wellesley, MA, 2008), pp. 165-238.
[19] B. Moskowitz, The search for dark matter in the laboratory, New Sci. 122, 1660 (1989).

[20] L. Randall, What is dark matter? Nature 557, S6-S7 (2018).

[21] L. Amendola and S. Tsujikawa, Dark energy: theory and observations (Cambridge University Press, Cambridge, 2010).

[22] See Supplemental Material at https://osf.io/fcx8t/files to download (i) an executable computer simulation of the model presented in this report and the associated user guide (README.pdf), and (ii) a brief video demonstration of the computer simulation.

[23] Source: CODATA Task Group on Fundamental Constants (National Institute of Standards and Technology, Maryland, USA, 2015).

[24] A. Einstein, On a heuristic point of view concerning the production and transformation of light, Ann. Phys. (Berl.) 17. 132 (1905).

[25] A. Einstein, On the electrodynamics of moving bodies, Ann. Phys. (Berl.) 17. 891 (1905).

[26] A. Einstein, Does the inertia of a body depend on its energy content? Ann. Phys. (Berl.) 18. 639 (1905).

[27] M. Aigner and G. M. Ziegler, Proofs from the book (Springer-Verlag, Berlin, 2004), pp. 93-107.

[28] M. Schroeder, Fractals, chaos, power laws (W. H. Freeman \& Co., New York, 1991)

[29] A. H. Compton, A quantum theory of the scattering of X-rays by light elements, Phys. Rev. 21, 483 (1923).

[30] A. Einstein, On the movement of small particles suspended in stationary liquids required by the molecular-kinetic theory of heat, Ann. Phys. (Berl.) 17, 549 (1905).

[31] A. Einstein, On the theory of Brownian motion, Ann. Phys. (Berl.) 19, 371 (1906).

[32] R. P. Feynman, R. B. Leighton, and M. L. Sands, The Feynman lectures on physics (Addison-Wesley Press Co., Reading, MA, 1963), Vol. 1, Chap. 31; Vol. 2, Chap. 32-33.

[33] J. C. Maxwell, Theory of heat (Longman, Green \& Co., London, 1888).

[34] R. P. Feynman, R. B. Leighton, and M. L. Sands, The Feynman lectures on physics (Addison-Wesley Press Co., Reading, MA, 1963), Vol. 1, Chap. 39, 40 and 44.

[35] L. de Broglie, On the theory of quanta, Ann. Phys. (Paris) 3, 22 (1925).

[36] R. Bach, D. Pope, S-H Liou, and H. Batelaan, Controlled double-slit electron diffraction, New J. Phys. 15, 1 (2013).

[37] M. Longair, The new astrophysics in The new physics, edited by $\mathrm{P}$. Davies (Cambridge University Press, Cambridge, UK, 1989), pp. $160-169$.

[38] M. G. Kivelson and F. Bagenal, Planetary magnetospheres in Encyclopedia of the solar system, edited by P. R. Weissman et al. (Academic Press Limited, London,1999), pp. 477-497. 